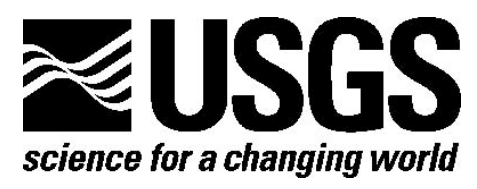

\title{
Hawaiian Volcano Observatory Seismic Data, January to March 2009
}

By Jennifer S. Nakata and Paul G. Okubo

Open-File Report 2010-1079

U.S. Department of the Interior

U.S. Geological Survey 


\section{U.S. Department of the Interior \\ KEN SALAZAR, Secretary}

\section{U.S. Geological Survey \\ Marcia K. McNutt, Director}

U.S. Geological Survey, Reston, Virginia: 2010

For product and ordering information:

World Wide Web: http://www.usgs.gov/pubprod

Telephone: 1-888-ASK-USGS

For more information on the USGS-the Federal source for science about the Earth, its natural and living resources, natural hazards, and the environment:

World Wide Web: http://www.usgs.gov

Telephone: 1-888-ASK-USGS

Suggested citation:

Nakata, J.S., and Okubo, P.G., 2010, Hawaiian Volcano Observatory seismic data, January to March 2009: U.S. Geological Survey Open-File Report 2010-1079, 47 p. [http://pubs.usgs.gov/of/2010/1079/].

Any use of trade, product, or firm names is for descriptive purposes only and does not imply endorsement by the U.S. Government.

Although this report is in the public domain, permission must be secured from the individual copyright owners to reproduce any copyrighted material contained within this report. 


\section{Contents}

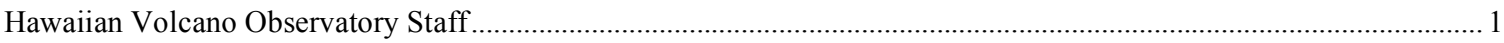

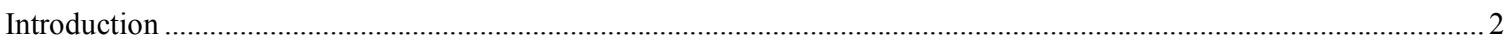

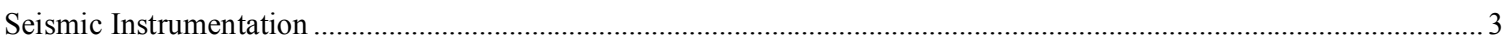

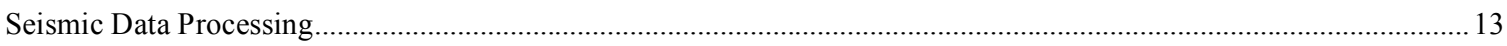

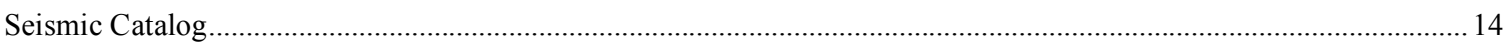

\section{Figures}

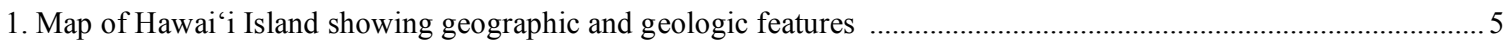

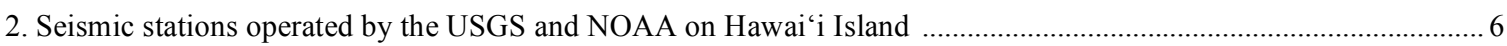

3. Seismic network telemetry scheme on Hawai 'i Island.............................................................................................

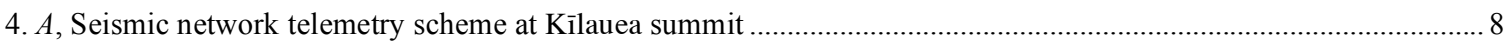

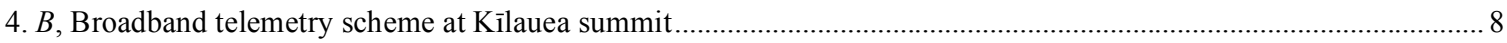

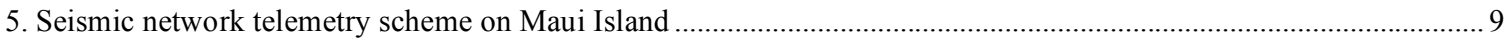

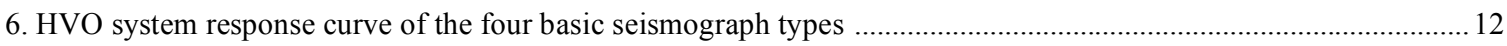

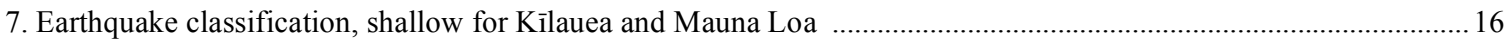

8. Earthquake classification, intermediate for Kỉlauea and Mauna Loa ..................................................................... 17

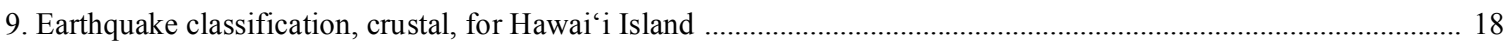

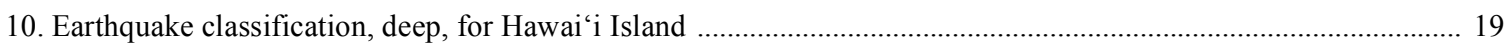

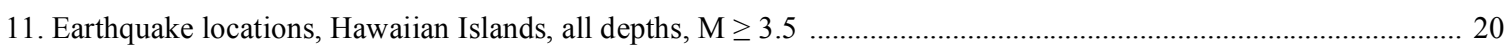

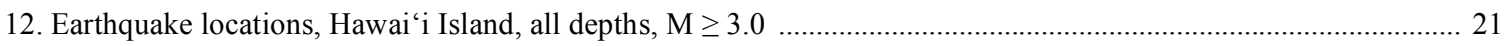

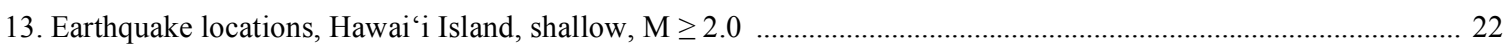

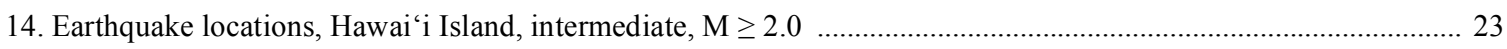

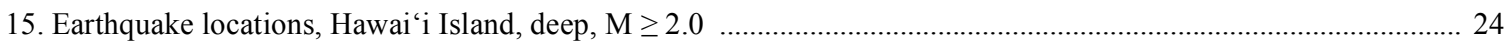

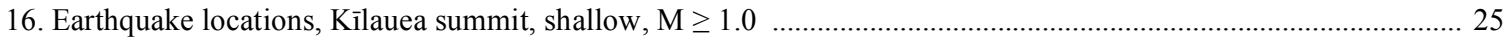

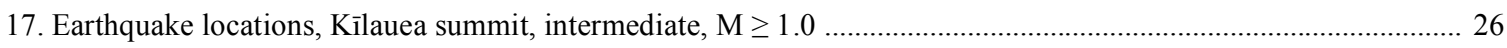

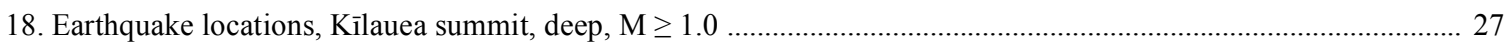

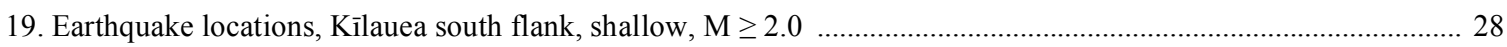

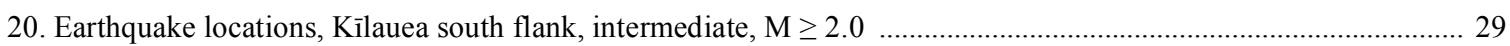

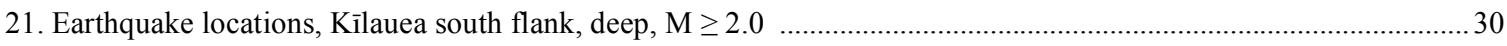

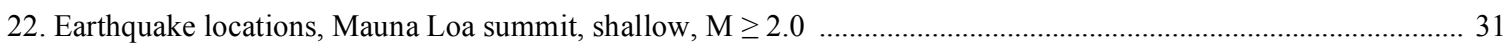

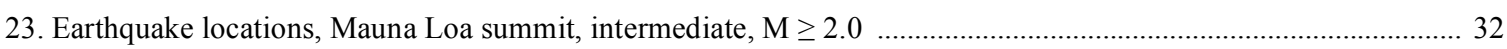

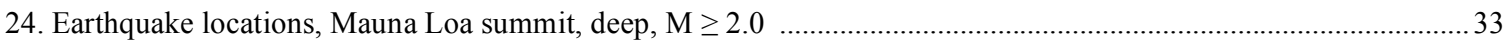

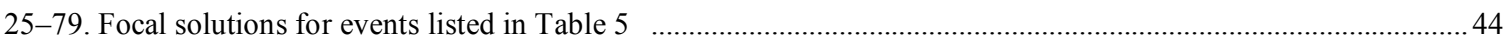

\section{Tables}

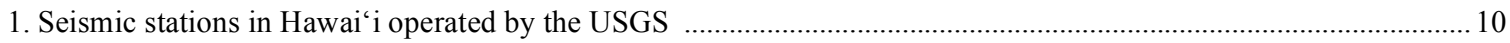

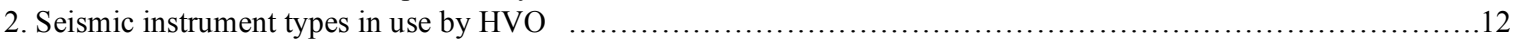

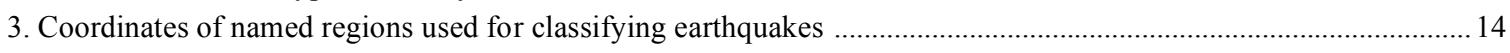

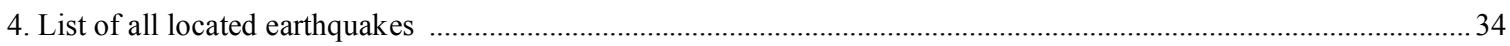

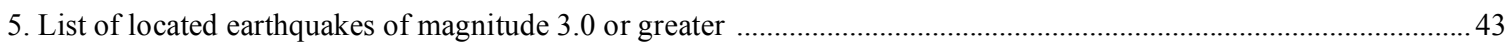




\title{
2009 HAWAIIAN VOLCANO OBSERVATORY STAFF
}

\author{
James P. Kauahikaua (Scientist-in-Charge)
}

Steve R. Brantley (Deputy Scientist-in-Charge)

\section{Geology}

Tim R. Orr

Matthew R. Patrick

Donald A. Swanson

Frank A. Trusdell

Kelly M. Wooten

\section{Geophysics}

James P. Kauahikaua

\section{Seismology}

Steven K. Fuke (Electronics)

Bruce T. Furukawa (Electronics)

Kenneth T. Honma (Electronics)

Jennifer S. Nakata

Paul G. Okubo

Jeff O. Uribe

David C. Wilson

\section{Deformation}

Kevan Kamabayashi

Asta Miklius

Michael P. Poland

Maurice K. Sako

Geochemistry

Tamar Elias

A. Jefferson Sutton

\section{Support}

Janet Babb (Media/Web Content/Outreach)

Robert Lopaka Lee (Computer)

T. Jane Takahashi (Library/Photo Archive)

\section{Administration}

Pauline N. Fukunaga (Webmaster)

Marian M. Kagimoto

Vicky Taylor+

Scientist Emeritus

C. Christina Heliker

Robert Y. Koyanagi

Arnold T. Okamura

\section{Post-Doctoral}

Takuji Yamada-Seismology

SCEP

David Trang

\section{Contracts}

Seismology:

L. Gladys Forbes (Record changer) Adolph R. Teves (Record changer)

\section{CSAV Cooperative Employees}

Loren Antolik (Deformation)

+Arrived in 2009. 


\section{Introduction}

This U.S. Geological Survey (USGS), Hawaiian Volcano Observatory (HVO) summary presents seismic data gathered during January-March 2009. The seismic summary offers earthquake hypocenters without interpretation as a source of preliminary data and is complete in that most data for events of $\mathrm{M} \geq 1.5$ are included. All latitude and longitude references in this report are stated in Old Hawaiian Datum.

The HVO summaries have been published in various forms since 1956. Summaries prior to 1974 were issued quarterly, but cost, convenience of preparation and distribution, and the large quantities of data necessitated an annual publication, beginning with Summary 74 for the year 1974. Since 2004, summaries have been identified simply by year, rather than by summary number.

Summaries originally issued as administrative reports were republished in 2007 as Open-File Reports. All the summaries since 1956 are available at http://pubs.usgs.gov/of/2007/1316-1345/ (last accessed 02/24/2010).

In January 1986, HVO adopted CUSP (ㅁalifornia Institute of Technology $\underline{\text { USGS }}$ Seismic Processing). Summary 86, available at http://pubs.er.usgs.gov/usgspubs/ofr/ofr92301 (last accessed 02/24/2010), includes a description of the seismic instrumentation, calibration, and processing used in recent years. The present summary includes background information about the seismic network to provide the end user with an understanding of the processing parameters and how the data were gathered.

Earthworm software, documentation available at http://folkworm.ceri.memphis.edu/ew-doc/ (last accessed 02/24/2010), was first installed at HVO in 1999 as part of an upgrade to tsunami warning capabilities in the Pacific region. This improved and expanded data exchange with the Pacific Tsunami Warning Center in Ewa Beach, Oahu, that included not only seismic waveforms, but also parametric earthquake data. Although Earthworm does included modules for earthquake triggering and earthquake location, this software was never used to generate catalog hypocenter locations at HVO.

During 2009, HVO migrated from CUSP to seismic processing software developed by the $\underline{\text { California }} \underline{\text { Integrated }} \underline{\text { Seismic }}$ $\underline{N}$ etwork or CISN. This software is now referred to as AQMS, for $\underline{A} d v a n c e d$ National Seismic System Quake $\underline{\text { Management }} \underline{\text { System. }}$ Summary data for this year will be presented in two reports; the first report includes earthquakes processed on the CUSP platform for January-March; earthquakes for the last three quarters, processed on the AQMS platform, will be published in a separate summary with a description of AQMS production parameters.

A report by Klein and Koyanagi (1980) ${ }^{1}$ tabulates instrumentation, calibration, and recording history of each seismic station in the network. It is designed as a reference for users of seismograms and phase data and includes and augments the information in the station table in this summary.

Figures 11-14 are maps showing computer-located hypocenters. The maps were generated using the Generic Mapping Tools (GMT), found at http://gmt.soest.hawaii.edu/ (last accessed 01/22/2010), in place of traditional QPLOT maps.

\footnotetext{
${ }^{1}$ Klein, F.W., and Koyanagi, R.Y., 1980, Hawaiian Volcano Observatory seismic network history, 1950-1979: U.S. Geological Survey Open-File Report 80-302, 84 p.
} 


\section{Seismic Instrumentation}

\section{The Network}

The Hawaiian Volcano Observatory maintains an extensive short-period (SP), telemetered seismic network on the Island of Hawai'i. Opportunities to augment the analog SP network according to specific monitoring or research interests have resulted in the installation and operation of a smaller number and focused deployments of digital broadband instruments. Data from the broadband instruments are not yet routinely used in catalog production, and the summary data are derived from the analog data streams.

The standard HVO SP field sensors, 1-Hz geophones, are deployed as single-component, vertical-only units or as threecomponent combinations of one vertical and two orthogonal horizontal units. The 2009 SP network consisted of 42 SP station sites: 10 three-component, 2 four-component (Uwēkahuna included a low-gain vertical with a unity-gain setting; 'Ainapō included a moderate-gain vertical with a 48-db setting), 2 two-component (each site included a moderate-gain vertical with a 48-db setting), and 28 vertical-component-only sites. The network coverage is most dense on and around Kîlauea Volcano. During 1999, HVO added to the network three vertical-component-only sites on the Island of Maui; these sites were not in operation during 2009. All seismic signals from the network are telemetered in real time to HVO for recording.

In 1994, initially viewed as a test deployment in collaboration with USGS, Menlo Park, California, stations equipped with USGS Digital Seismic Telemetry (DST) systems were installed to record volcanic seismic signals within Kilauea Volcano's summit caldera. A description by Dawson and others is available at http://pubs.er.usgs.gov/usgspubs/ofr/ofr98108 (last accessed 02/24/2010). These stations were first equipped with sensors of a few different types, but, over time, the stations have been standardized with Guralp CMG40T seismometers whose frequency responses have been extended to 50-second corner frequencies at their low-frequency operating range. Until the installation at HVO of Earthworm systems and subsequent modifications to the DST data acquisition footprint, the DST and analog data streams were recorded separately.

The National Ocean and Atmospheric Administration (NOAA), Pacific Tsunami Warning Center (PTWC), operates and maintains a network of SP stations on the islands of Hawai' $i$, Maui, and O'ahu. In 1999, radio links were established to share realtime data between PTWC and HVO. PTWC signals from one O'ahu three-component station and one Maui and five Hawai' $\mathrm{i}$ verticalcomponent-only stations were telemetered to HVO for recording. During 2009, telemetered signals were converted to real-time internet data streams between HVO and PTWC. In recent years, PTWC began replacing their SP sensors with broadband instruments.

Figure 1 is a map of selected geographic and geologic features. Figure 2 shows the sites of seismic stations operated by HVO and PTWC on the Island of Hawai'i during 2008. Figure 3 indicates the analog telemetry scheme for the seismic stations on Hawai' $\mathrm{i}$ Island using UHF or VHF frequencies, and figure 4 shows expanded views of the telemetry schemes at Kilauea summit- $4 A$, HVO seismic stations and $4 B$, a Kîlauea caldera broadband. Figure 5 indicates the telemetry scheme for the seismic stations on Maui Island that are no longer operational.

Table 1 lists SP seismic stations by site name, four-letter component codes, coordinates in degrees and minutes, (Old Hawaiian datum), elevation in meters, and other data, as described below, pertaining to each component. The list includes all SP station components operated by HVO during 2009 and reviewed in CUSP by the data analysts. All station names with field sensors installed at the site remained on the list, though operation may not have been continuous. SP seismic-station components operated by PTWC on the Islands of Hawai' $\mathrm{i}, \mathrm{O} \mathrm{O}^{`} \mathrm{ahu}$, and Maui are also listed. Phase readings from PTWC stations that are not telemetered to HVO are used to supplement data for local earthquakes and earthquakes that occur within the Hawaiian Archipelago but are distant from the Hawai‘i Island network. No earthquakes during this report period required additional PTWC phase readings.

\section{Instrumentation and Recording}

Each telemetered SP station's sensor is connected to a voltage-controlled oscillator (VCO) for FM multiplex transmission to HVO by UHF or VHF radio. These telemetering stations are all of Type 1, Earthquake Hazards Team (EHT) standard system used in USGS seismic networks (see table 2 for details). After discrimination at the receiver, signals pass through an analog-to-digital converter as part of the routine computer location processing and archiving. Through July 2001, continuous signals from the telemetered network were saved on 4-mm digital-audio tape (DAT) recording units. Three DAT recorders ran in automatic rotation as each $\sim 20$-hr tape was filled. Optic recordings are coded in table 1 as follows: H, Helicorder paper, and I, ink paper. DAT and paper records are archived at $\mathrm{HVO}$.

Beginning in April 2007, continuous signals digital records have been archived locally, as SAC files, to removable hard drives, though they were not accessible for viewing on the CUSP timing platform. 


\section{Seismograph Response and Calibration}

The response curve for the short-period seismograph type in use is given in figure 6. The Type 1 curve gives the magnification of the standard EHT system from ground motion at the seismometer to the seismic trace, as would be seen on a 20x Develocorder film viewer. The curve plots the unit response, which is multiplied by CAL, a station's calibration factor, to get the response for that station. Individual CAL factors for Type 1 seismographs are Develocorder-equivalent peak-to-peak amplitudes, measured in millimeters, of a 100-microvolt 5- to 8-Hz signal introduced to the preamp/VCO in place of the geophone at the field station. The calibration process normally is performed each time a station is visited for other required maintenance. Though Develocorder operations have ceased, calculations continue to be based on Develocorder equivalents. 


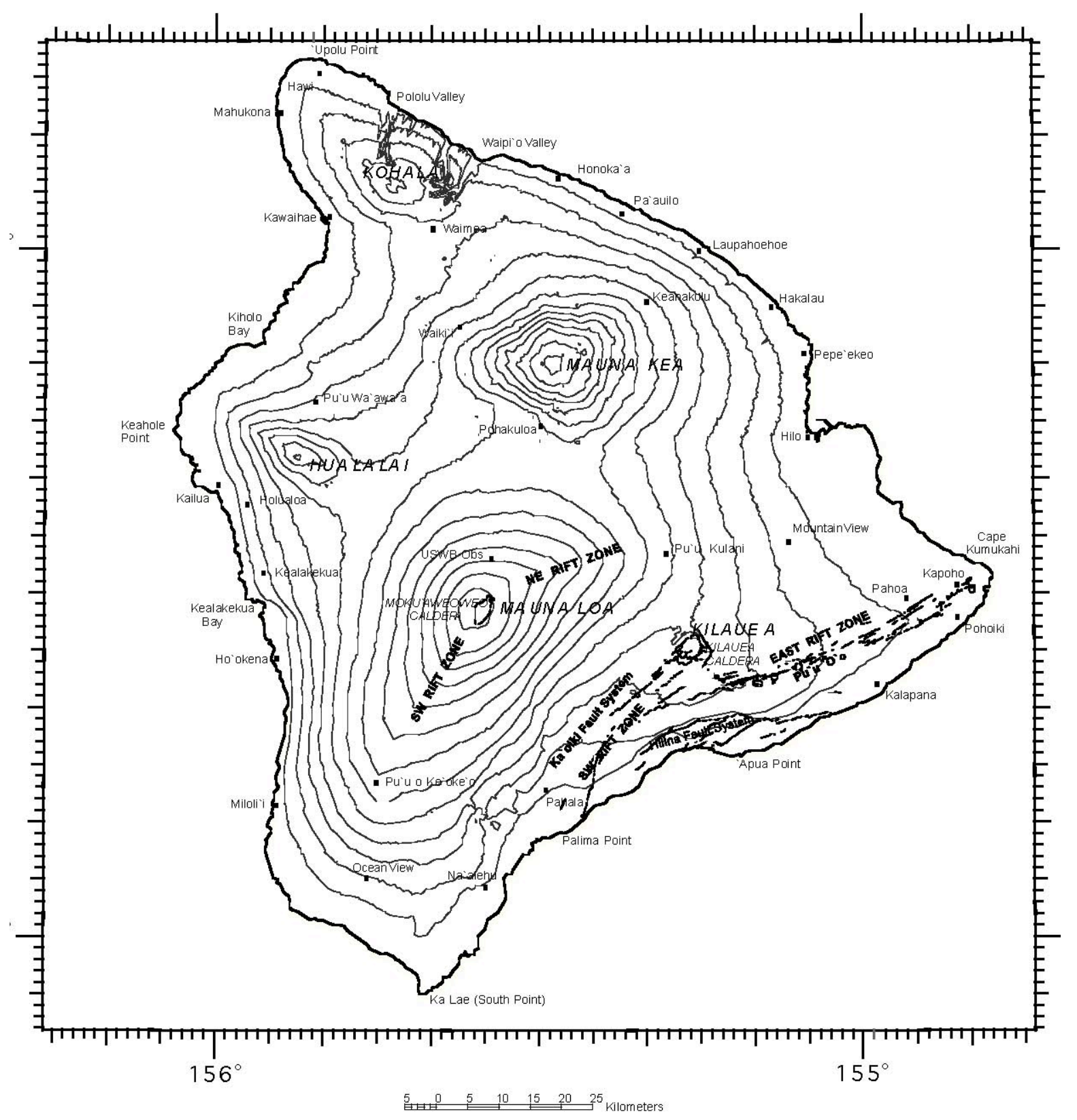

Figure 1. Map of Hawai'i Island showing principal settlements and other selected geologic features. The contours are in 1,000foot intervals. 


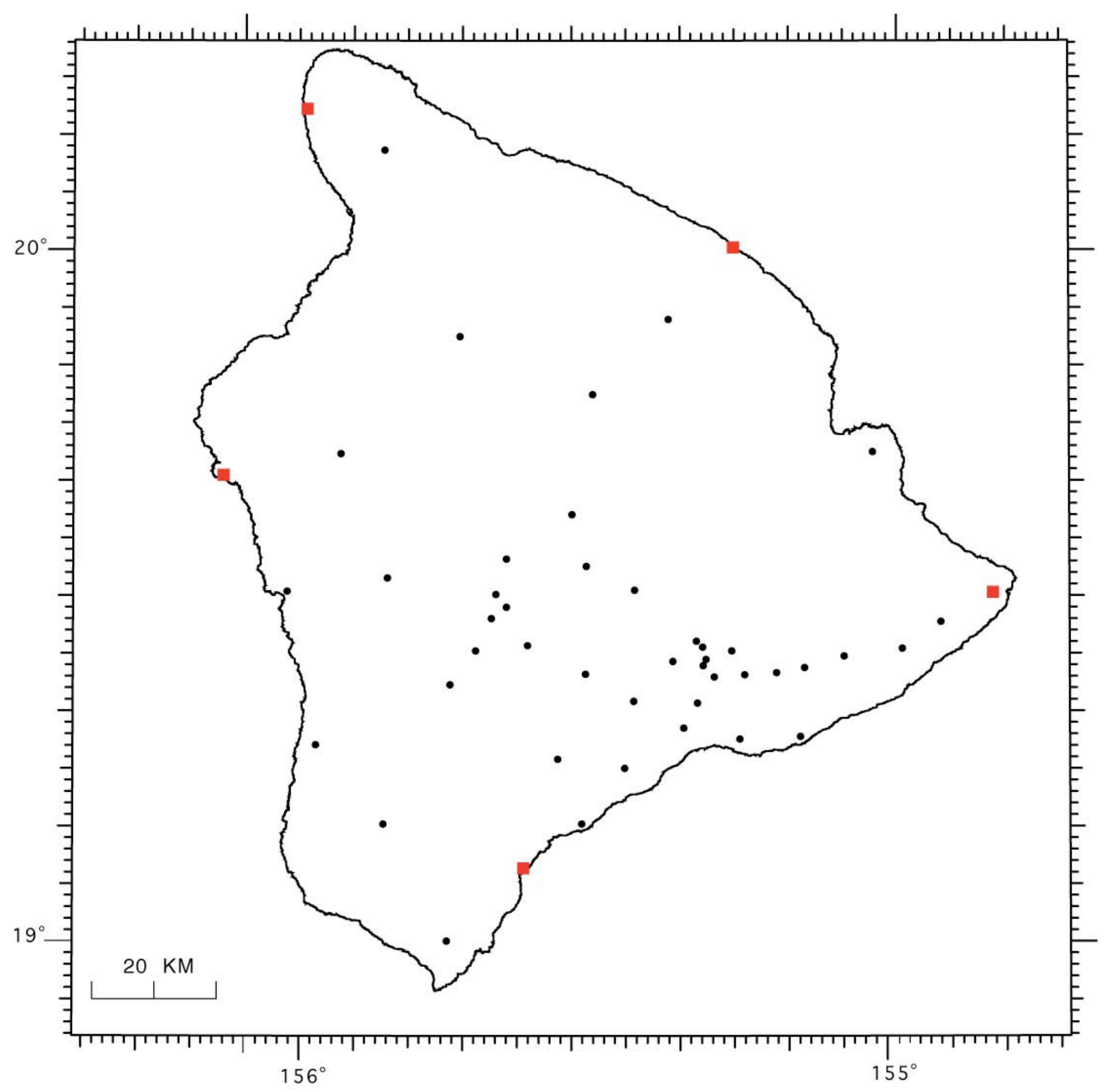

- Hawaiian Volcano Observatory network site

Pacific Tsunami Warning Center station site

Figure 2. The 2009 Hawaiian Volcano Observatory and Pacific Tsunami Warning Center seismic network on Hawai‘i Island. 


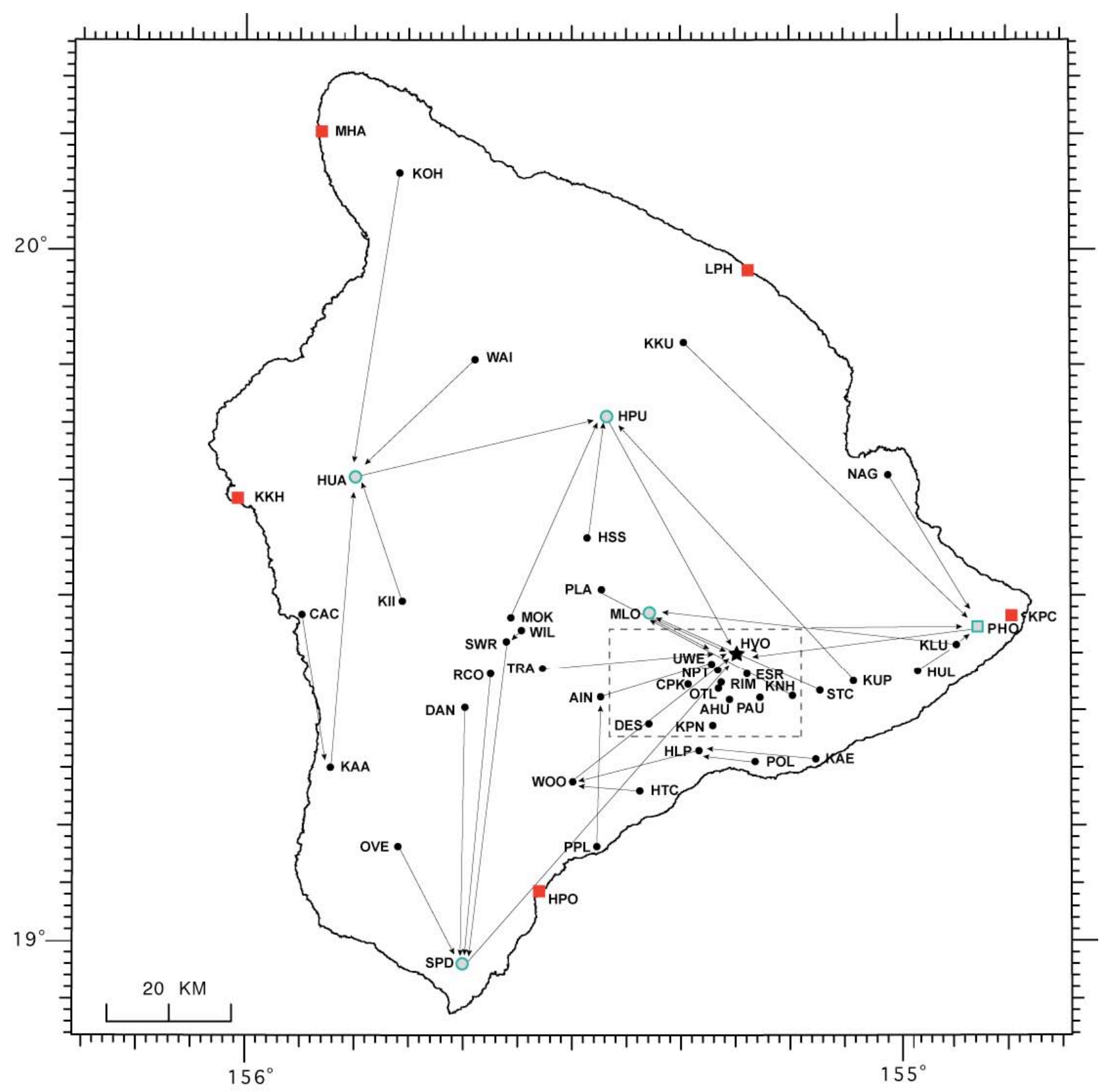

$\star \quad$ Hawaiian Volcano Observatory

- Hawaiian Volcano Observatory network site

- Direct-to-Line 32 Channel

$\square \quad$ Direct-to-Line 32 Channel repeater site

Kilauea Summit inset expanded in figure 4

n Pacific Tsunami Warning Center station site

Figure 3. Telemetry scheme for the 2009 Hawaiian Volcano Observatory and Pacific Tsunami Warning Center seismic network on Hawai'i Island. Figure 4 is an expanded view of Kilauea summit, indicated by the broken line. 

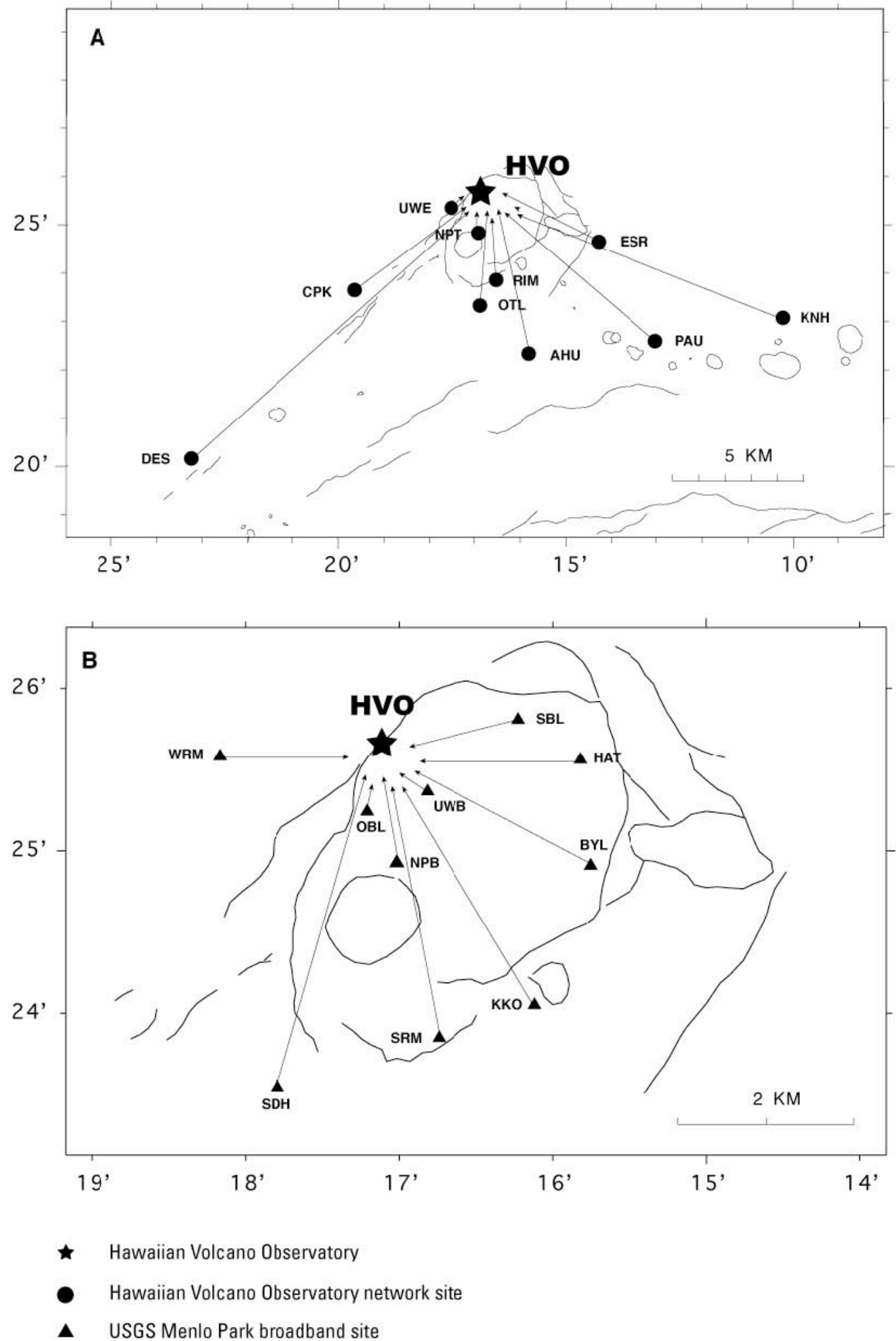

Figure 4. Expanded view of the Kỉlauea summit inset in figure 3 showing the telemetry scheme for $A$, the 2009 Hawaiian Volcano Observatory seismic network sites and $B$, the 2009 U.S. Geological Survey Menlo Park broadband seismic network on Hawai'i Island. 


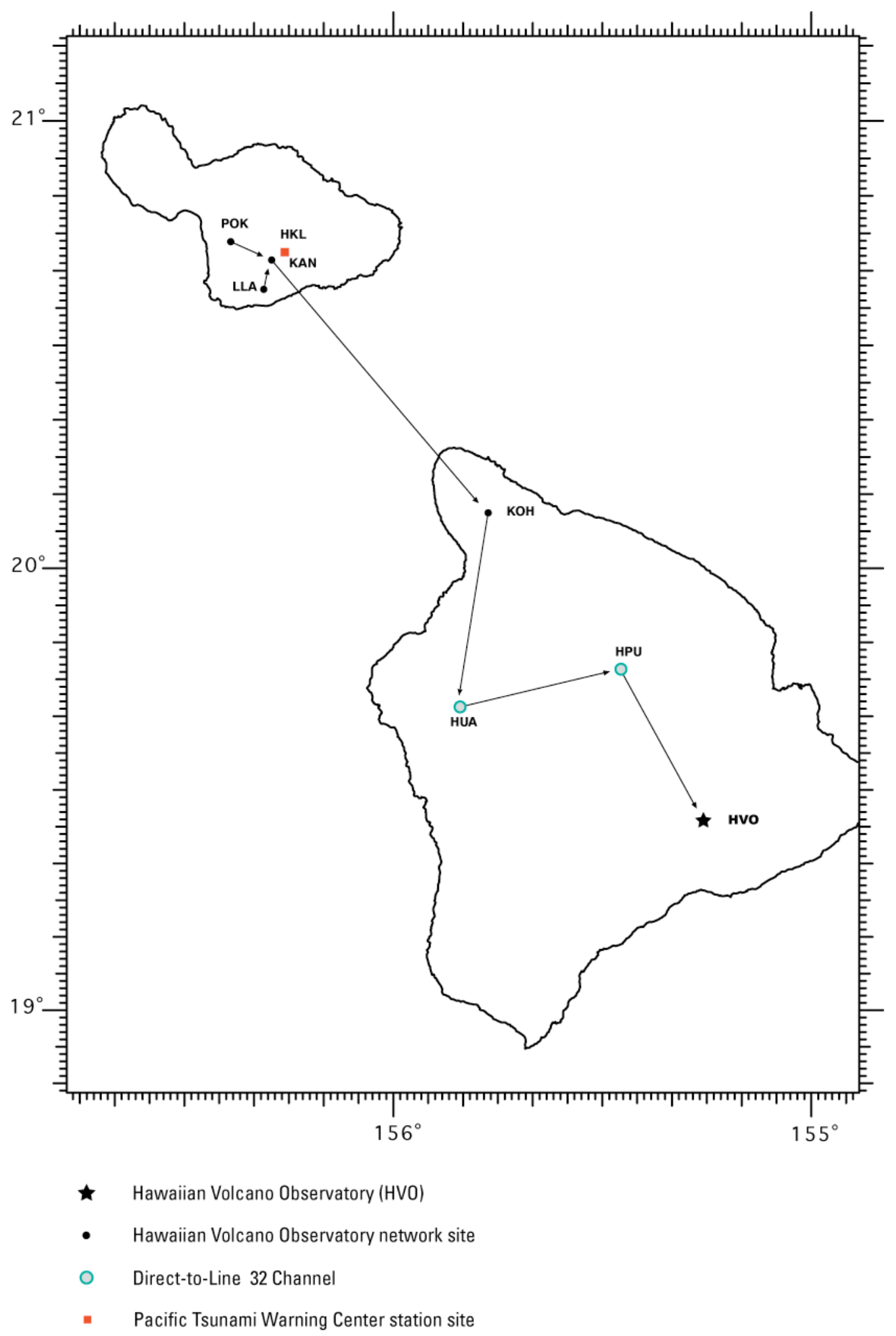

Figure 5. Telemetry scheme for the 2009 Hawaiian Volcano Observatory and Pacific Tsunami Warning Center seismic network on Maui Island, Hawai'i. The HVO stations were not in operation and, thus, produced no phase data for the January-March 2009 catalog. 
Table 1. Short-period seismic-station sites and components operated by the U.S. Geological Survey on Hawai'i Island during 2009.

[During the year, there may have been outage periods that required station maintenance at certain sites.]

\begin{tabular}{|c|c|c|c|c|c|c|c|c|c|c|c|c|}
\hline \multirow[t]{2}{*}{ STATION NAME } & \multirow[t]{2}{*}{ CODE } & \multicolumn{2}{|c|}{--LAT--- } & \multicolumn{2}{|c|}{$---\mathrm{LON}---$} & \multirow{2}{*}{$\begin{array}{l}\text { ELEV } \\
(M)\end{array}$} & DELAY & \multirow{2}{*}{$\begin{array}{c}\text { DELAY } \\
2\end{array}$} & \multirow{2}{*}{ CAL } & \multicolumn{3}{|c|}{ SEIS OPTIC } \\
\hline & & & $\mathrm{M}$ & D & $\mathrm{M}$ & & 1 & & & & & \\
\hline AHUA & AHUV & 19 & 22.40 & 155 & 15.90 & 1070 & -0.10 & -0.13 & 2.6 & L5 & & I \\
\hline AHUA & AHUE & 19 & 22.40 & 155 & 15.90 & 1070 & -0.10 & -0.13 & 3.0 & E5 & MW & \\
\hline AHUA & AHUN & 19 & 22.40 & 155 & 15.90 & 1070 & -0.10 & -0.13 & 3.0 & E5 & MW & \\
\hline AINAPO & AINV & 19 & 22.50 & 155 & 27.62 & 1524 & 0.13 & 0.17 & 6.8 & L5 & & \\
\hline AINAPO & AINE & 19 & 22.50 & 155 & 27.62 & 1524 & 0.13 & 0.17 & 3.0 & L5 & MW & \\
\hline AINAPO & AINN & 19 & 22.50 & 155 & 27.62 & 1524 & 0.13 & 0.17 & 3.0 & L5 & MW & \\
\hline AINAPO & AINZ & 19 & 22.50 & 155 & 27.62 & 1524 & 0.13 & 0.17 & 0.0 & L5 & & \\
\hline CAPTAIN COOK & $\mathrm{CACV}$ & 19 & 29.29 & 155 & 55.09 & 323 & 0.00 & -0.16 & 1.1 & L5 & & \\
\hline CONE PEAK & CPKV & 19 & 23.70 & 155 & 19.70 & 1038 & -0.26 & -0.07 & 6.0 & L5 & & \\
\hline DANDELION & DANV & 19 & 21.42 & 155 & 40.04 & 3003 & -0.27 & 0.03 & 4.3 & E5 & & \\
\hline DESERT & DESV & 19 & 20.20 & 155 & 23.30 & 815 & -0.29 & -0.13 & 4.5 & L5 & & I \\
\hline DIAMOND HEAD, & OADHHZ & 21 & 16.12 & 157 & 48.25 & 137 & 0.00 & 0.00 & 0.0 & $\mathrm{~S} 13$ & & \\
\hline ESCAPE ROAD & ESRV & 19 & 24.68 & 155 & 14.33 & 1177 & -0.17 & -0.19 & 1.2 & L5 & & \\
\hline HALEAKALA, MAUI & I $\mathrm{HKLZ}$ & 20 & 42.63 & 156 & 15.55 & 3051 & 0.00 & 0.00 & 0.0 & S13 & & \\
\hline HILINA PALI & HLPV & 19 & 17.96 & 155 & 18.63 & 707 & 0.02 & 0.07 & 2.1 & L5 & & \\
\hline HONOLULU, OAHU & HONZ & 21 & 19.30 & 158 & 0.50 & 2 & 0.00 & 0.00 & 0.0 & S13 & & \\
\hline HONOLULU, OAHU & HONE & 21 & 19.30 & 158 & 0.50 & 2 & 0.00 & 0.00 & 0.0 & S13 & & \\
\hline HONOLULU, OAHU & HONN & 21 & 19.30 & 158 & 0.50 & 2 & 0.00 & 0.00 & 0.0 & S13 & & \\
\hline HONUAPO & $\mathrm{HPOZ}$ & 19 & 5.34 & 155 & 33.23 & 15 & 0.00 & 0.00 & 0.0 & $\mathrm{~S} 13$ & & \\
\hline HALE POHAKU & HPUV & 19 & 46.72 & 155 & 27.54 & 3396 & 0.31 & 0.17 & 3.3 & L5 & & \\
\hline HUMUULA SHEEP S & STHSSV & 19 & 36.31 & 155 & 29.13 & 2445 & 0.20 & 0.35 & 4.0 & L5 & & \\
\hline HUMUULA SHEEP S & STHSSE & 19 & 36.31 & 155 & 29.13 & 2445 & 0.20 & 0.35 & 3.0 & L5 & MW & \\
\hline HUMUULA SHEEP S & STHSSN & 19 & 36.31 & 155 & 29.13 & 2445 & 0.20 & 0.35 & 3.0 & L5 & MW & \\
\hline HOT CAVES & HTCV & 19 & 14.33 & 155 & 24.02 & 381 & -0.16 & -0.07 & 2.3 & $\mathrm{E} 4$ & & \\
\hline HUALALAI & HUAV & 19 & 41.25 & 155 & 50.32 & 2189 & 0.67 & 0.38 & 2.8 & L5 & & \\
\hline HEIHEIAHULU & HULV & 19 & 25.13 & 154 & 58.72 & 369 & -0.17 & -0.16 & 1.6 & L5 & & $\mathrm{H}$ \\
\hline HEI & HULE & 19 & 25.13 & 154 & 58.72 & 369 & -0.17 & -0.16 & 3.0 & E5 & MW & \\
\hline EIAHULU & HULN & 19 & 25.13 & 154 & 58.72 & 369 & -0.17 & -0.16 & 3.0 & L5 & MW & \\
\hline KAAPUNA & $\mathrm{KAAV}$ & 19 & 15.98 & 155 & 52.28 & 524 & -0.12 & -0.01 & 3.3 & E5 & & \\
\hline KAENA POINT & KAEV & 19 & 17.35 & 155 & 7.95 & 37 & -0.01 & 0.06 & 1.4 & L5 & & \\
\hline KANAHAU, MAUI & KANV & 20 & 41.60 & 156 & 17.84 & 2745 & 0.00 & 0.00 & 0.0 & L5 & & \\
\hline KANEKII & KIIV & 19 & 30.56 & 155 & 45.90 & 1841 & 0.15 & 0.37 & 3.0 & L5 & & \\
\hline KANEKII & KIIE & 19 & 30.56 & 155 & 45.90 & 1841 & 0.15 & 0.37 & 3.0 & L5 & MW & \\
\hline KANEKII & KIIN & 19 & 30.56 & 155 & 45.90 & 1841 & 0.15 & 0.37 & 3.0 & L5 & MW & \\
\hline KIPAPA, OAHU & KIPZ & 21 & 25.40 & 158 & 0.90 & 2 & 0.00 & 0.00 & 0.0 & S13 & & \\
\hline KAILUA, KONA & KKHZ & 19 & 39.40 & 156 & 1.12 & 1 & 0.00 & 0.00 & 0.0 & S13 & & \\
\hline KEANAKOLU & KKUV & 19 & 53.39 & 155 & 20.58 & 1863 & 0.68 & 0.24 & 3.3 & L5 & & \\
\hline PUU KALIU & KLUV & 19 & 27.48 & 154 & 55.26 & 271 & -0.17 & -0.30 & 3.4 & L5 & & \\
\hline KANE NUI O HAMO & $\mathrm{O} \mathrm{KNHV}$ & 19 & 22.95 & 155 & 10.32 & 954 & -0.17 & -0.20 & 0.0 & L5 & & I \\
\hline KANE NUI O HAMO & $\mathrm{O} \mathrm{KNHZ}$ & 1 & 22.95 & 155 & 10.32 & 954 & -0.17 & -0.20 & 0.0 & L5 & & \\
\hline KOHALA & KOHV & 20 & 7.69 & 155 & 46.77 & 1166 & -0.03 & -0.17 & 6.3 & L5 & & \\
\hline KOHALA & $\mathrm{KOHE}$ & 20 & 7.69 & 155 & 46.77 & 1166 & -0.03 & -0.17 & 3.0 & L5 & MW & \\
\hline KOHALA & KOHN & 20 & 7.69 & 155 & 46.77 & 1166 & -0.03 & -0.17 & 3.0 & L5 & MW & \\
\hline KAPOHO CONE & $\mathrm{KPCZ}$ & 19 & 30.02 & 154 & 50.51 & 134 & 0.00 & 0.00 & 0.0 & S13 & & \\
\hline KIPUKA NENE & KPNV & 19 & 20.10 & 155 & 17.40 & 924 & -0.11 & -0.08 & 3.5 & L5 & & \\
\hline KUPAINAHA & KUPV & 19 & 24.32 & 155 & 4.68 & 646 & -0.25 & -0.30 & 0.0 & L5 & & \\
\hline LUALAILUA, MAUI & I LLAV & 20 & 37.62 & 156 & 18.62 & 683 & 0.00 & 0.00 & 0.0 & L5 & & \\
\hline LAUPAHOEHOE & LPHZ & 19 & 59.82 & 155 & 14.58 & 1 & 0.00 & 0.00 & 0.0 & S13 & & \\
\hline MAHUKONA & MHAZ & 20 & 11.27 & 155 & 54.18 & 1 & 0.00 & 0.00 & 0.0 & $\mathrm{~S} 13$ & & \\
\hline MAUNA LOA & MLOV & 19 & 29.80 & 155 & 23.30 & 2010 & 0.03 & 0.08 & 5.6 & L5 & & I \\
\hline MAUNA LOA & MLOE & 19 & 29.80 & 155 & 23.30 & 2010 & 0.03 & 0.08 & 3.0 & L5 & MW & \\
\hline MAUNA LOA & MLON & 19 & 29.80 & 155 & 23.30 & 2010 & 0.03 & 0.08 & 3.0 & L5 & MW & \\
\hline MOKUAWEOWEO & MOKV & 19 & 29.28 & 155 & 35.98 & 4104 & 0.15 & 0.16 & 4.2 & L5 & & IH \\
\hline
\end{tabular}




\begin{tabular}{|c|c|c|c|c|c|c|c|c|c|c|c|c|}
\hline \multirow{2}{*}{ STATION NAME } & \multirow[t]{2}{*}{ CODE } & \multicolumn{2}{|c|}{--LAT- -- } & \multicolumn{2}{|c|}{$---\mathrm{LON}---$} & \multirow{2}{*}{$\begin{array}{l}\text { ELEV } \\
(M)\end{array}$} & DELAY & \multirow{2}{*}{$\begin{array}{c}\text { DELAY } \\
2\end{array}$} & \multirow{2}{*}{ CAL } & \multirow{2}{*}{\multicolumn{3}{|c|}{$\begin{array}{l}\text { SEIS OPTIC } \\
\text { TYPE RECORD }\end{array}$}} \\
\hline & & & $\quad M$ & D & M & & 1 & & & & & \\
\hline NATIONAL GUARD & NAGV & 19 & 42.12 & 155 & 1.72 & 18 & 0.54 & 0.30 & 4.0 & R5 & & \\
\hline NATIONAL GUARD & NAGE & 19 & 42.12 & 155 & 1.72 & 18 & 0.54 & 0.30 & 3.0 & R5 & MW & \\
\hline NATIONAL GUARD & NAGN & 19 & 42.12 & 155 & 1.72 & 18 & 0.54 & 0.30 & 3.0 & R5 & MW & \\
\hline NORTH PIT & NPTV & 19 & 24.90 & 155 & 17.00 & 1115 & -0.30 & -0.18 & 3.0 & L5 & & IH \\
\hline NORTH PIT & NPTE & 19 & 24.90 & 155 & 17.00 & 1115 & -0.30 & -0.18 & 3.0 & L5 & MW & \\
\hline NORTH PIT & NPTN & 19 & 24.90 & 155 & 17.00 & 1115 & -0.30 & -0.18 & 3.0 & L5 & MW & \\
\hline OPANA, OAHU & OPAZ & 21 & 41.45 & 158 & 0.70 & 100 & 0.00 & 0.00 & 0.0 & $\mathrm{~S} 13$ & & \\
\hline OUTLET & OTLV & 19 & 23.38 & 155 & 16.94 & 1038 & -0.19 & -0.18 & 2.6 & L5 & & \\
\hline OUTLET & OTLZ & 19 & 23.38 & 155 & 16.94 & 1038 & -0.19 & -0.18 & 0.0 & L5 & & \\
\hline OCEANVIEW ESTAT & EOVEV & 19 & 9.21 & 155 & 45.92 & 1378 & 0.00 & 0.00 & 0.0 & L5 & & \\
\hline PAUAHI & PAUV & 19 & 22.62 & 155 & 13.10 & 994 & -0.21 & -0.24 & 2.9 & L5 & & \\
\hline PAUAH I & PAUE & 19 & 22.62 & 155 & 13.10 & 994 & -0.21 & -0.24 & 3.0 & L5 & MW & \\
\hline PAUAH I & PAUN & 19 & 22.62 & 155 & 13.10 & 994 & -0.21 & -0.24 & 3.0 & L5 & MW & \\
\hline PUU ULAULA & PLAV & 19 & 32.00 & 155 & 27.67 & 2992 & -0.03 & 0.13 & 6.3 & L5 & & I \\
\hline PUUOKALI, MAUI & POKV & 20 & 44.00 & 156 & 23.32 & 511 & 0.00 & 0.00 & 0.0 & L5 & & \\
\hline POLIOKEAWE PALI & POLV & 19 & 17.02 & 155 & 13.47 & 169 & -0.02 & 0.03 & 3.4 & E5 & & \\
\hline PUU PILI & PPLV & 19 & 9.50 & 155 & 27.87 & 35 & -0.15 & -0.15 & 1.4 & E5 & & \\
\hline RED CONE & $\mathrm{RCOV}$ & 19 & 24.36 & 155 & 37.79 & 3601 & 0.00 & 0.00 & 0.0 & L5 & & \\
\hline RIM & RIMV & 19 & 23.90 & 155 & 16.60 & 1128 & -0.21 & -0.13 & 0.0 & L5 & & $\mathrm{H}$ \\
\hline RAINSHED & RSDV & 19 & 27.78 & 155 & 16.68 & 1270 & 0.06 & 0.15 & 0.0 & L5 & & \\
\hline SOUTH POINT & SPDV & 18 & 58.94 & 155 & 40.24 & 250 & -0.17 & -0.22 & 0.0 & L5 & & \\
\hline SOUTH POINT & SPDE & 18 & 58.94 & 155 & 40.24 & 250 & -0.17 & -0.22 & 0.0 & L5 & MW & \\
\hline SOUTH POINT & SPDN & 18 & 58.94 & 155 & 40.24 & 250 & -0.17 & -0.22 & 0.0 & L5 & MW & \\
\hline STEAM CRACKS & STCV & 19 & 23.30 & 155 & 7.67 & 765 & -0.25 & -0.30 & 3.4 & L5 & & $\mathrm{H}$ \\
\hline SOUTHWEST RIFT & SWRV & 19 & 27.26 & 155 & 36.30 & 4048 & 0.01 & 0.04 & 5.6 & E5 & & \\
\hline TRAIL & TRAV & 19 & 24.91 & 155 & 32.96 & 3207 & 0.00 & 0.00 & 0.0 & L5 & & \\
\hline UWEKAHUNA & URAV & 19 & 25.40 & 155 & 17.60 & 1240 & -0.21 & 0.00 & 0.0 & R5 & & \\
\hline UWEKAHUNA & URAE & 19 & 25.40 & 155 & 17.60 & 1240 & -0.21 & 0.00 & 3.0 & R5 & MW & \\
\hline UWEKAHUNA & URAN & 19 & 25.40 & 155 & 17.60 & 1240 & -0.21 & 0.00 & 3.0 & R5 & MW & \\
\hline UWEKAHUNA & UUGZ & 19 & 25.40 & 155 & 17.60 & 1240 & 0.00 & 0.00 & 0.0 & L0 & & \\
\hline WAIKII & WAIV & 19 & 51.58 & 155 & 39.60 & 1433 & 0.20 & 0.35 & 0.0 & L5 & & \\
\hline WILKES CAMP & WILV & 19 & 28.15 & 155 & 35.02 & 4037 & 0.22 & 0.17 & 2.6 & E5 & & \\
\hline WILKES CAMP & WILE & 19 & 28.15 & 155 & 35.02 & 4037 & 0.22 & 0.17 & 3.0 & L5 & MW & \\
\hline WILKES CAMP & WILN & 19 & 28.15 & 155 & 35.02 & 4037 & 0.22 & 0.17 & 3.0 & L5 & MW & \\
\hline WAIMANALO RIDGE & , WMRZ & 21 & 19.22 & 157 & 40.94 & 200 & 0.00 & 0.00 & 0.0 & $\mathrm{~S} 13$ & & \\
\hline WEATHER OBSERVA & TWOBV & 19 & 32.31 & 155 & 35.01 & 3396 & 0.00 & 0.00 & 0.0 & E5 & & \\
\hline WOOD VALLEY & WOOV & 19 & 15.08 & 155 & 30.12 & 909 & -0.15 & -0.06 & 2.6 & E5 & & \\
\hline
\end{tabular}


.Table 2 Seismic instrument types, U.S. Geological Survey, Hawaiian Volcano Observatory (HVO).

The codes in parentheses refer to the seismometer types listed in Table 1. Type 1 (Codes E, L, R, and 4, 5) consists of:

a) Geophone

Electrotech EV-17 (E), Mark Products L4C (L) or Kinemetric Ranger SS1 (R); (L) and (R) are 1.0-second period moving-magnet vertical- or horizontal- (E-W and N-S) component seismometers adjusted for an output of $0.5 \mathrm{volts} / \mathrm{cm} / \mathrm{sec}$ and 0.8 , critically damped.

b) Preamp/VCO USGS/OEVE Model J502, J512 (5) voltage-controlled oscillator. Three db points for bandpass filter at $0.1 \mathrm{~Hz}$ and $30 \mathrm{~Hz}$. Signals are transmitted on audio FM carrier over cable or FM-radio link to HVO.

Code (MW) Horizontal-component seismograph based on a Type 1 system and modified to 3x a Wood-Anderson response.

Code (S13) Geotech, 1-Hz seismometer with A1 VCO operated by the Pacific Tsunami Warning Center.

PERIOD, IN SECONDS

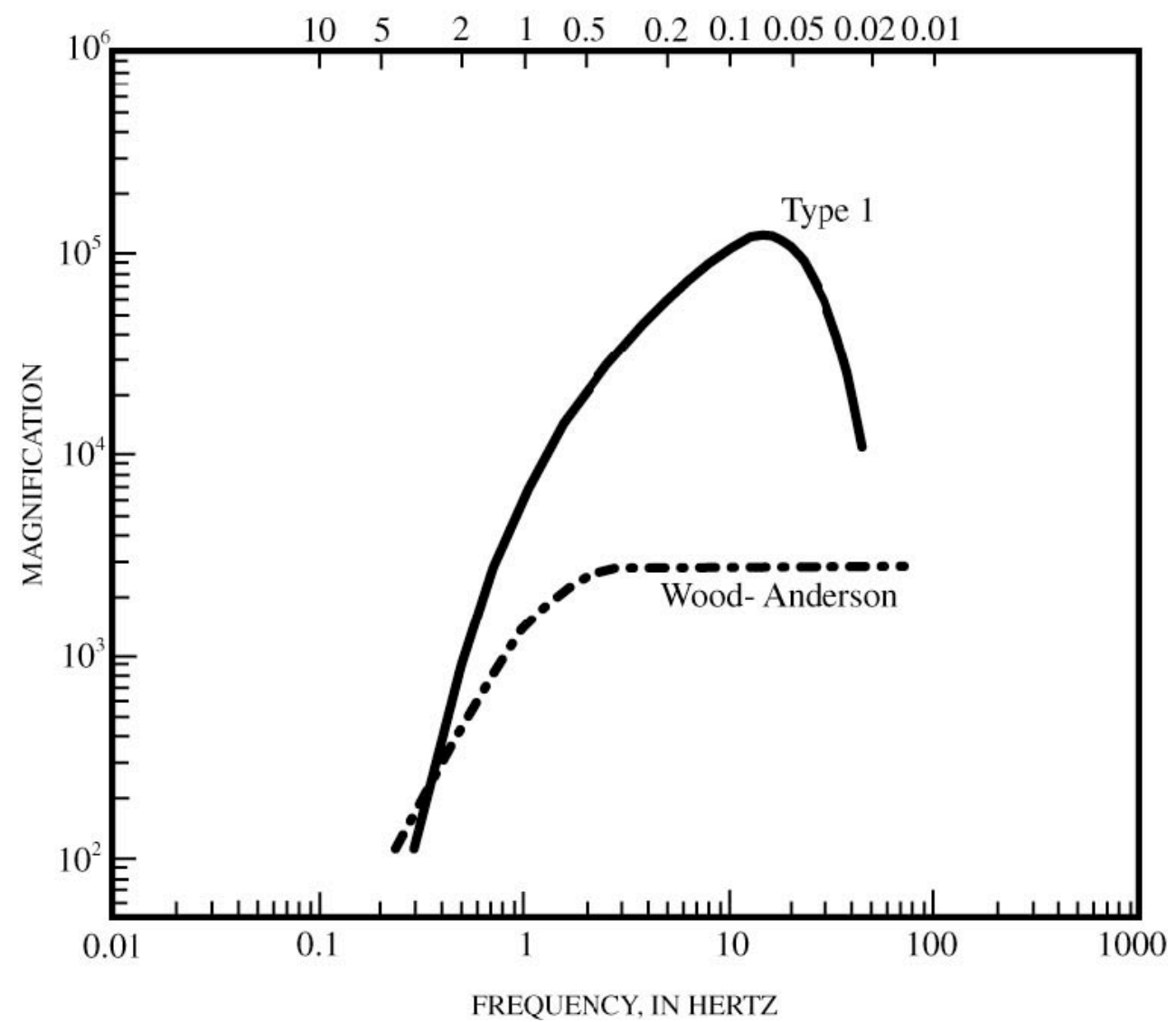

Figure 6. System-response curves for the Wood-Anderson torsion seismograph and for seismometers used by the Hawaiian Volcano Observatory. The Type 1 curve plots the unit response of the standard USGS microearthquake seismometer system as would be recorded on Develocorder film. This includes the geophone, all electronics, including telemetry, Develocorder galvanometer, and a 20x magnification film viewer. The curve plots the unit response, which is multiplied by CAL, a station's calibration factor, to get the response for that station. 


\section{Seismic Data Processing}

Due to age and high cost of maintenance, Develocorder "A" was discontinued on August 1, 1997. Daily count of classified micoearthquakes from source regions around Kỉlauea and Mauna Loa, and duration of tremor, were also discontinued. Coda duration, however, is measured in seconds from drum (ink or helicorder) records to determine a coda magnitude that is entered as an external magnitude in the final solution.

In 1986, HVO acquired a VAX 11-750 computer and adopted the CUSP routine. Discriminated signals pass through an analogto-digital converter, and detected events are saved in real time. Detected events are then demultiplexed, and P-picks are made by the computer, producing a rough location. Events are examined by an analyst on a graphics terminal in order to refine computer P-picks and to time additional P- and S-phases for a preliminary location. Binary .mem files are translated into ASCII phase files. Locations and amplitude magnitudes are then determined by using the program HYPOINVERSE-2000 (Klein, 2002) ${ }^{2}$. Events are reworked and rerun, as needed, to produce a final solution.

Through May 2005, CUSP .grm and .mem files were archived directly onto magneto-optical media. Since June 2005, the binary files have been archived to a second Alpha node, then stored on a RAID system as UNIX executable files. Files stored on the magneto-optical media through May 2005 were transferred to the RAID storage.

In July 1992, HVO acquired VAX servers and workstations needed to run the upgraded version of CUSP. The servers are used for data acquisition, and the workstations are used for interactive earthquake timing. In addition to timing $\mathrm{P}$ and $\mathrm{S}$ arrival signals, the VAX workstations were then capable of measuring peak-to-peak amplitudes, along with the associated period. This capability allowed the renewal of amplitude magnitude determinations from the network seismic stations. Amplitude data gathered from July 1992 to July 1997 became part of a test set to determine magnitude corrections for network stations. Results of newly determined magnitude corrections are detailed by Nakata and Okubo $(1997)^{3}$.

In parallel with CUSP, HVO currently operates Earthworm software to record all HVO seismic data, including the exchange of seismic data with cooperating networks. HVO also utilizes the Earthworm processing system for rapid computation of earthquake products (locations, magnitude, spectrograms, helicorders, ShakeMaps, and recent earthquakes Web pages). Analysis of triggered events and seismic-catalog generation was accomplished by using CUSP and HYPOINVERSE processing platforms through March 2009.

Earthquake hypocenters are computed within a one-dimensional velocity model. The model is specified by velocities at four depth points, as listed below. Velocity at any depth above a homogeneous half-space is given by linear interpolation between points:

\begin{tabular}{|c|c|}
\hline $\begin{array}{l}\text { VELOCITY } \\
(\mathrm{km} / \mathrm{sec})\end{array}$ & $\begin{array}{r}\text { DEPTH } \\
(\mathrm{km})\end{array}$ \\
\hline 1.9 & 0.0 \\
\hline 6.5 & 4.6 \\
\hline 6.9 & 15.0 \\
\hline 8.3 & $\geq 16.5$ \\
\hline
\end{tabular}

Two empirical sets of station delays or corrections are used in the HYPOINVERSE locations and are given in table 1. The delay models are separated by a circle of radius $34 \mathrm{~km}$, centered at $19^{\circ} 22^{\prime} \mathrm{N}$ and $155^{\circ} 10^{\prime} \mathrm{W}$. Delay model 1 is used for epicenters occurring within a circle of radius $31 \mathrm{~km}$ from the center. This region includes Kỉlauea and its south flank. A combination of both delay models is used for epicenters that fall in a transition zone that is $6 \mathrm{~km}$ wide. Delay model 2 is applied to the rest of the island and to offshore earthquakes. For a detailed description, refer to HYPOINVERSE-2000 (Klein, 2002) ${ }^{2}$.

Magnitudes for events are computed by using recorded amplitudes on selected network vertical, Modified Wood-Anderson (MW) horizontal, and/or moderate- and low-gain stations. Amplitude readings are corrected to an equivalent Wood-Anderson amplitude by using the curves shown in figure 6 and the CAL factors listed in table 1.

Duration magnitude is determined by the length of the signal, in seconds, read from drum recordings of Type 1 seismographs. This length of time is measured from the $\mathrm{P}$ arrival to the point where the earthquake signal has decayed to nearly the background noise level. Drum-recorded duration magnitude is calculated with a relationship equivalent to the develocorder viewer output.

\footnotetext{
${ }^{2}$ Klein, F.W., 2002' User's guide to HYPOINVERSE-2000, a Fortran Program to solve for earthquake locations and magnitudes: U.S.
} Geological Survey Open-File Report 02-171, 116 p.

${ }^{3}$ Nakata, J., and Okubo, P., 1997, Determination of station amplitude magnitude corrections for the Hawaiian Volcano Observatory telemetered seismograph network-Data from 1992-1997: U.S. Geological Survey Open-File Report 97-863, 73 p. 


\section{Seismic Catalog}

The emphasis of both station coverage and detailed data analysis is on the highly active southern half of the Island of Hawai' $\mathrm{i}$. The data set of well-recorded, located earthquakes in the Hawai' $i$ Island region is nearly complete above magnitude 2.0. Many smaller earthquakes in the Kilauea region are locatable because of the dense instrumental coverage. Substantial effort is made to locate earthquakes elsewhere within the Hawaiian Archipelago, and although such coverage cannot be as complete as it is in south Hawai'i, nearly all events above magnitude 4.0 are located with limited precision.

Data presented in the seismic catalog are in three parts: 1) Maps showing computer-located hypocenters are given in figures $11-$ 24. The location maps are of different scales and provide hypocenters with magnitude thresholds set at 1.0, 2.0, 3.0, and 3.5, varying according to region. 2) The list of computer locations constitutes the bulk of this summary and is given in table 4. Each earthquake in the list is assigned a three-letter code based on its general location and depth. Figures 7-10 are maps of the regions used to assign the location codes. The latitude and longitude limits of rectangular regions are listed in table 3 . When the listed coordinates overlap, boundaries are as shown in figures 7-10.3) Table 5 re-lists the events in table 4 for which the preferred magnitude is 3.0 or larger. This list includes many of the earthquakes felt in Hawai' $i$.

Table 3. Names and coordinates of regions used for classifying Hawaii earthquakes.

All earthquakes are HYPOINVERSE classified in one of the following groups, identified by a numerical class or three-letter code.

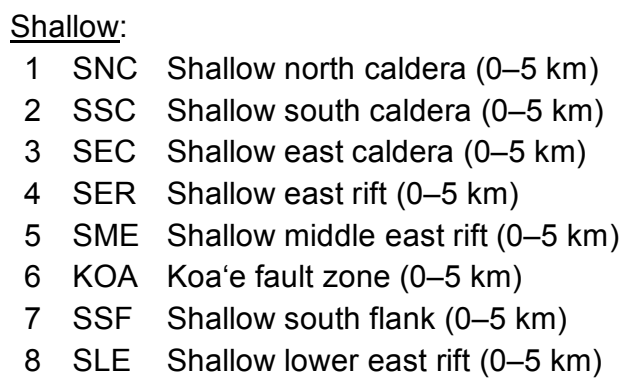

Intermediate depth:

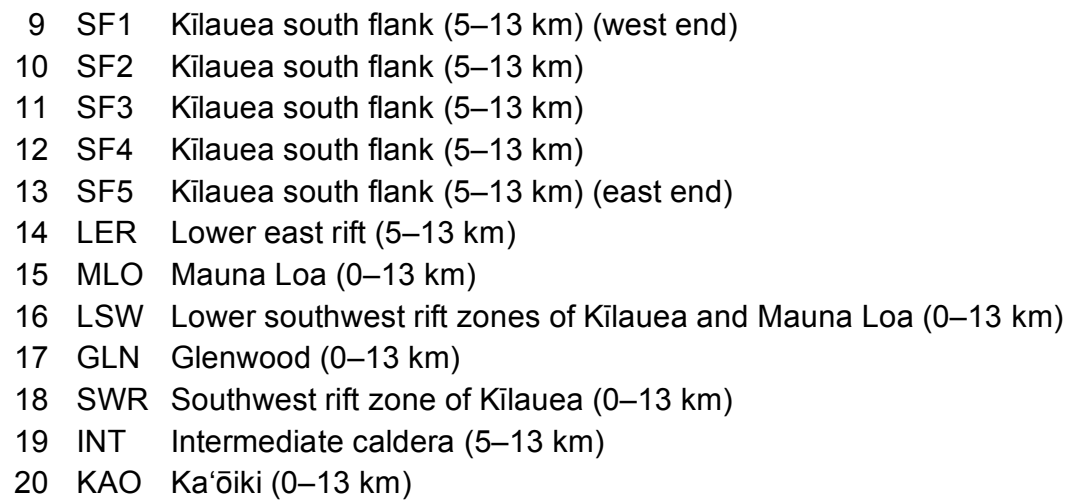

Deep:

21 DEP Deep Killauea (>13 km) (below regions 1-13, 17-19)

22 DLS Deep lower southwest rift zone of Kilauea and Mauna Loa (>13 km) (below region 16)

23 DML Deep Mauna Loa (>13 km) (below regions 15, 20)

Outer regions, all depths:

$\begin{array}{lll}24 & \text { LOI } & \text { Lō'ihi } \\ 25 & \text { KON } & \text { South Kona } \\ 26 & \text { HUA } & \text { Hualālai } \\ 27 & \text { KOH } & \text { Kohala } \\ 28 & \text { KEA } & \text { Mauna Kea } \\ 29 & \text { HIL } & \text { Hilo } \\ 30 & \text { DIS } & \text { Distant, everywhere else }\end{array}$


Table 3 (continued).

The latitude and longitude limits of the regions are given below. If the coordinates overlap, boundaries are as shown in figures 7-10.

\begin{tabular}{|c|c|c|c|c|c|}
\hline No. & Code & N. Lat. & S. Lat. & W. Lon. & E. Lon. \\
\hline 1 & SNC & 1928.0 & 1924.5 & 15519.0 & 15514.0 \\
\hline 2 & SSC & 1924.5 & 1922.0 & 15519.0 & 15516.5 \\
\hline 3 & SEC & 1924.5 & 1922.0 & 15516.5 & 15514.0 \\
\hline 4 & SER & 1926.0 & 1920.5 & 15514.0 & 15507.2 \\
\hline 5 & SME & 1926.0 & $1921.75-1920.0$ & 15507.2 & 15500.0 \\
\hline 6 & $\mathrm{KOA}$ & 1922.0 & 1920.5 & 15517.0 & 15514.0 \\
\hline 7 & SSF & $1920.6-1924.0$ & 1910.0 & 15517.0 & 15500.0 \\
\hline 8 & SLE & 1932.0 & 1916.0 & 15500.0 & 15440.0 \\
\hline 9 & SF1 & 1922.0 & 1910.0 & 15517.0 & 15514.5 \\
\hline 10 & SF2 & 1926.0 & 1910.0 & 15514.5 & 15512.3 \\
\hline 11 & SF3 & 1926.0 & 1910.0 & 15512.3 & 15509.1 \\
\hline 12 & SF4 & 1926.0 & 1910.0 & 15509.1 & 15505.3 \\
\hline 13 & SF5 & 1926.0 & 1910.0 & 15505.3 & 15500.0 \\
\hline 14 & LER & 1932.0 & 1916.0 & 15500.0 & 15440.0 \\
\hline 15 & MLO & 1935.0 & 1919.0 & 15535.0 & 15519.0 \\
\hline 16 & LSW & 1919.0 & 1840.0 & 15543.0 & 15525.0 \\
\hline 17 & GLN & 1935.0 & 1926.0 & 15519.0 & 15500.0 \\
\hline 18 & SWR & 1922.0 & 1910.0 & 15525.0 & 15517.0 \\
\hline 19 & INT & 1928.0 & 1922.0 & 15519.0 & 15514.0 \\
\hline 20 & KAO & 1930.0 & 1919.0 & 15532.0 & 15519.0 \\
\hline 21 & DEP & 1935.0 & 1910.0 & 15525.0 & 15500.0 \\
\hline 22 & DLS & 1919.0 & 1840.0 & 15543.0 & 15525.0 \\
\hline 23 & DML & 1935.0 & 1919.0 & 15535.0 & 15519.0 \\
\hline 24 & LOI & 1910.0 & 1840.0 & 15525.0 & 15500.0 \\
\hline 25 & $\mathrm{KON}$ & 1939.0 & 1900.0 & 15620.0 & 15543.0 \\
\hline 26 & HUA & 1955.0 & 1939.0 & 15620.0 & 15543.0 \\
\hline 27 & $\mathrm{KOH}$ & 2025.0 & 1955.0 & 15620.0 & 15534.0 \\
\hline 28 & KEA & 2025.0 & 1935.0 & 15534.0 & 15440.0 \\
\hline 29 & HIL & 1947.0 & 1932.0 & 15509.0 & 15440.0 \\
\hline
\end{tabular}




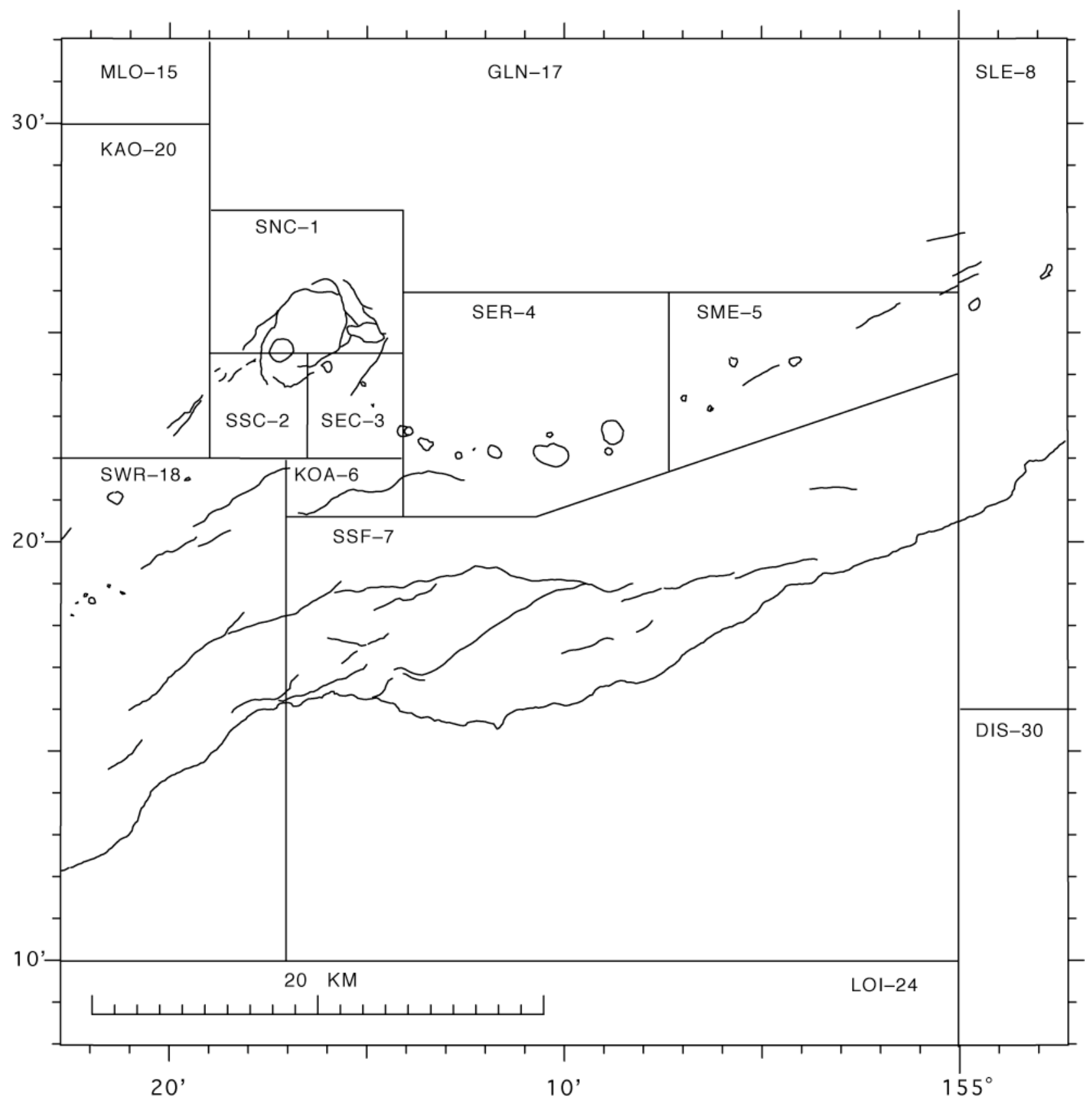

Figure 7. Regions for earthquake classification for Kïlauea and the east flank of Mauna Loa, Hawai'i. Depth range and coordinate boundaries for each region are described in table 3. 


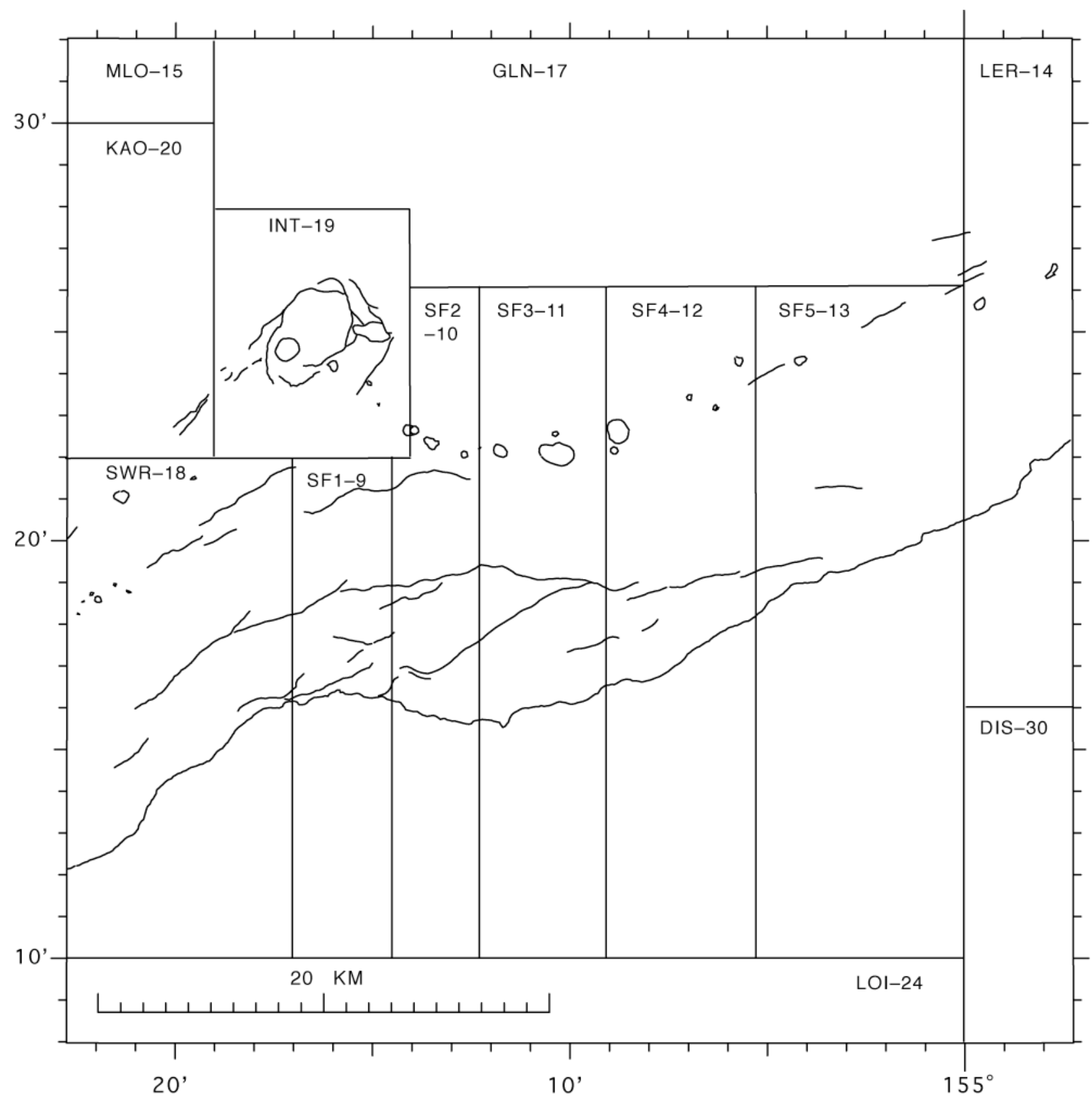

Figure 8. Regions for earthquake classification for Kïlauea and the east flank of Mauna Loa, Hawai'i. Depth range and coordinate boundaries for each region are described in table 3. 


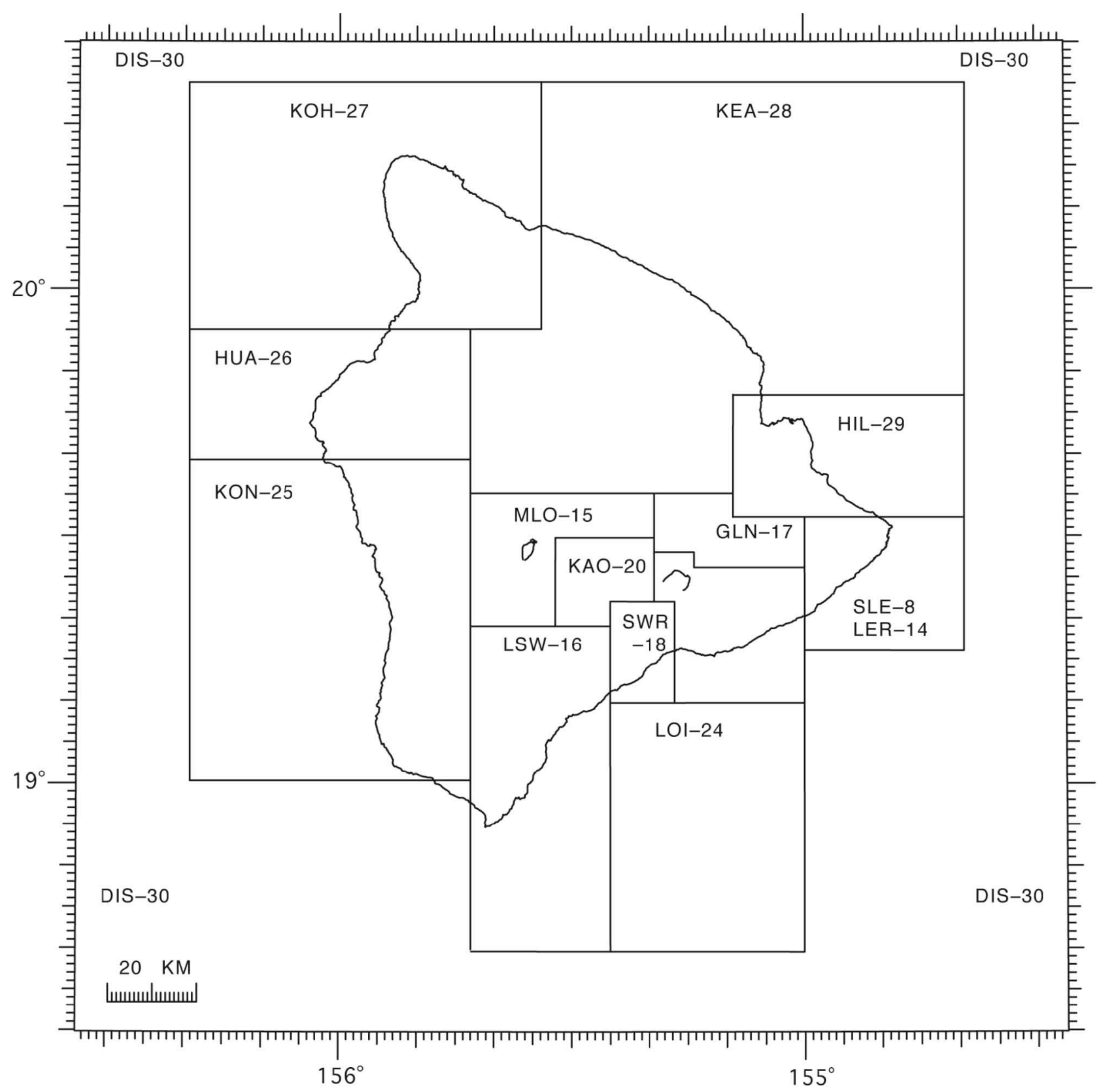

Figure 9. Regions for earthquake classification for Hawai'i Island. Depth range and coordinate boundaries for each region are described in table 3 . 


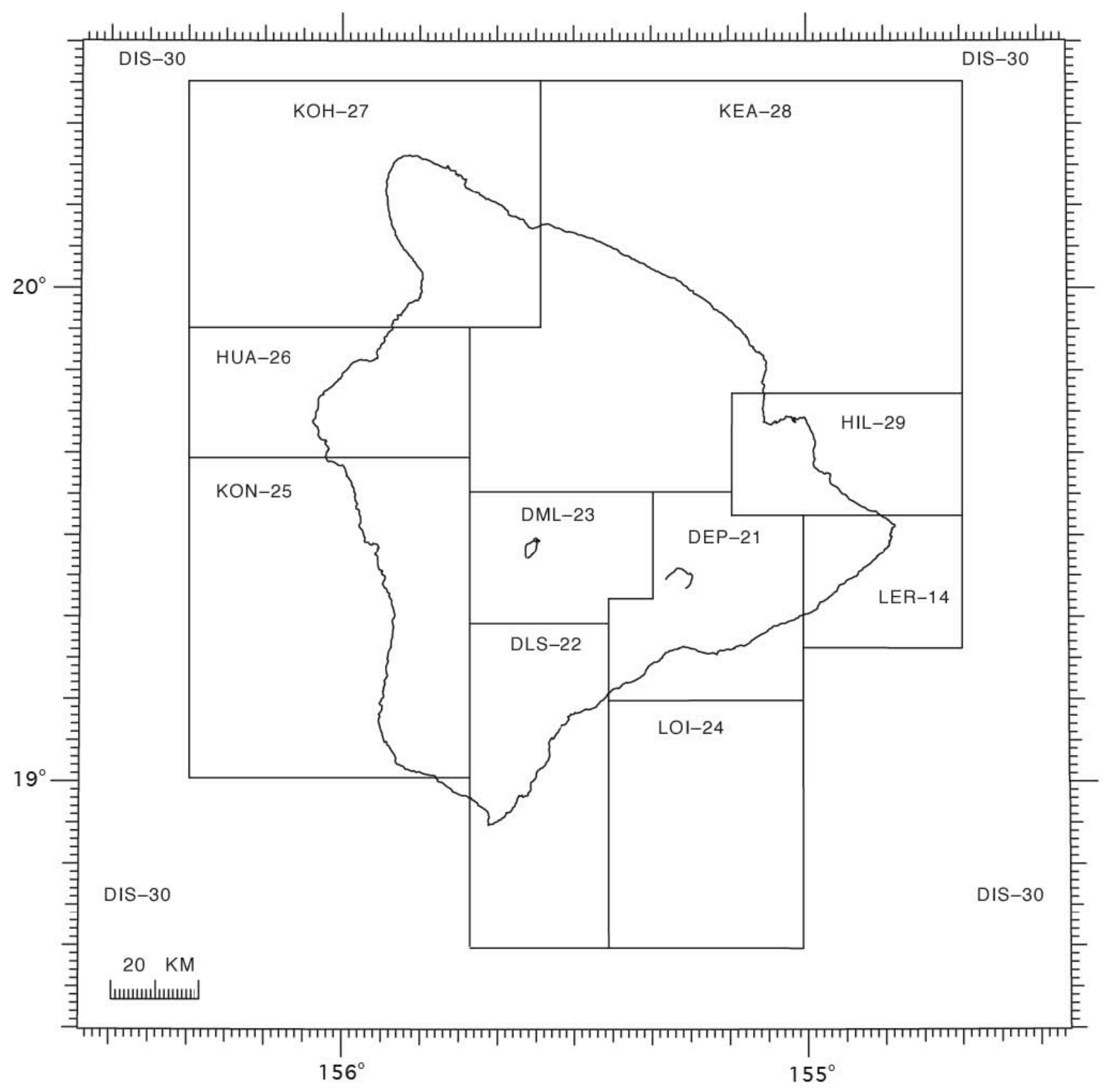

Figure 10. Regions for earthquake classification for Hawai'i Island. Depth range and coordinate boundaries for each region are described in table 3 . 


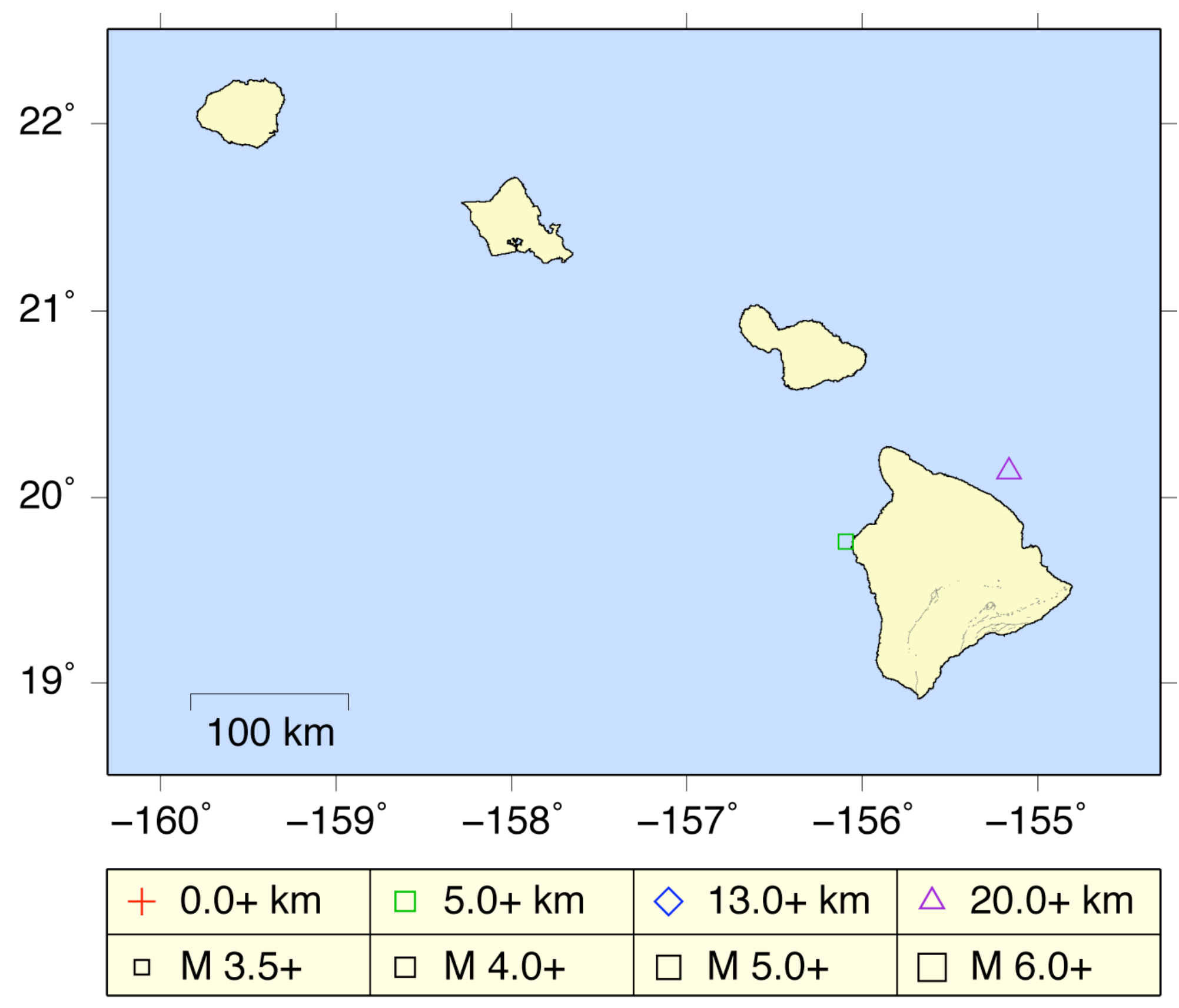




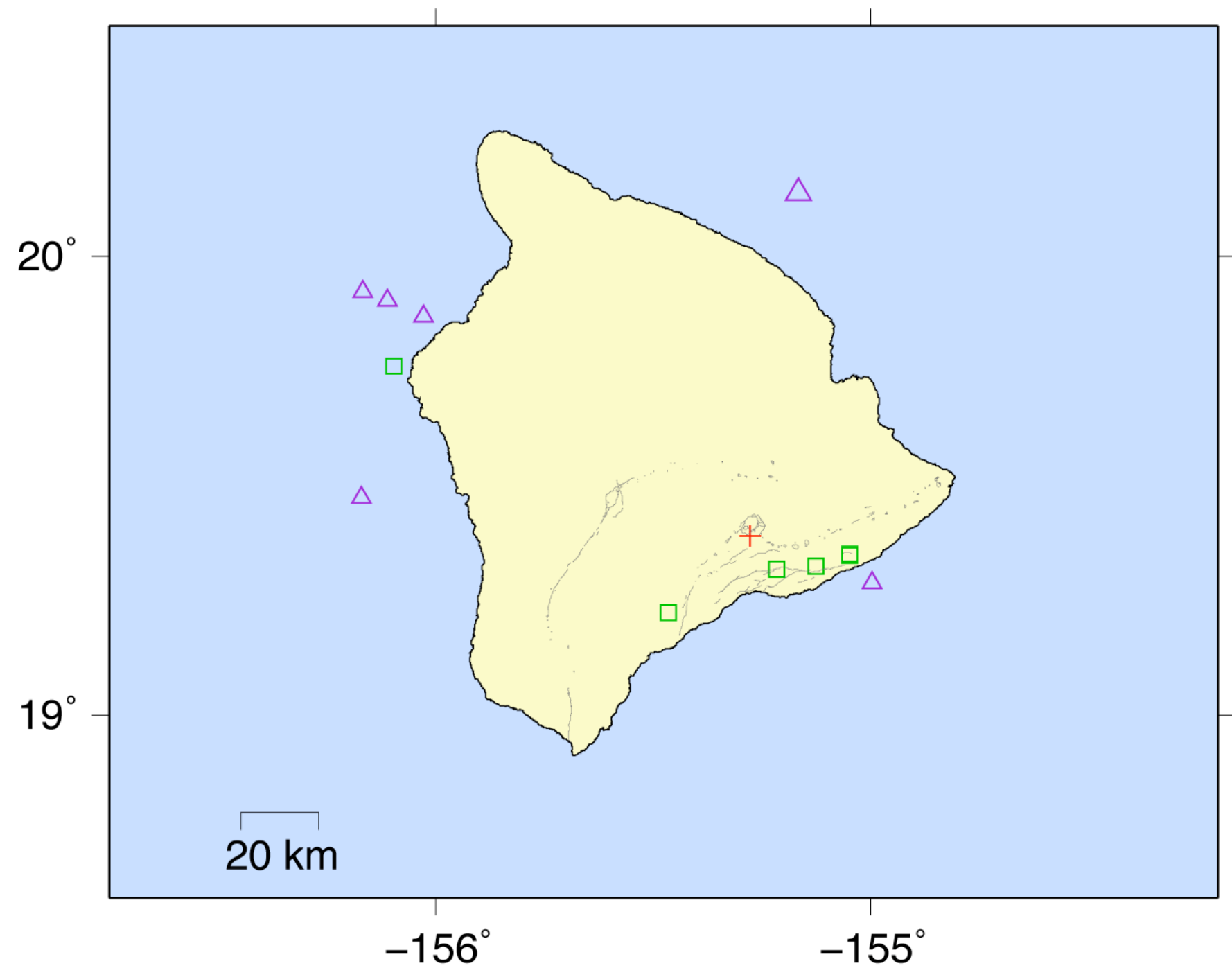

\begin{tabular}{|l|l|l|l|}
\hline$+0.0+\mathrm{km}$ & $\square 5.0+\mathrm{km}$ & $\diamond 13.0+\mathrm{km}$ & $\triangle 20.0+\mathrm{km}$ \\
\hline$\square \mathrm{M} 3.0+$ & $\square \mathrm{M} \mathrm{4.0+}$ & $\square \mathrm{M} 5.0+$ & $\square \mathrm{M} \mathrm{6.0+}$ \\
\hline
\end{tabular}




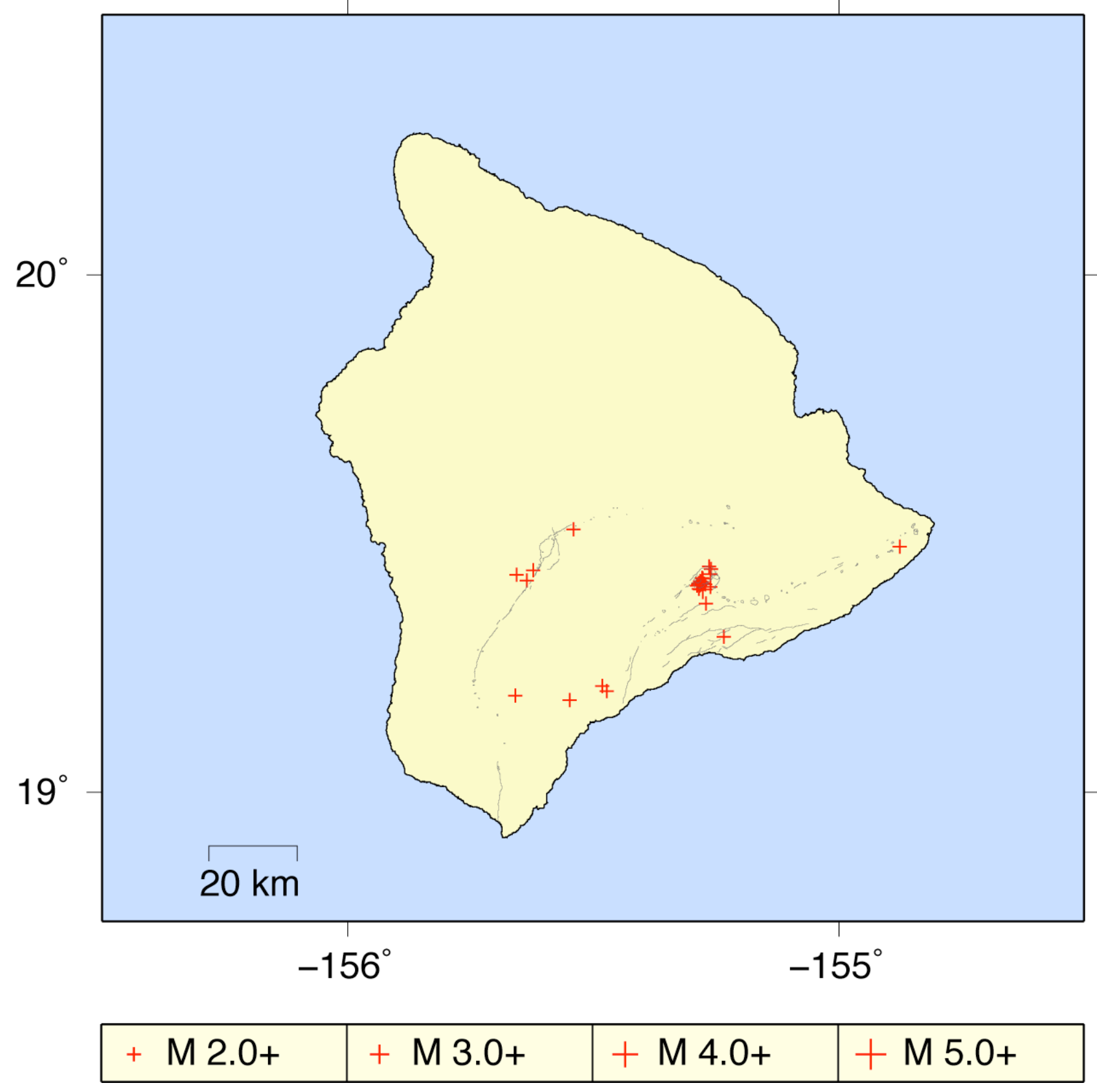




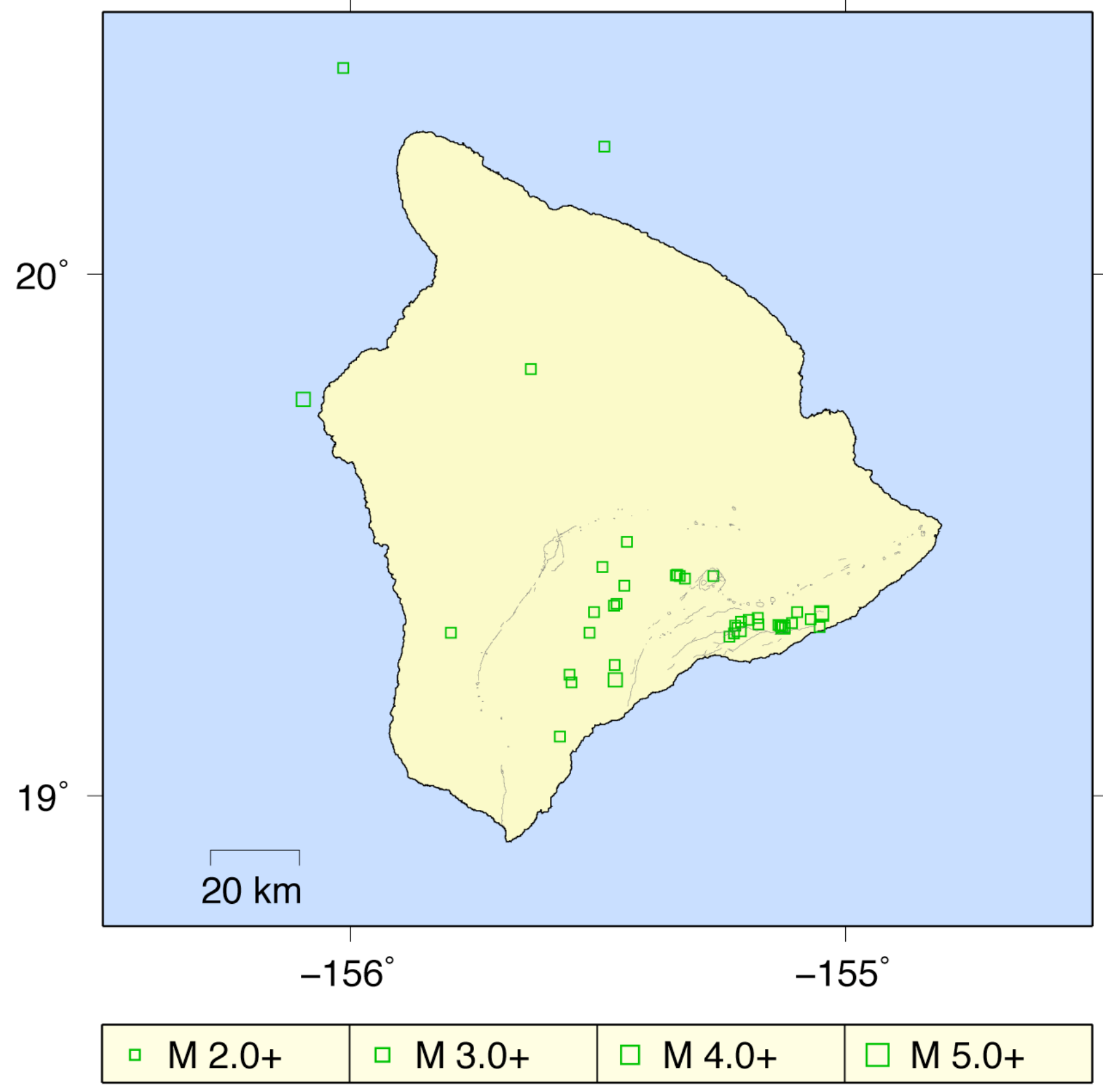




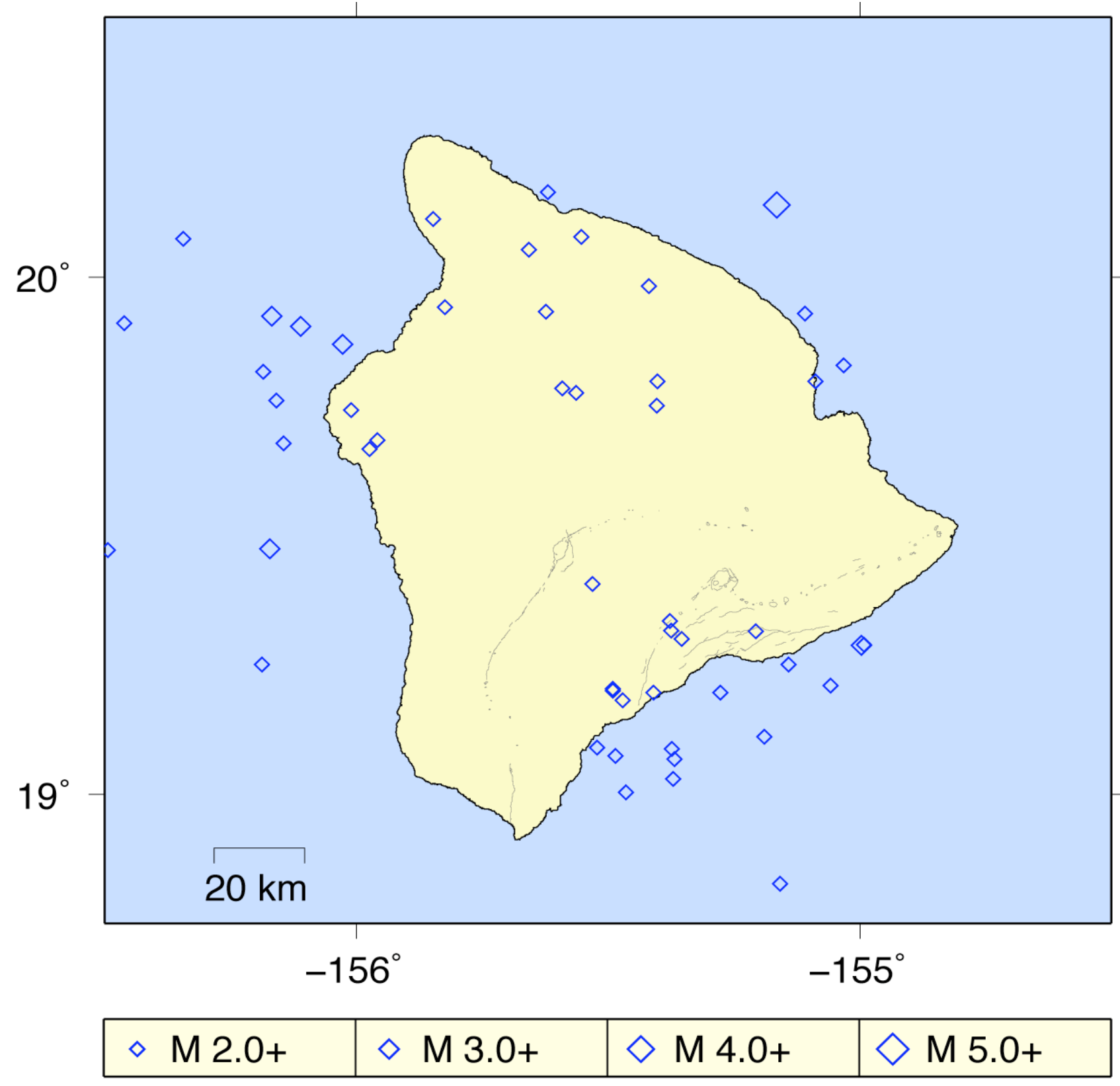

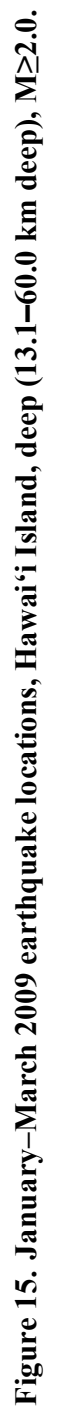




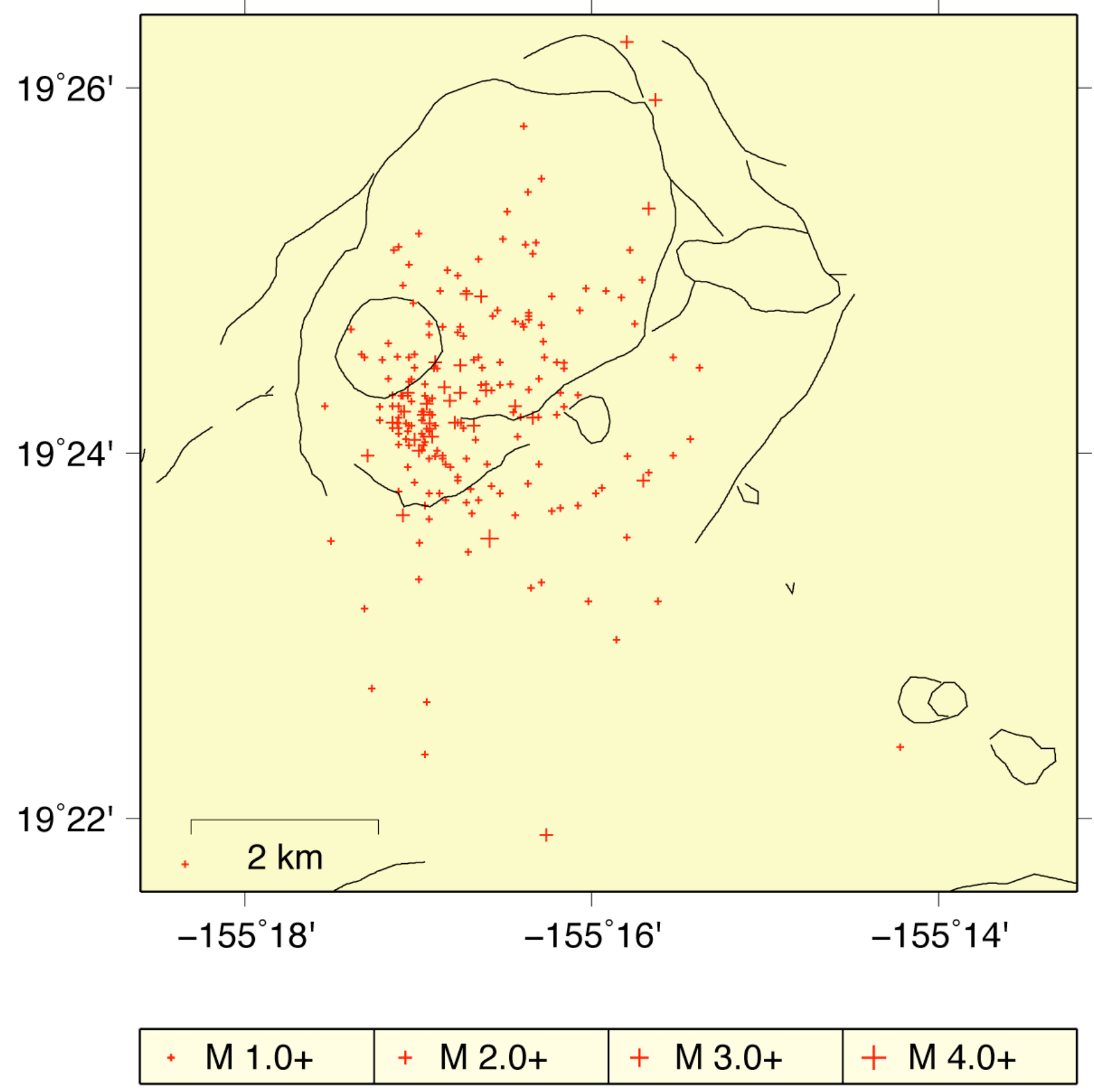

泀 


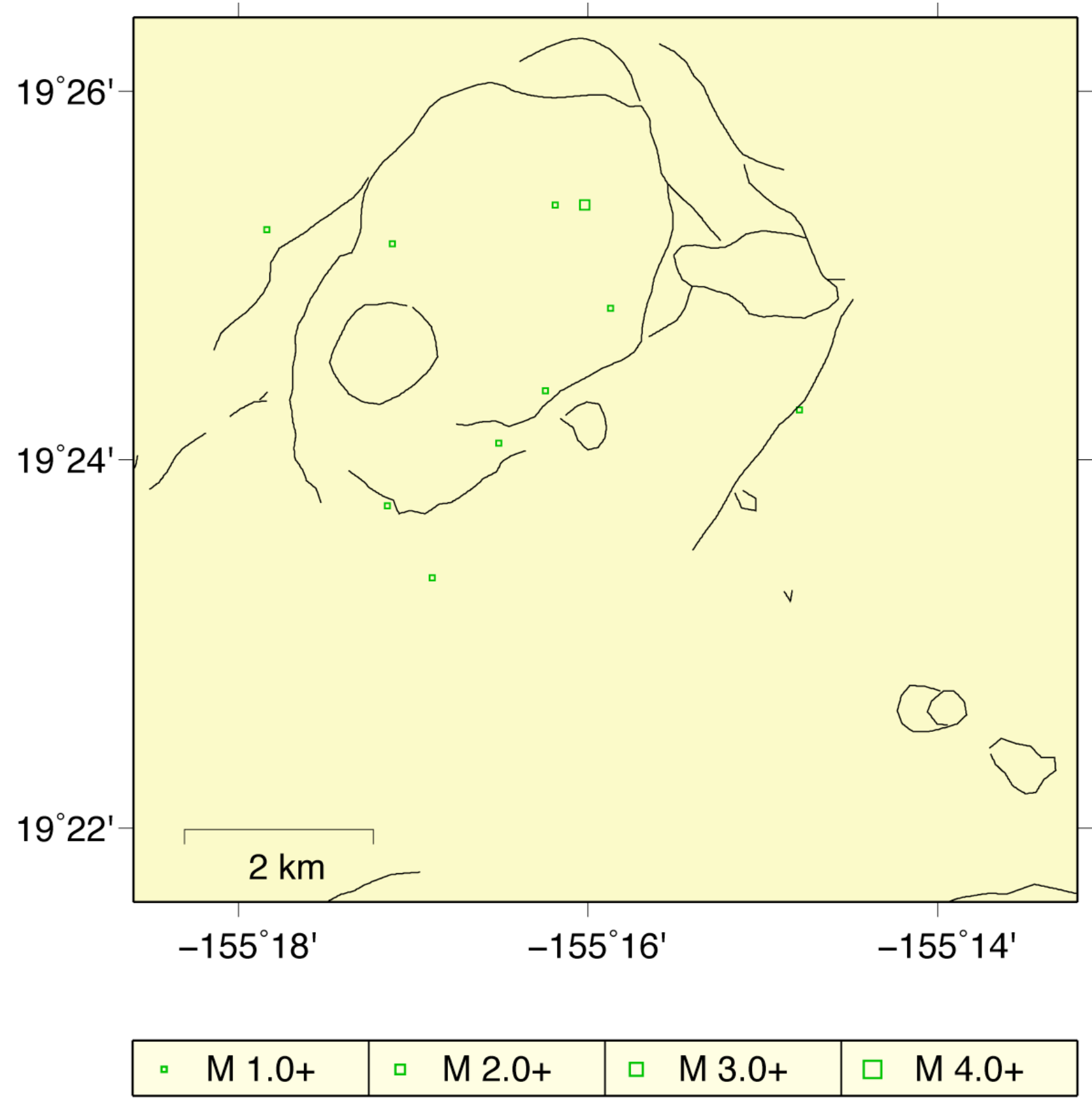




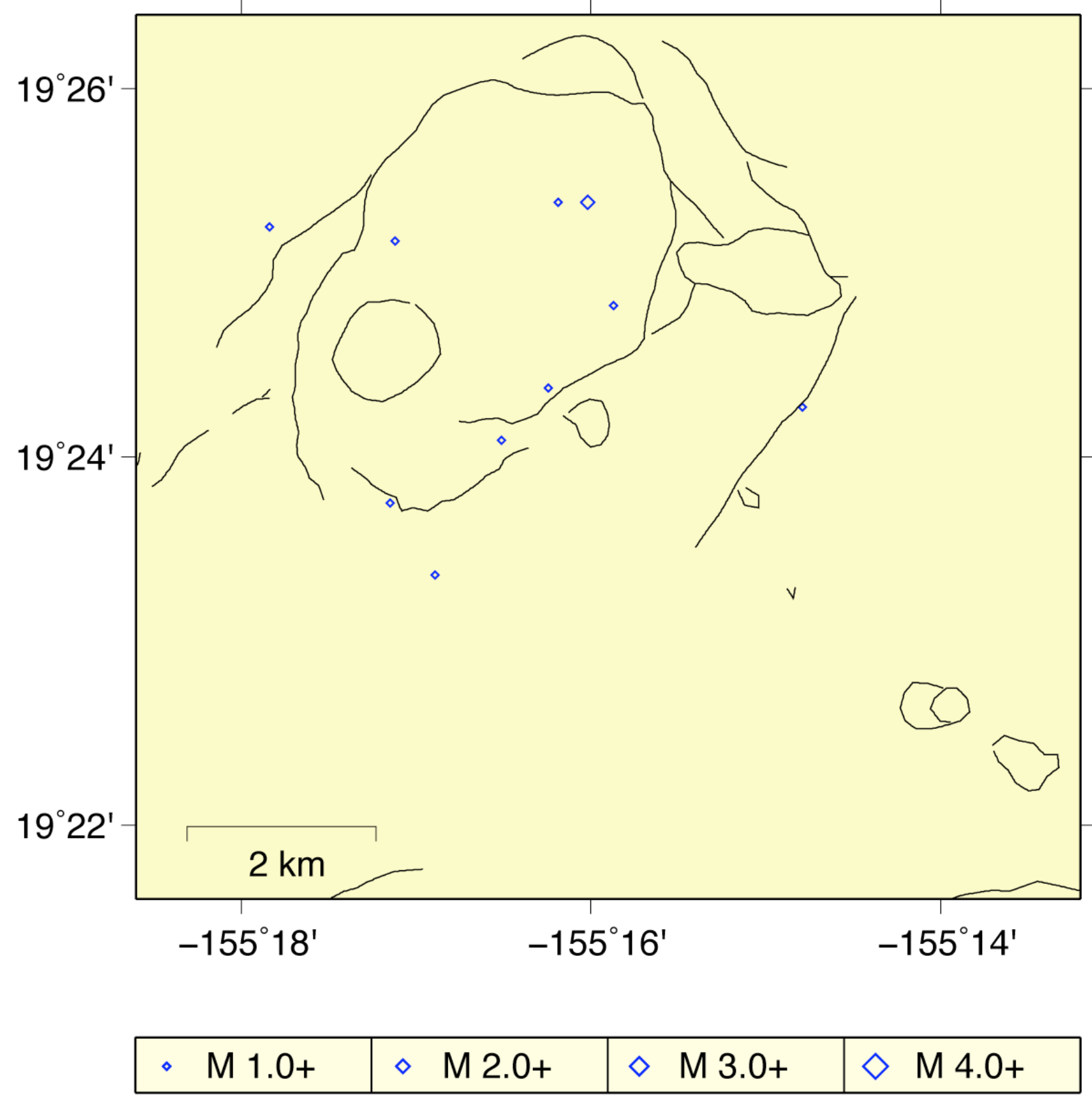




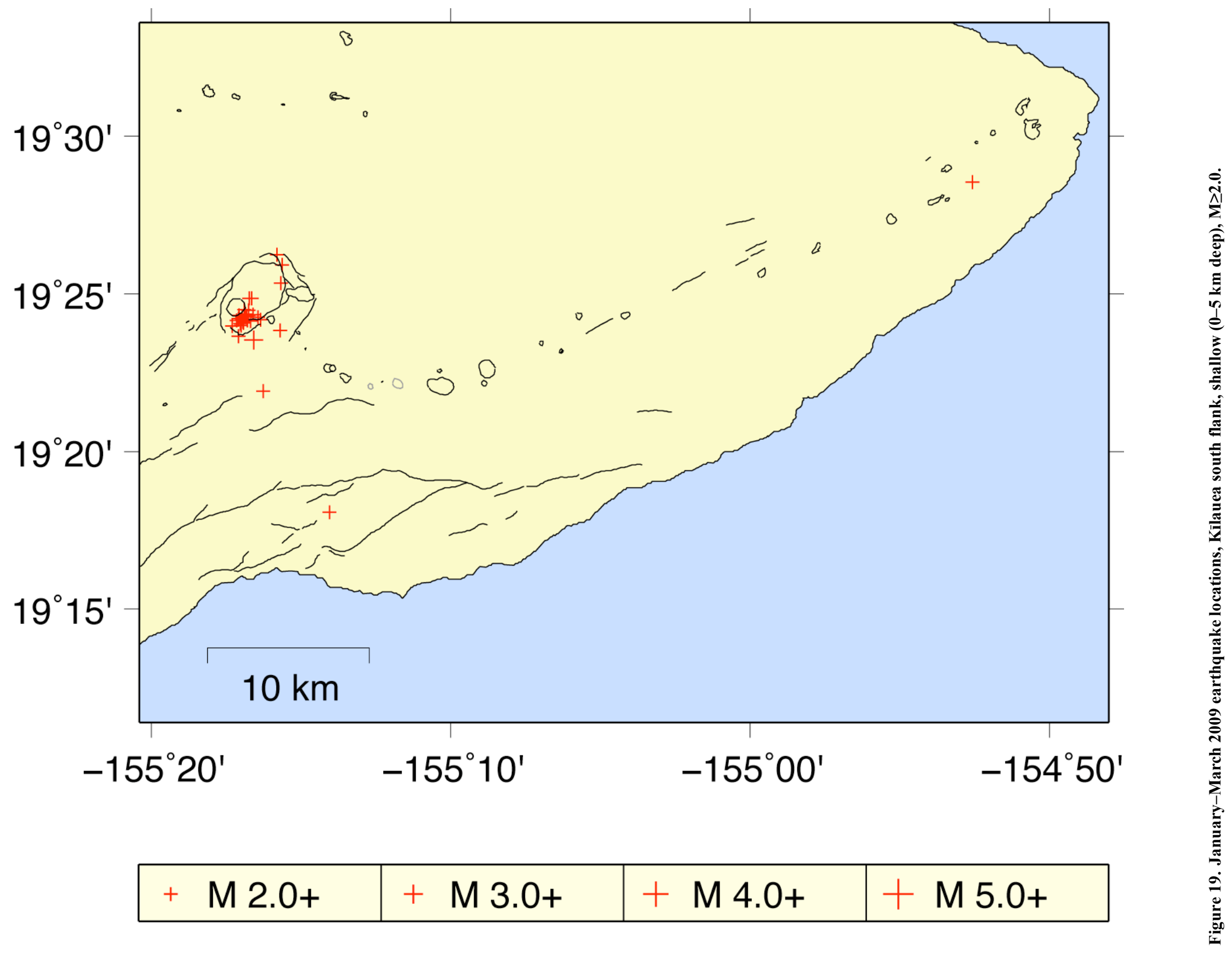




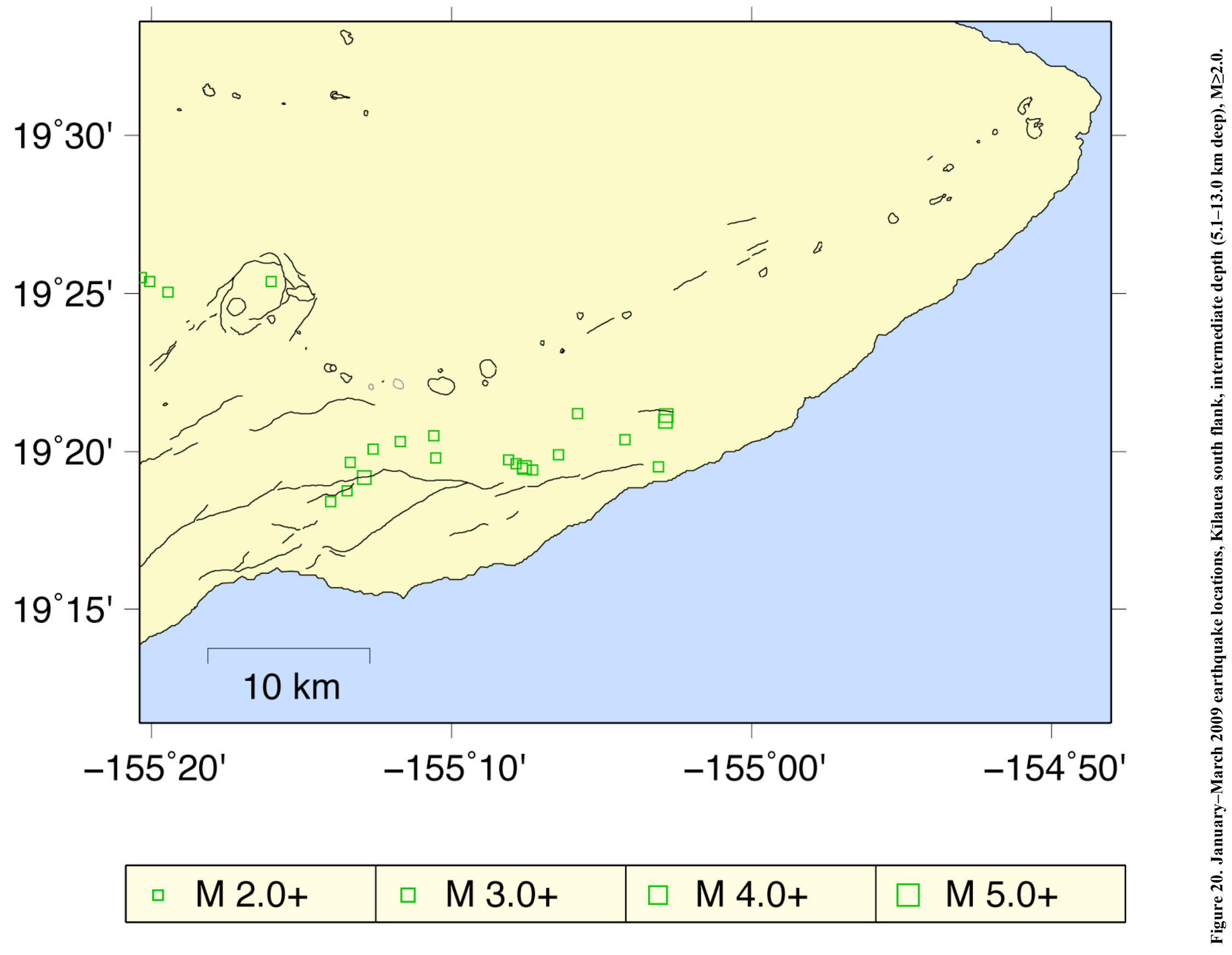




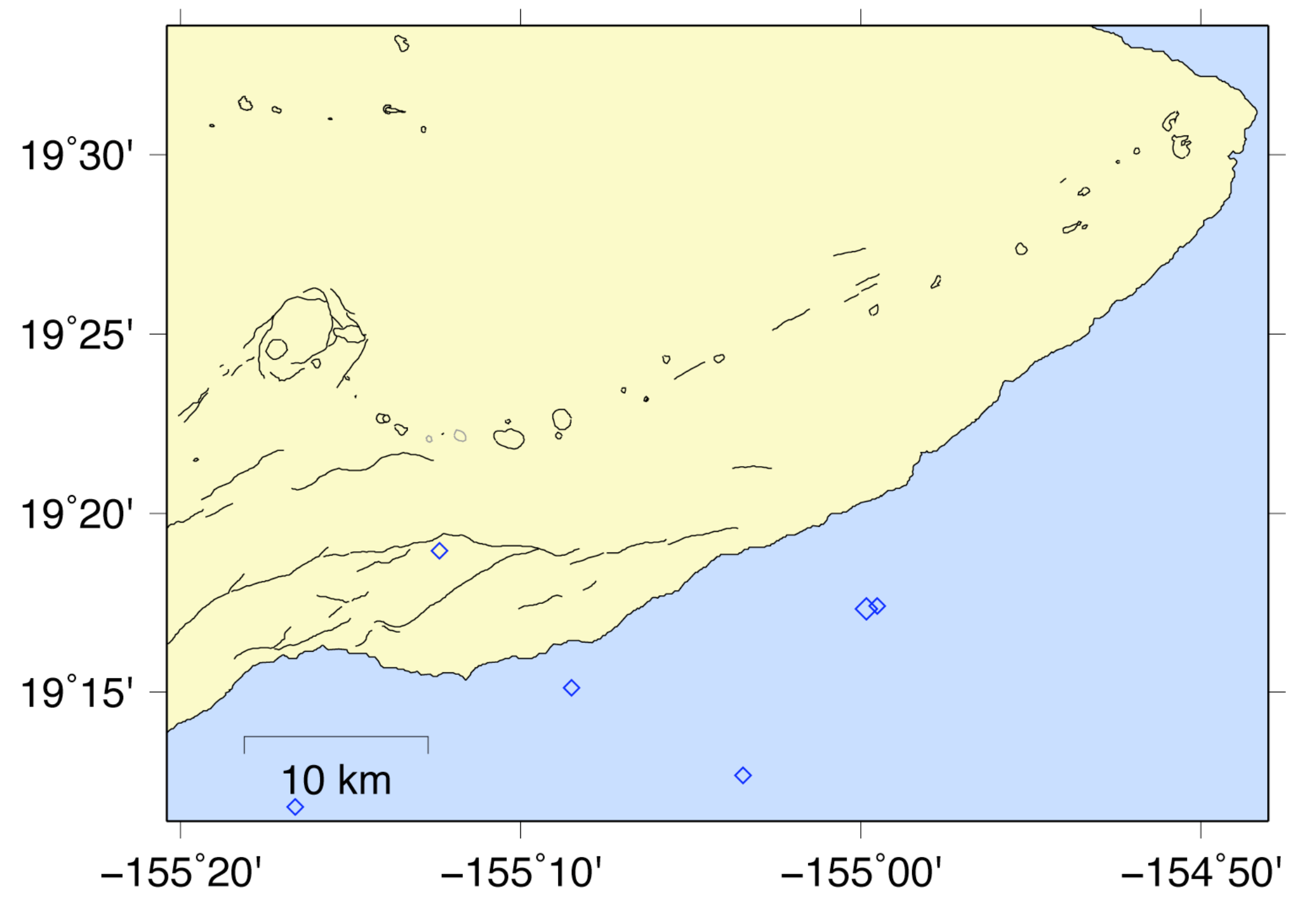

\begin{tabular}{|l|l|l|l|}
\hline$\diamond \mathrm{M} 2.0+$ & $\diamond \mathrm{M} \mathrm{3.0+}$ & $\diamond \mathrm{M} \mathrm{4.0+}$ & $\diamond \mathrm{M} 5.0+$ \\
\hline
\end{tabular}




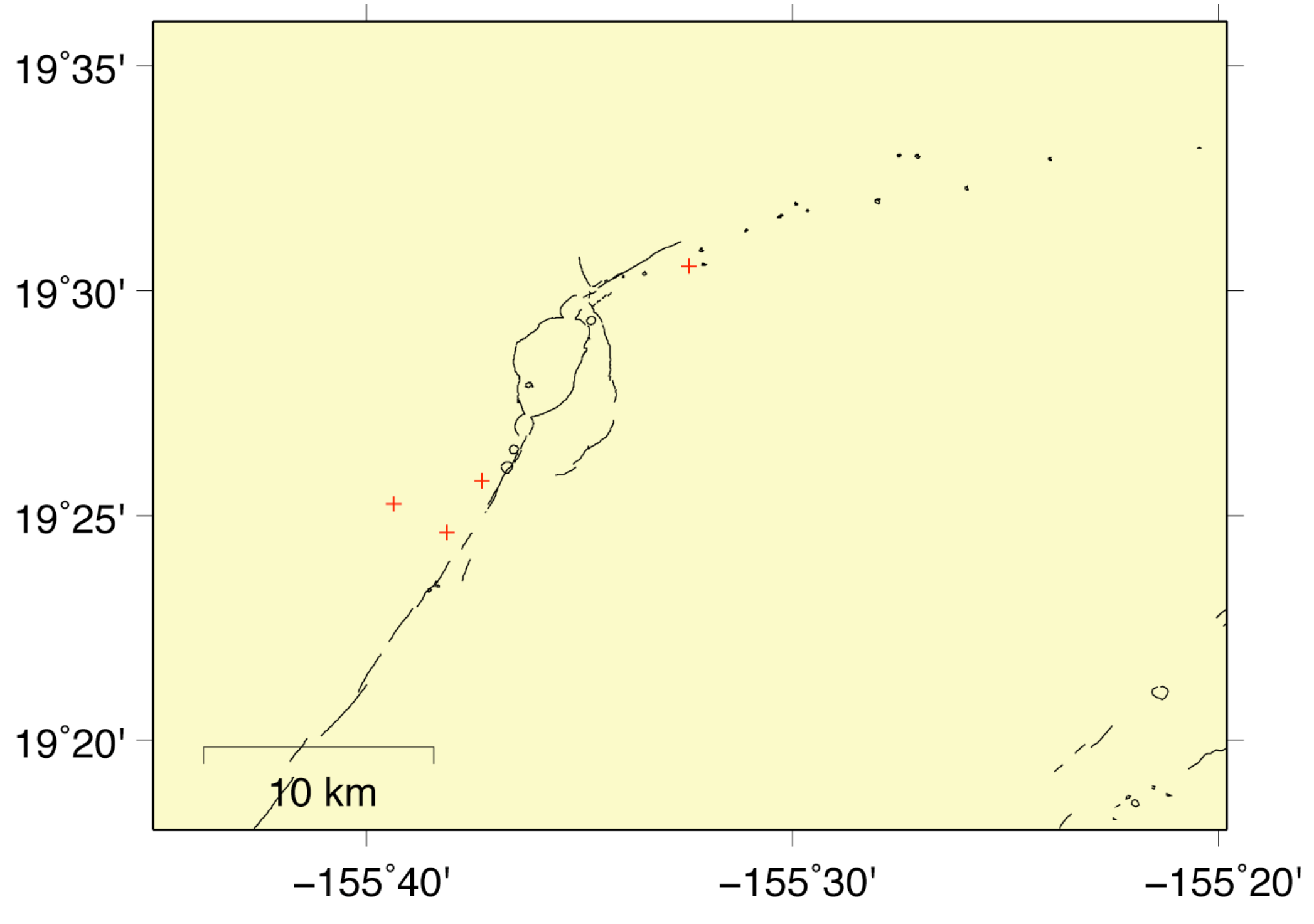

\begin{tabular}{|l|l|l|l|}
\hline$+\mathrm{M} 2.0+$ & $+\mathrm{M} 3.0+$ & $+\mathrm{M} 4.0_{+}$ & $+\mathrm{M} 5.0_{+}$ \\
\hline
\end{tabular}




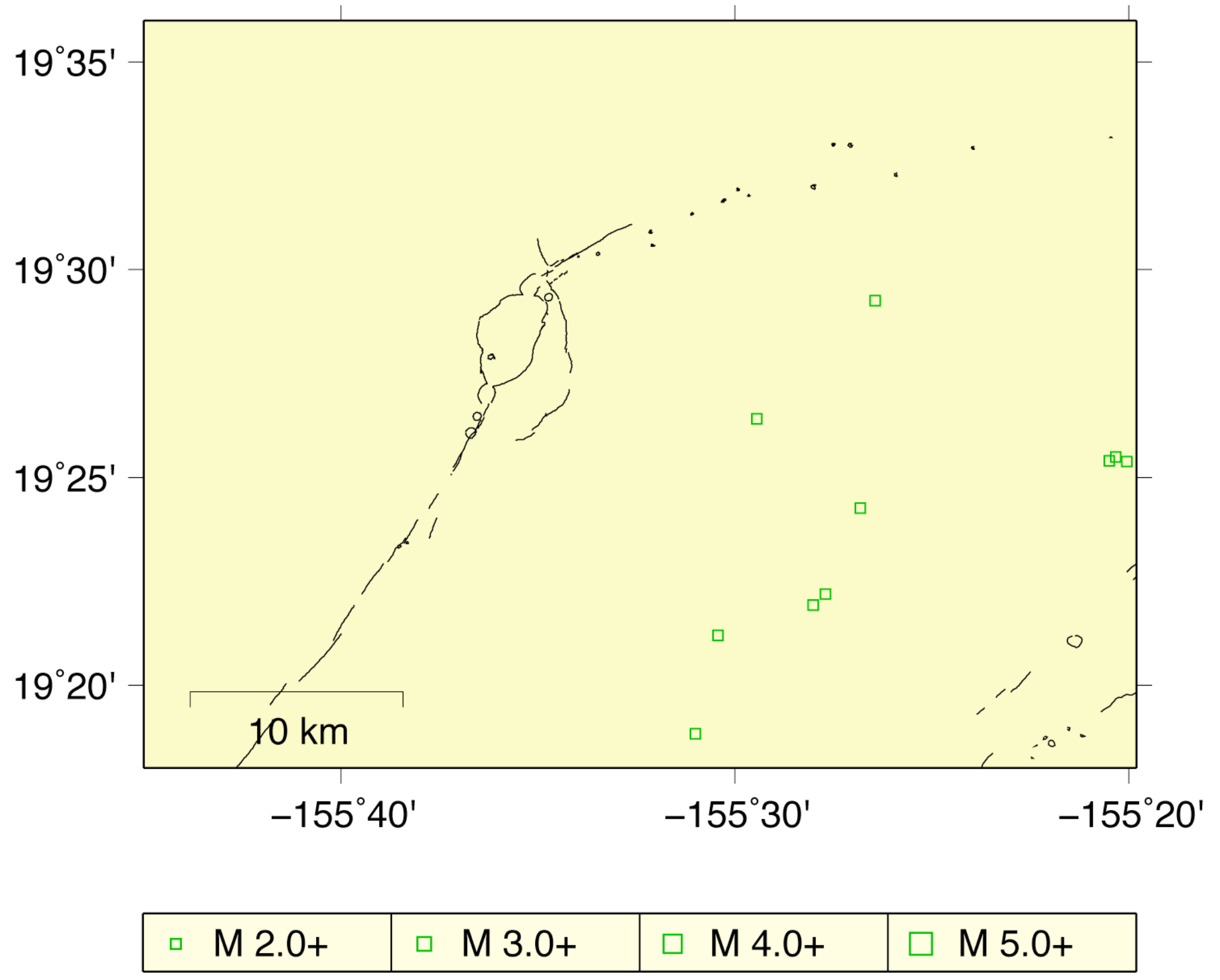




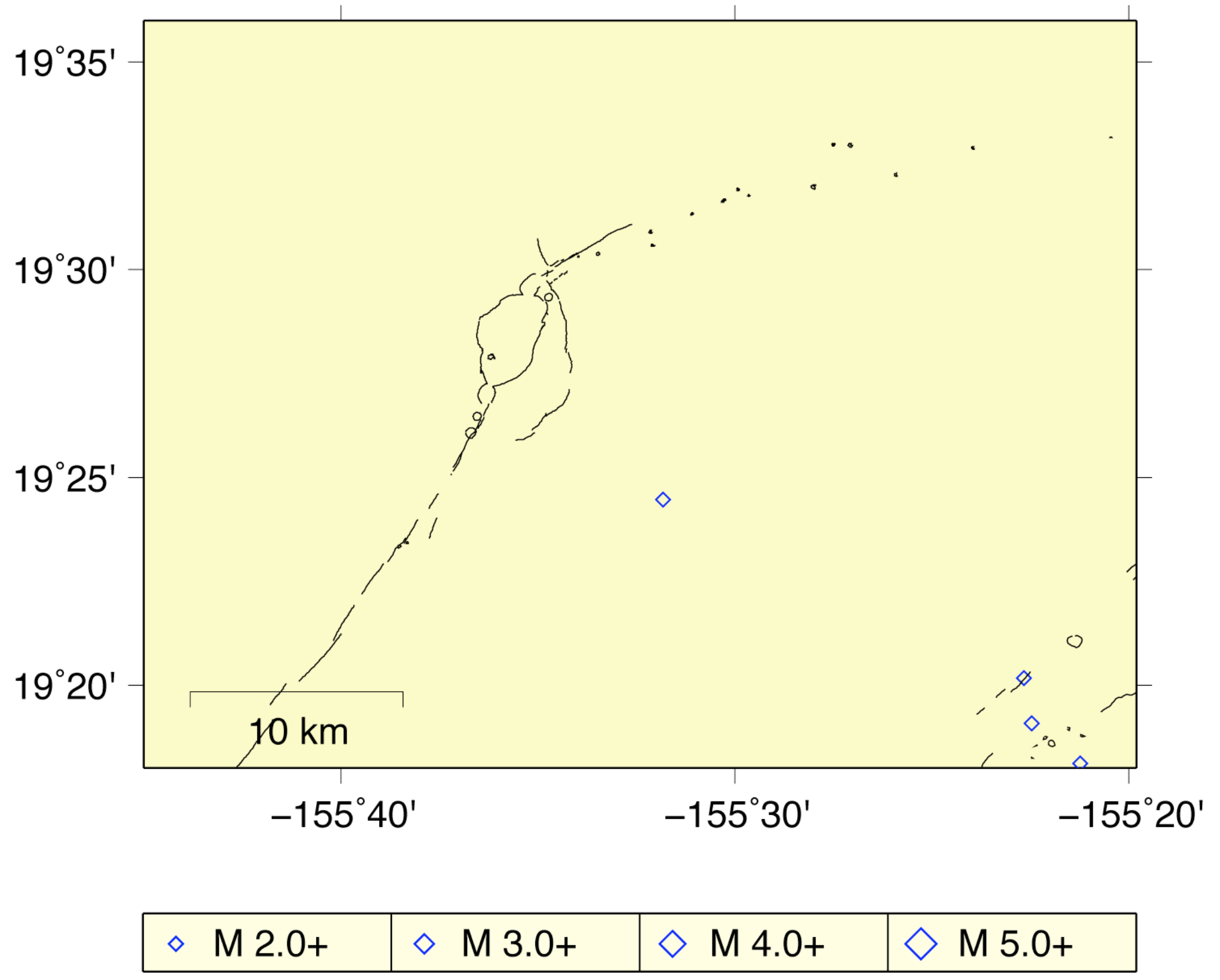


Table 4. Chronological list of the 666 selected events successfully located in CUSP during Januanry-March 2009. Summary data files are available online at http://www.ncedc.org/anss/catalog-search.html (last accessed 02/24/2010). For each event, the following data are presented.

ORIGIN TIME — in Hawaiian Standard Time: date, hour (HR), minute (MN), and second (SEC).

EPICENTER - in degrees and minutes of north latitude (LAT N) and west longitude (LON W) in Old Hawaiian Datum.

DEPTH—depth of focus, in kilometers.

NRD—number of $\mathrm{P} \& \mathrm{~S}$ arrival times with final weights $>0.1$.

NS—number of S arrival times with final weights $>0.1$.

RMS SEC — root mean square travel time residuals, in seconds.

ERH km—standard error of the epicenter, in kilometers.

ERZ $\mathrm{km}$ - standard error of depth of focus, in kilometers.

LOC REMKS - remarks, three-letter code for geographic location of events. See Figures 7-10 for location of mnemonic code. Additional one-letter codes have the following meanings:

F felt,

L long-period character,

$\mathrm{T}$ associated with harmonic tremor,

B quarry or other blast,

\# location program had a convergence problem, which usually means that the depth may be unreliable, and

- $\quad$ depth was held fixed.

PREF MAG-The preferred magnitude chosen from the available magnitudes.

Preference is set as:

$\mathrm{X}$ amplitude magnitude, if none,

D duration magnitude Develocorder equivalent, if none,

$\mathrm{U}$ external magnitude, usually calculated from drum records or from an external source.

AZ GAP — Largest azimuthal gap in degrees between azimuthally adjacent stations.

MIN DS—Distance to the nearest station, in kilometers.

Table 5. List of the 14 events of magnitude 3.0 or greater, selected from table 4.

Figures 25-40. Focal mechanism solutions computed by using the computer program FPFIT (Reasenberg and Oppenheimer, 1985, U.S. Geological Survey Open-File Report 85-739) for events listed in table 5. If calculated, multiple solutions are offered. The solutions are presented without interpretation. The program does not compute mechanism solution for events with additional one-letter codes of $\mathrm{T}$ (tremor) or $\mathrm{B}$ (blast). 


\section{Table 4.}

--ORIGIN TIME (HST)-- -LAT N-- --LON W-- DEPTH N N RMS ERH ERZ LOC PREF N AZ MIN YEAR MON DA HRMN SEC DEG MIN DEG MIN KM RD S SEC KM KM REMKS MAG RD GAP DS

$\begin{array}{lllllllllllllllll}2009 & \text { JAN } & 1 & 0024 & 21.22 & 19 & 24.86 & 155 & 16.23 & 0.48 & 8 & 1.05 & .3 & .7 & \text { SNCL } & 1.1 \mathrm{X}\end{array}$

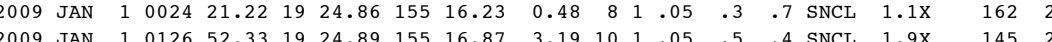

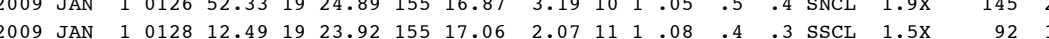

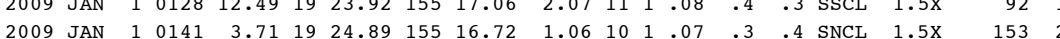

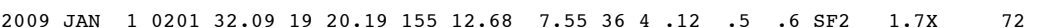

$\begin{array}{llllllllllllllllll}2009 & \text { JAN } & 1 & 0442 & 22.53 & 19 & 24.17 & 155 & 16.77 & 3.36 & 10 & 1 & .06 & .5 & .4 & \text { SSCL } & 1.8 \mathrm{X} & 109\end{array}$

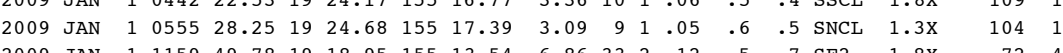
$\begin{array}{lllllllllllllllllll}2009 & \text { JAN } & 1 & 1159 & 49.78 & 19 & 18.95 & 155 & 13.54 & 6.86 & 33 & 2 & .12 & .5 & .7 & \text { SF2 } & 1.8 \mathrm{X} & 72 & 4\end{array}$

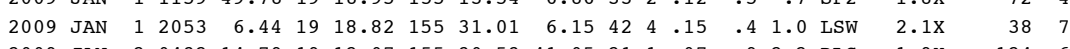

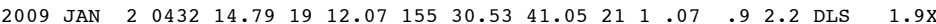

$\begin{array}{lllllllllllllllll}2009 & \text { JAN } & 2 & 0547 & 59.38 & 19 & 23.99 & 155 & 17.29 & 0.80 & 20 & 4 & .12 & .2 & .2 & \text { SSCL } & 2.1 \mathrm{x}\end{array}$ $\begin{array}{lllllllllllllllll}2009 & \text { JAN } & 2 & 0551 & 48.95 & 19 & 24.17 & 155 & 16.79 & 1.35 & 26 & 6 & .10 & .2 & .2 & \text { SSCL } & 2.1 \mathrm{X}\end{array}$ $\begin{array}{llllllllllllllllll}2009 & \text { JAN } & 2 & 0553 & 7.65 & 19 & 24.26 & 155 & 16.44 & 2.13 & 14 & 3 & .08 & .3 & .3 & \text { SECL } & 2.2 \mathrm{U}\end{array}$

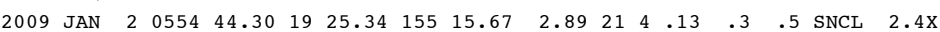

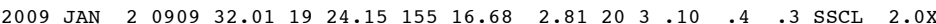

$\begin{array}{lllllllllllllllll}2009 & \text { JAN } & 2 & 1219 & 42.16 & 19 & 14.36 & 155 & 26.68 & 6.22 & 22 & 1 & .14 & .5 & 1.3 & \text { LSW } & 1.5 \mathrm{x}\end{array}$

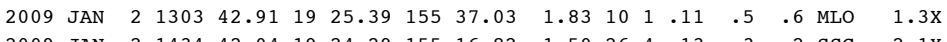

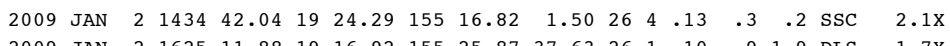

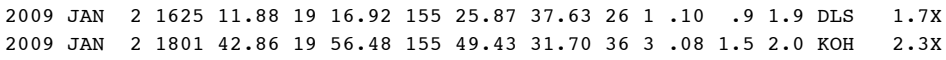

$\begin{array}{lllllllllllllllll}2009 & \text { JAN } & 2 & 2128 & 53.99 & 19 & 20.33 & 155 & 12.81 & 7.44 & 38 & 4 & .13 & .5 & .6 & \mathrm{SF} 2 & 1.8 \mathrm{X}\end{array}$

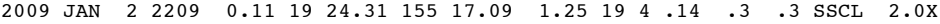

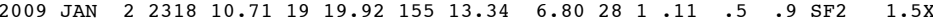

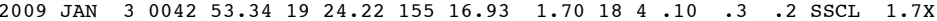

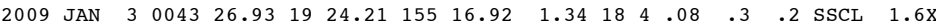

$\begin{array}{lllllllllllllllll}2009 & \text { JAN } & 3 & 0654 & 27.43 & 19 & 17.13 & 155 & 26.03 & 38.52 & 28 & 1 & .11 & .7 & 1.7 & \text { DLS } & 1.7 \mathrm{x}\end{array}$

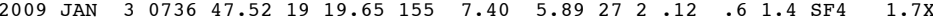
$\begin{array}{lllllllllllllllll}2009 & \text { JAN } & 3 & 1058 & 23.35 & 19 & 13.43 & 155 & 28.26 & 10.39 & 19 & 1 & .12 & .6 & 1.1 & \text { LSW } & 1.7 \mathrm{X}\end{array}$ $\begin{array}{lllllll}26 & 2 & .10 & .6 & 1.0 & \text { SF3 } & 1.6 \mathrm{X}\end{array}$

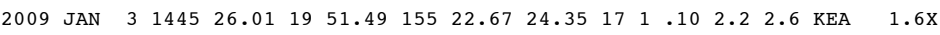

$\begin{array}{lllllllllllllllll}2009 & \text { JAN } & 3 & 1529 & 14.17 & 19 & 22.43 & 155 & 2.61 & 7.46 & 21 & 2 & .13 & .8 & .7 & \text { SF5 } & 1.5 \mathrm{X}\end{array}$

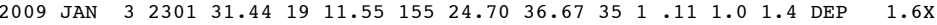

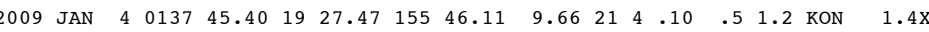

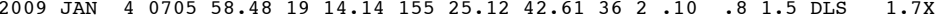
2009 JAN $4 \begin{array}{llllllllllllllll}074.36 & 19 & 20.83 & 155 & 23.97 & 9.63 & 29 & 2 & .11 & .5 & .6 & \text { SWR } & 1.7 \mathrm{X}\end{array}$

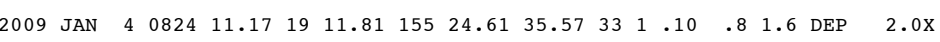
2009 JAN $4 \begin{array}{lllllllllllllll}1945 & 28.56 & 19 & 23.75 & 155 & 17.15 & 5.42 & 14 & 3 & .09 & .7 & .7 & \text { INTL } & 1.9 \mathrm{X}\end{array}$

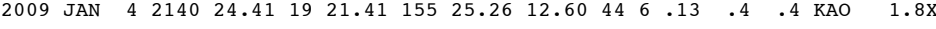

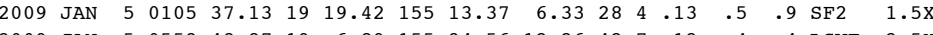
2009 JAN $5 \begin{array}{llllllllllllll}0553 & 48.87 & 19 & 6.89 & 155 & 34.56 & 12.26 & 42 & 7 & .12 & .4 & .4 & \text { LSWF } & 2.5 \mathrm{x}\end{array}$

$\begin{array}{llllllllllllllll}2009 & \text { JAN } & 5 & 0656 & 41.79 & 19 & 6.78 & 155 & 34.29 & 11.61 & 14 & .15 & 1.1 & .9 & \text { LSW } & 1.4 \mathrm{X}\end{array}$

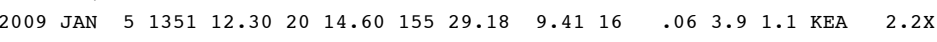
$\begin{array}{llllllllllllllllll}2009 & \text { JAN } & 5 & 1716 & 35.65 & 19 & 30.56 & 155 & 32.43 & 4.56 & 43 & 9 & .14 & .4 & 2.9 & \text { MLO } & 2.3 \mathrm{X} \\ 2009 & \text { JAN } & 5 & 2046 & 15.55 & 19 & 25.88 & 15 & 37.20 & 2.59 & 16 & 2 & .11 & .4 & .5 & \text { MLO } & 1.3 \mathrm{X}\end{array}$ $\begin{array}{lllllllllllllllll}2009 & \text { JAN } & 5 & 2046 & 15.55 & 19 & 25.88 & 155 & 37.20 & 2.59 & 16 & 2 & .11 & .4 & .5 & \text { MLO } & 1.3 \mathrm{X} \\ 2009 & \text { JAN } & 6 & 0023 & 53.85 & 19 & 28.22 & 155 & 13.49 & 36.91 & 4610 & .10 & .6 & .9 & \text { DEP } & 2.0 \mathrm{X}\end{array}$
---ORIGIN TIME (HST)-- -LAT N-- --LON W-- DEPTH N N RMS ERH ERZ LOC PREF N AZ MIN YEAR MON DA HRMN SEC DEG MIN DEG MIN KM RD S SEC KM KM REMKS MAG RD GAP DS

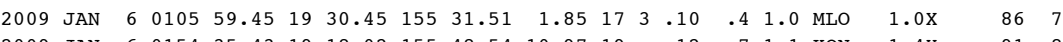

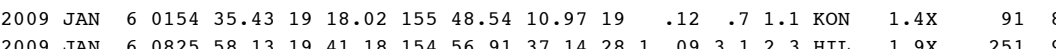

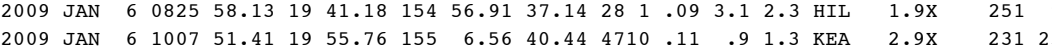

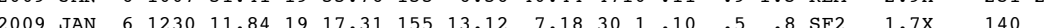

$\begin{array}{lllllllllllllllllll}2009 & \text { JAN } & 6 & 1413 & 0.58 & 19 & 22.61 & 155 & 29.76 & 9.15 & 21 & 1 & .07 & .4 & 1.0 & \text { KAO } & 1.7 \mathrm{X} & 70 & 4\end{array}$

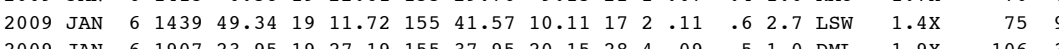
$\begin{array}{lllllllllllllllllll}2009 & \text { JAN } & 6 & 1907 & 23.95 & 19 & 27.19 & 155 & 37.95 & 20.15 & 28 & 4 & .09 & .5 & 1.0 & \text { DML } & 1.9 \mathrm{X} & 106 & 3\end{array}$

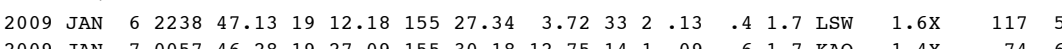
$\begin{array}{lllllllllllllllllll}2009 & \text { JAN } & 7 & 0057 & 46.28 & 19 & 27.09 & 155 & 30.18 & 12.75 & 14 & 1 & .09 & .6 & 1.7 & \text { KAO } & 1.4 \mathrm{X} & 74 & 6\end{array}$

$\begin{array}{llllllllllllllllllll}2009 & \text { JAN } & 7 & 0631 & 53.02 & 19 & 24.86 & 155 & 16.64 & 2.76 & 21 & 4 & .10 & .3 & .2 & \text { SNCL } & 2.2 \mathrm{X} & 143 & 2\end{array}$

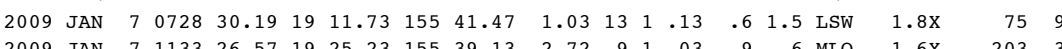

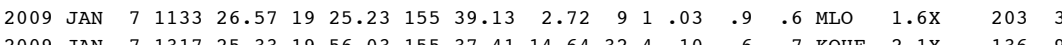
$\begin{array}{lllllllllllllllllll}2009 & \text { JAN } & 7 & 1317 & 25.33 & 19 & 56.03 & 155 & 37.41 & 14.64 & 32 & 4 & .10 & .6 & .7 & \text { KOHF } & 2.1 \mathrm{X} & 136 & 9 \\ 2009 & \text { JAN } & 7 & 1805 & 52.54 & 19 & 19.23 & 155 & 11.25 & 6.35 & 28 & 1 & .11 & .5 & .8 & \text { SF } 3 & 1.6 \mathrm{X} & 103 & 6\end{array}$

$\begin{array}{lllllllllllllllllll}2009 & \text { JAN } & 8 & 0242 & 35.33 & 19 & 23.89 & 155 & 26.95 & 9.54 & 19 & 1 & .12 & .5 & 1.0 & \text { KAO } & 1.4 \mathrm{X} & 61\end{array}$

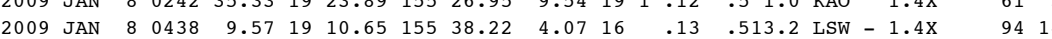
$\begin{array}{llllllllllllllllll}2009 & \text { JAN } & 8 & 0506 & 57.77 & 19 & 16.54 & 155 & 22.84 & 3.17 & 20 & 2 & .13 & .4 & 1.3 & \text { SWR } & 1.4 \mathrm{X} & 121\end{array}$ $\begin{array}{llllllllllllllllll}2009 & \text { JAN } & 8 & 0605 & 0.49 & 19 & 20.17 & 155 & 22.66 & 34.07 & 47 & 8 & .11 & .6 & .9 & \text { DEP } & 2.2 \mathrm{X} & 74\end{array}$

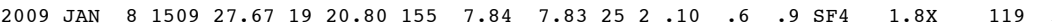

$\begin{array}{lllllllllllllllllll}2009 & \text { JAN } & 8 & 2253 & 5.42 & 19 & 16.53 & 155 & 12.12 & 8.60 & 26 & 3 & .12 & .7 & .6 & \mathrm{SF} 3 & 1.4 \mathrm{X} & 203 & 3\end{array}$

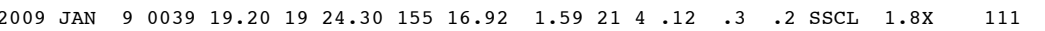

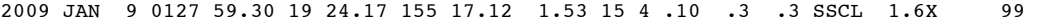

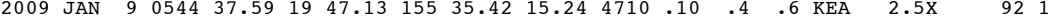

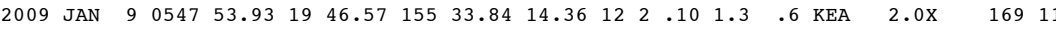

$\begin{array}{llllllllllllllllll}2009 & \text { JAN } & 9 & 0712 & 21.55 & 19 & 21.49 & 155 & 5.57 & 7.22 & 29 & 3 & .13 & .6 & .8 & \mathrm{SF} 4 & 1.7 \mathrm{x} & 149\end{array}$

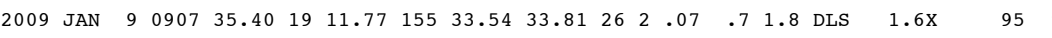

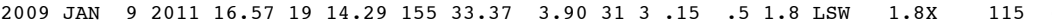

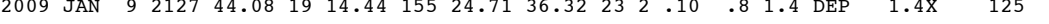

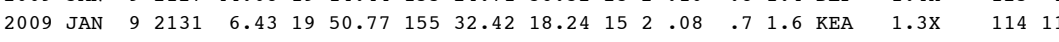

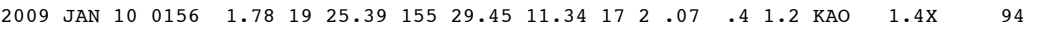

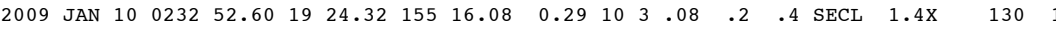

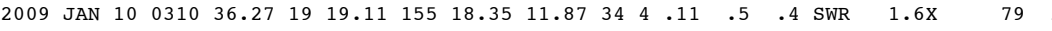

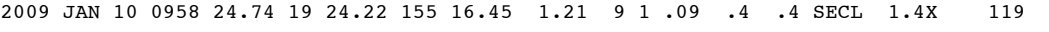

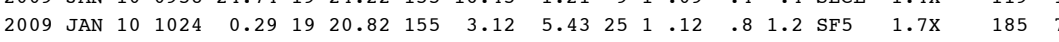

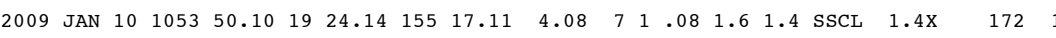

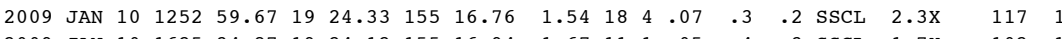

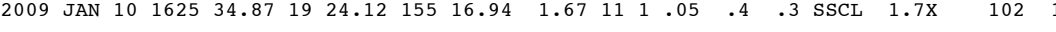

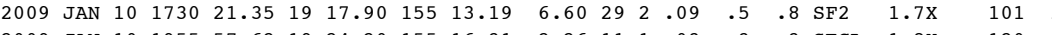

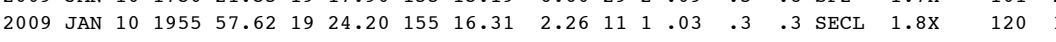

$\begin{array}{llllllllllllllllll}2009 & \text { JAN } 10 & 2053 & 0.30 & 19 & 25.58 & 155 & 24.63 & 9.28 & 39 & 4 & .12 & .4 & .8 & \text { KAO } & 1.9 \mathrm{X} & 47 & 8\end{array}$

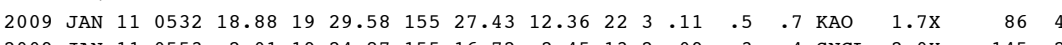
$\begin{array}{lllllllllllllllll}2009 & \text { JAN } 11 & 0553 & 2.01 & 19 & 24.87 & 155 & 16.72 & 2.45 & 13 & 2 & .09 & .3 & .4 & \text { SNCL } & 2.0 \mathrm{X} & 145\end{array}$

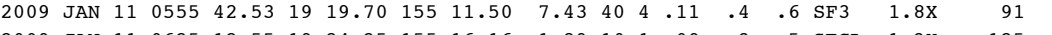


---ORIGIN TIME (HST) -- -LAT N-- --LON W-- DEPTH N N RMS ERH ERZ LOC PREF N AZ MIN YEAR MON DA HRMN SEC DEG MIN DEG MIN KM RD S SEC KM KM REMKS MAG RD GAP DS

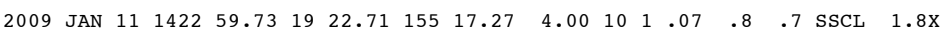
$\begin{array}{llllllllllll}2009 & & \end{array}$

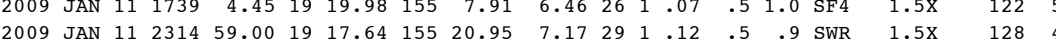

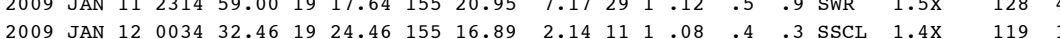

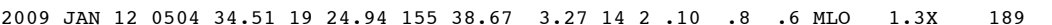

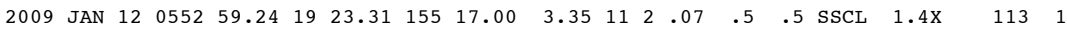

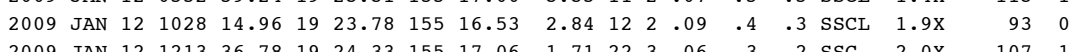

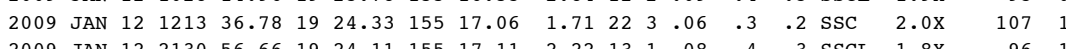

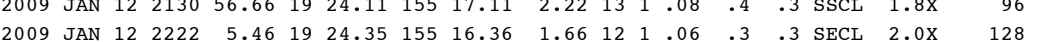

$\begin{array}{llllllllllllllllll}2009 & \text { JAN } 12 & 2327 & 50.56 & 19 & 16.77 & 155 & 34.69 & 4.91 & 31 & 1 & .17 & .5 & 1.9 & \text { LSW } & 1.8 \mathrm{X} & 97 & 9\end{array}$

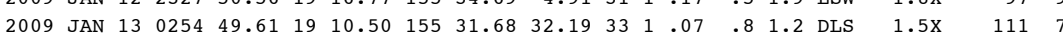

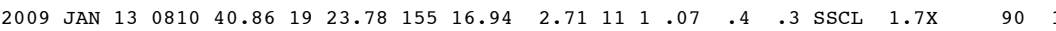

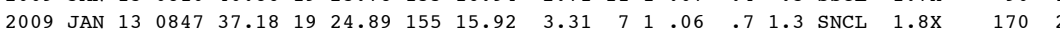

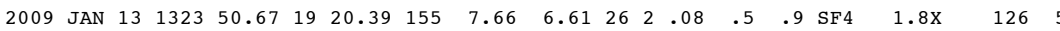

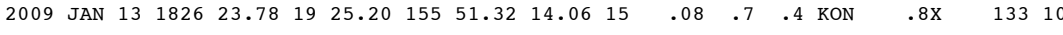

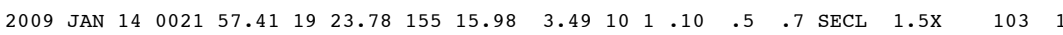

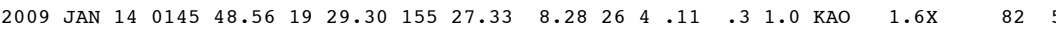

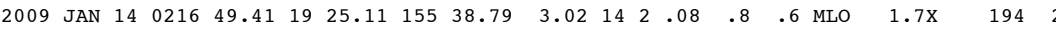

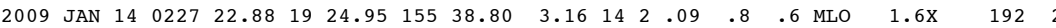

$\begin{array}{lllllllllllllllll}2009 & \text { JAN } 14 & 0234 & 0.36 & 19 & 25.38 & 155 & 20.06 & 9.05 & 45 & 7 & .10 & .3 & .6 & \text { KAO } & 2.6 \mathrm{X} & 47\end{array}$

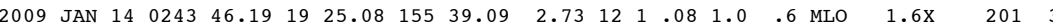

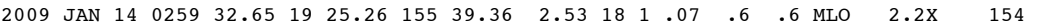

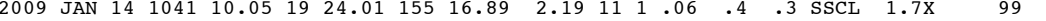

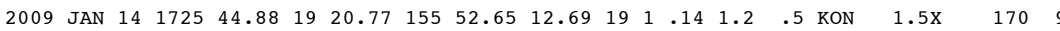

$\begin{array}{llllllllllllllllll}2009 & \text { JAN } 14 & 2117 & 31.66 & 19 & 24.41 & 155 & 16.30 & 2.21 & 10 & 1 & .05 & .3 & .4 & \text { SECL } & 1.8 \mathrm{X} & 131 & 1\end{array}$

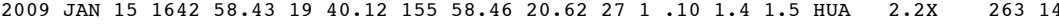

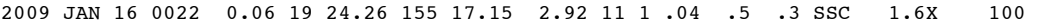

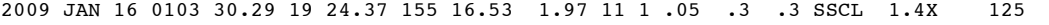

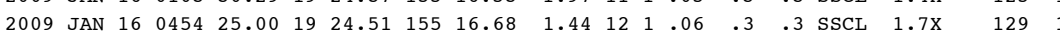

$\begin{array}{llllllllllllllllll}2009 & \text { JAN } 16 & 0510 & 17.05 & 19 & 24.37 & 155 & 16.64 & 2.95 & 10 & 1 & .06 & .4 & .5 & \text { SSCL } & 1.5 \mathrm{X} & 122 & 1\end{array}$

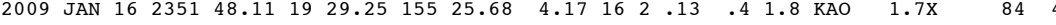

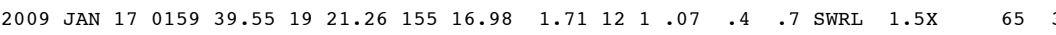

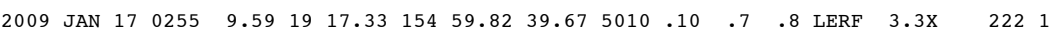

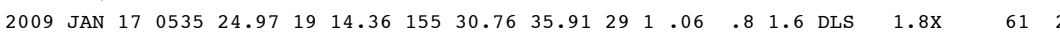

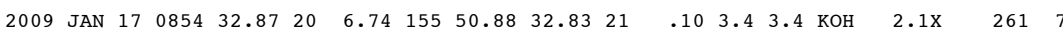
$\begin{array}{llllllllllllllllll}2009 & \text { JAN } 17 & 1209 & 0.72 & 19 & 24.20 & 155 & 16.41 & 1.40 & 10 & 1 & .04 & .3 & .3 & \text { SECL } & 1.6 \mathrm{X} & 119 & 1\end{array}$

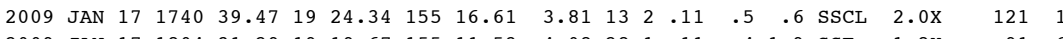

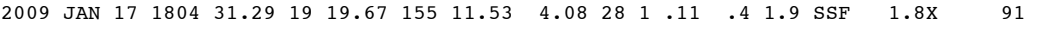

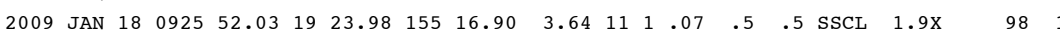

$\begin{array}{llllllllllllllllll}2009 & \text { JAN } 18 & 0950 & 51.89 & 19 & 23.64 & 155 & 16.94 & 3.62 & 10 & 1 & .08 & .6 & .6 & \text { SSCL } & 1.5 \mathrm{x} & 69 & 0\end{array}$ $\begin{array}{llllllllllllllllll}2009 & \text { JAN } 18 & 1336 & 25.04 & 19 & 18.81 & 155 & 47.77 & 10.47 & 38 & 5 & .14 & .4 & .6 & \text { KON } & 2.4 \mathrm{X} & 90 & 9\end{array}$ $\begin{array}{llllllllllllllllll}2009 & \text { JAN } 18 & 1427 & 41.15 & 19 & 24.27 & 155 & 16.95 & 2.25 & 18 & 3 & .07 & .3 & .3 & \text { SSCL } & 2.2 \mathrm{X} & 108 & 1\end{array}$

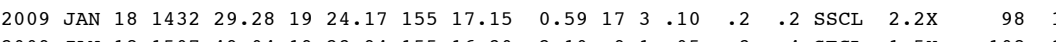

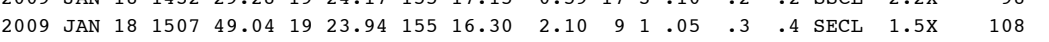

---ORIGIN TIME (HST)-- -LAT N-- --LON W-- DEPTH N N RMS ERH ERZ LOC PREF N AZ MIN YEAR MON DA HRMN SEC DEG MIN DEG MIN KM RD S SEC KM KM REMKS MAG RD GAP DS

$\begin{array}{llllllllllllllllll}2009 & \text { JAN } 18 & 1525 & 52.28 & 19 & 24.31 & 155 & 17.10 & 1.44 & 21 & 3 & .06 & .2 & .2 & \text { SSCL } & 2.0 \mathrm{X} & 105 & 1\end{array}$

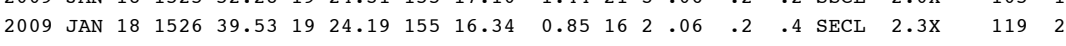

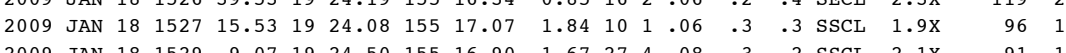

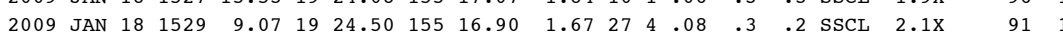

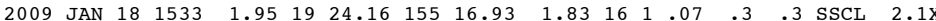

$\begin{array}{llllllllllllllll}2009 & \text { JAN } 18 & 1535 & 3.85 & 19 & 24.17 & 155 & 16.75 & 2.47 & 15 & 1 & .09 & .4 & .3 & \text { SSCL } & 1.8 \mathrm{X}\end{array}$

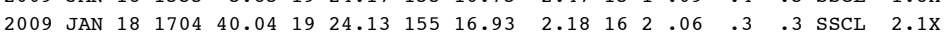

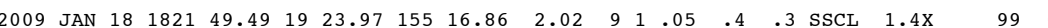

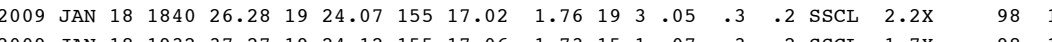
2009 JAN $18 \begin{array}{llllllllllllllll}1932 & 37.27 & 19 & 24.12 & 155 & 17.06 & 1.73 & 15 & 1 & .07 & .3 & .2 & \text { SSCL } & 1.7 \mathrm{X}\end{array}$

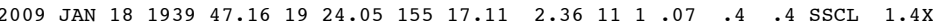

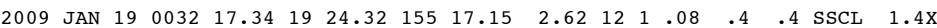

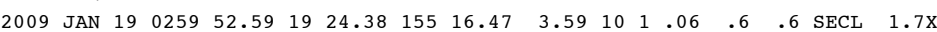

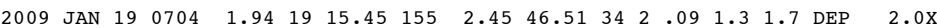

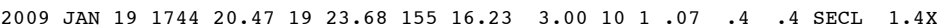

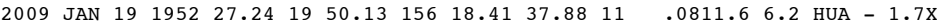

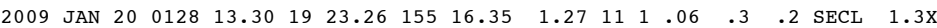

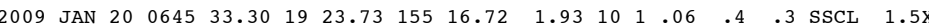

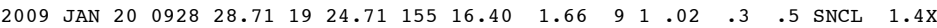

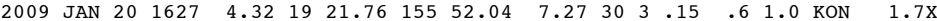

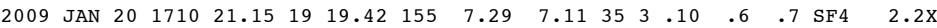
$\begin{array}{llllllllllllllll}2009 & \text { JAN } 20 & 2203 & 39.48 & 19 & 19.07 & 155 & 22.47 & 35.72 & 41 & 5 & .10 & .7 & 1.1 & \text { DEP } & 2.2 \mathrm{X}\end{array}$ $\begin{array}{llllllllllllllll}2009 & \text { JAN } 20 & 2256 & 45.35 & 19 & 18.11 & 155 & 21.24 & 33.03 & 34 & 3 & .10 & .7 & 1.2 & \text { DEP } & 2.3 \mathrm{X}\end{array}$

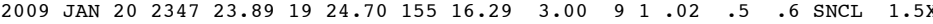
$\begin{array}{lllllllllllllll}2009 & \text { JAN } 21 & 0324 & 10.77 & 19 & 20.60 & 155 & 8.23 & 8.40 & 23 & 1.05 & .5 & .8 & \text { SF } 4 & 1.4 \mathrm{X}\end{array}$

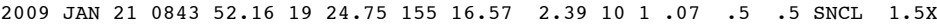

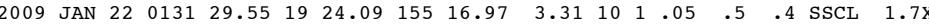

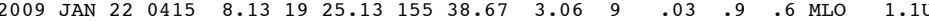

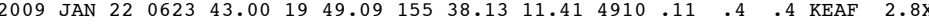
$\begin{array}{llllllllllllll}2009 & \text { JAN } 22 & 1009 & 31.77 & 19 & 21.93 & 155 & 28.02 & 9.91 & 17 & 4 & .10 & .4 & .6 \\ \text { KAO } & 2.3 \mathrm{X}\end{array}$

$\begin{array}{llllllllllllllll}2009 & \text { JAN } 22 & 1350 & 24.59 & 19 & 34.25 & 155 & 41.66 & 7.34 & 28 & 4 & .13 & .5 & 1.7 & \text { MLO } & 1.9 \mathrm{X}\end{array}$

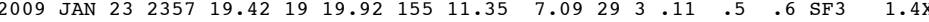

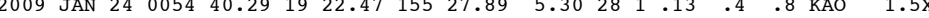
2009 JAN $24 \quad 0448$

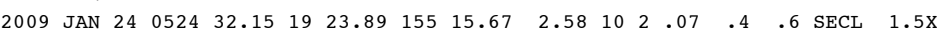

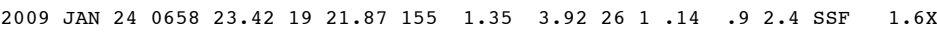

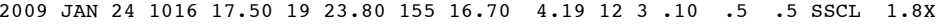

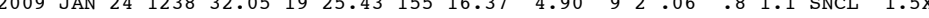

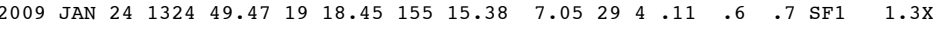

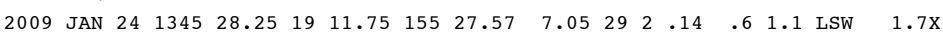

$\begin{array}{llllllllllllllll}2009 & \text { JAN } 25 & 1032 & 46.29 & 19 & 25.11 & 155 & 39.05 & 3.12 & 13 & 2 & .07 & .8 & .7 & \text { MLO } & 1.6 \mathrm{X}\end{array}$

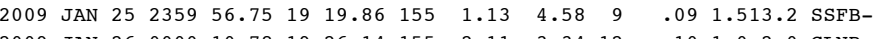

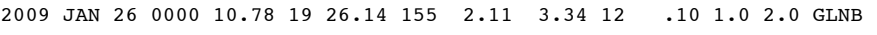

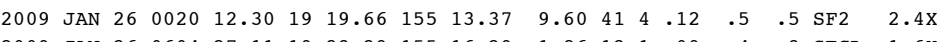

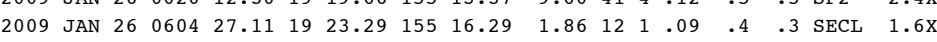


---ORIGIN TIME (HST)-- -LAT N-- --LON W-- DEPTH N N RMS ERH ERZ LOC PREF N AZ MIN YEAR MON DA HRMN SEC DEG MIN DEG MIN KM RD S SEC KM KM REMKS MAG RD GAP DS

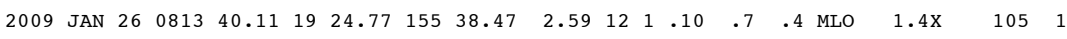

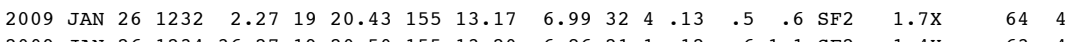

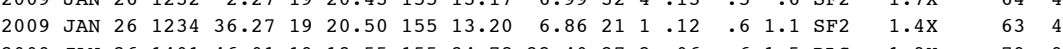

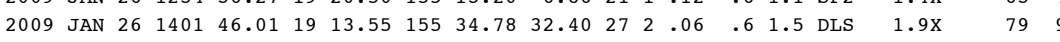

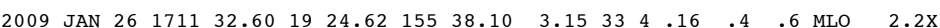

$\begin{array}{llllllllllllllll}2009 & \text { JAN } 26 & 2012 & 11.09 & 19 & 20.80 & 155 & 5.91 & 7.41 & 25 & 2 & .09 & .6 & .8 & \text { SF } 4 & 1.8 \mathrm{X}\end{array}$

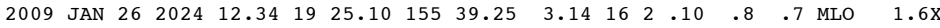

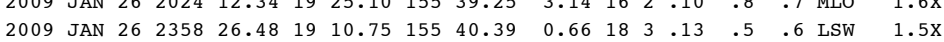

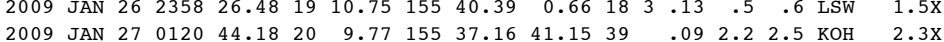

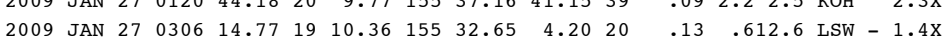

$\begin{array}{lllllllllllllllll}2009 & \text { JAN } 27 & 0436 & 42.16 & 19 & 28.28 & 154 & 56.15 & 1.74 & 20 & 1 & .13 & 2.0 & 2.2 & \text { SLE } & 1.6 \mathrm{X}\end{array}$ 2009 JAN $27 \quad \begin{array}{llllllllllllll}0520 & 41.13 & 19 & 25.64 & 155 & 37.95 & 3.25 & 17 & 3 & .07 & .4 & .5 & \text { MLO } & 1.6 \mathrm{X}\end{array}$

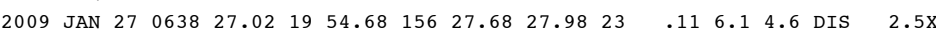

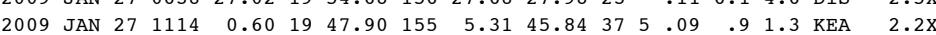

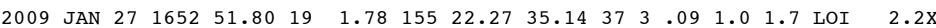

$\begin{array}{llllllllllllllll}2009 & \text { JAN } 28 & 0104 & 21.80 & 19 & 19.29 & 155 & 8.46 & 6.23 & 24 & 1 & .11 & .5 & 1.2 & \mathrm{SF} 4 & 1.2 \mathrm{X}\end{array}$

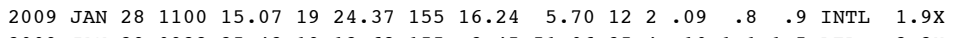
$\begin{array}{llllllllllllllll}2009 & \text { JAN } 29 & 0928 & 25.43 & 19 & 12.68 & 155 & 3.45 & 51.06 & 35 & 4 & .10 & 1.1 & 1.5 & \text { DEP } & 2.2 \mathrm{X}\end{array}$

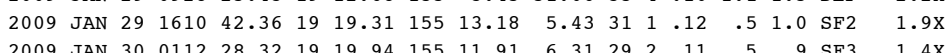

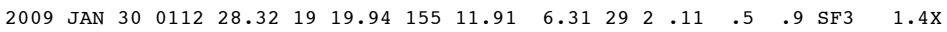

$\begin{array}{llllllllllllllll}2009 & \text { JAN } 30 & 0228 & 20.73 & 19 & 15.88 & 155 & 22.26 & 35.31 & 31 & 3 & .10 & .8 & 1.4 & \text { DEP } & 1.5 \mathrm{X}\end{array}$

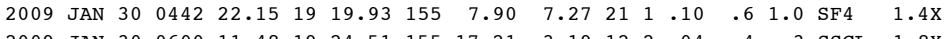

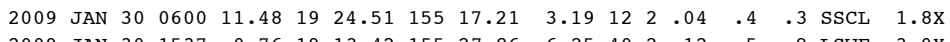

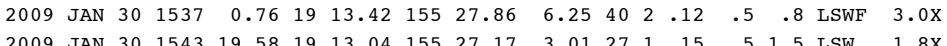

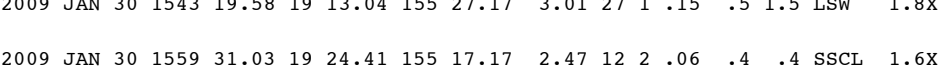

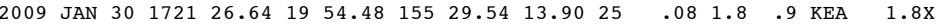

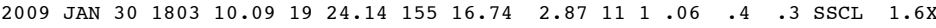

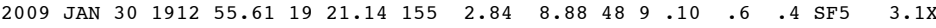

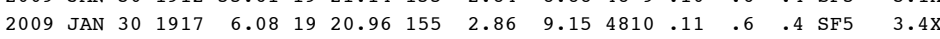

$\begin{array}{llllllllllllllll}2009 & \text { JAN } 30 & 2332 & 24.30 & 19 & 24.31 & 155 & 16.96 & 1.81 & 11 & 1 & .07 & .3 & .3 & \text { SSCL } & 1.7 \mathrm{x}\end{array}$

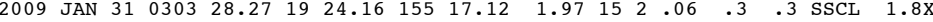

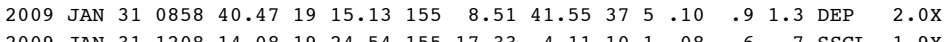
$\begin{array}{lllllllllllllllll}2009 & \text { JAN } 31 & 1208 & 14.08 & 19 & 24.54 & 155 & 17.33 & 4.11 & 10 & 1 & .08 & .6 & .7 & \text { SSCL } & 1.9 \mathrm{x} \\ \text { 2009 JAN } 31 & 2222 & 19.01 & 19 & 23.74 & 155 & 16.84 & 1.53 & 13 & 1 & .07 & .3 & .2 & \text { SSCL } & 1.7 \mathrm{x}\end{array}$

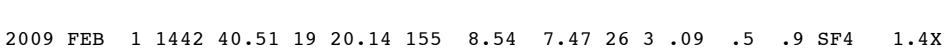

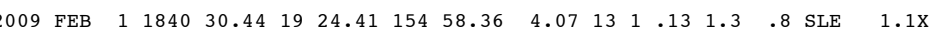
$\begin{array}{lllllllllllllllll}2009 & \text { FEB } & 1 & 2201 & 45.01 & 19 & 22.82 & 154 & 59.77 & 8.07 & 33 & 2 & .11 & 1.0 & .4 & \text { LER } & 1.9 \mathrm{X} \\ 2009 & \text { FEB } & 2 & 0314 & 3.66 & 19 & 24.26 & 155 & 17.54 & 3.15 & 11 & 1 & 09 & .5 & 4 & \text { SSCD } & 1.4 \mathrm{X}\end{array}$

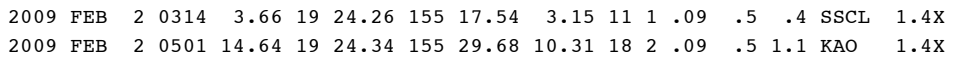

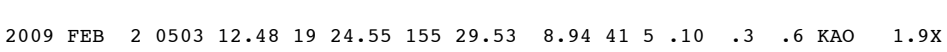
$\begin{array}{lllllllllllllllll}2009 & \text { FEB } & 2 & 0959 & 3.69 & 19 & 22.39 & 155 & 14.22 & 3.15 & 16 & 3 & .08 & .4 & .4 & \text { SEC } & 1.5 \mathrm{X}\end{array}$

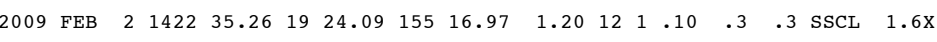
$\begin{array}{lllllllllllllllll}2009 & \text { FEB } & 2 & 1531 & 6.72 & 19 & 21.90 & 155 & 28.11 & 7.26 & 14 & 1 & .11 & .6 & 1.1 & \text { KAO } & 1.3 \mathrm{X}\end{array}$

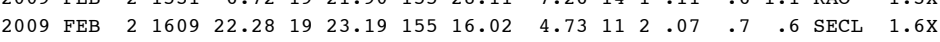

ORIGIN TIME (HST)-- -LAT N-- --LON W-- DEPTH N N RMS ERH ERZ LOC PREF N AZ MIN YEAR MON DA HRMN SEC DEG MIN DEG MIN KM RD S SEC KM KM REMKS MAG RD GAP DS

$\begin{array}{lllllllllllllllllll}2009 & \text { FEB } & 2 & 2348 & 33.84 & 19 & 23.99 & 155 & 16.86 & 2.20 & 12 & 2 & .09 & .4 & .3 & \text { SSCL } & 1.5 \mathrm{X} & 99 & 0\end{array}$

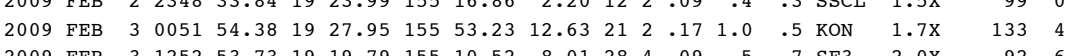

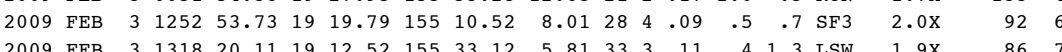

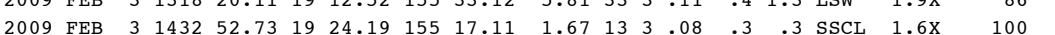

$\begin{array}{lllllllllllllllllll}2009 & \text { FEB } & 3 & 2119 & 13.31 & 19 & 29.67 & 155 & 26.03 & 8.15 & 28 & 4 & .13 & .4 & 1.0 & \text { KAO } & 1.5 \mathrm{X} & 91 & 5\end{array}$

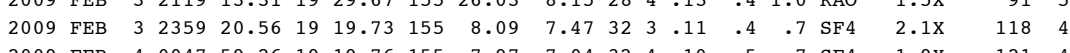

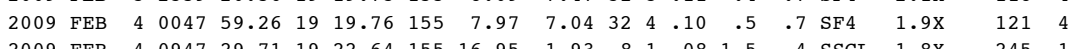

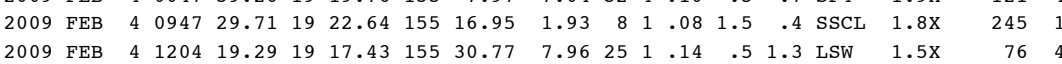

$\begin{array}{lllllllllllllllll}2009 & \text { FEB } & 4 & 1421 & 57.75 & 19 & 23.92 & 155 & 16.81 & 1.78 & 17 & 3 & .09 & .3 & .2 & \text { SSCL } & 1.8 \mathrm{X}\end{array}$ $\begin{array}{lllllllllllllllll}2009 & \text { FEB } & 4 & 1444 & 52.36 & 19 & 23.82 & 155 & 16.58 & 1.37 & 10 & 1 & .06 & .3 & .3 & \text { SSCL } & 1.2 \mathrm{X}\end{array}$

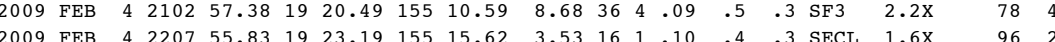
$\begin{array}{llllllllllllllllll}2009 & \text { FEB } & 4 & 2214 & 48.07 & 19 & 19.85 & 155 & 12.54 & 8.80 & 38 & 2 & .10 & .4 & .4 & \text { SF2 } & 1.9 \mathrm{X} & 79\end{array}$

$\begin{array}{lllllllllllllllll}2009 & \text { FEB } & 5 & 0236 & 26.25 & 19 & 18.77 & 155 & 29.08 & 3.51 & 27 & .08 & .4 & 4.1 & \text { LSW } & 1.3 \mathrm{X} & 63\end{array}$ $\begin{array}{lllllllllllllllllll}2009 & \text { FEB } & 5 & 0526 & 49.48 & 19 & 20.40 & 155 & 7.37 & 7.29 & 24 & 1 & .11 & .5 & .8 & \text { SF } 4 & 1.5 \mathrm{X} & 131 & 5\end{array}$

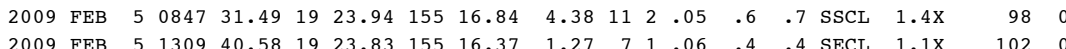

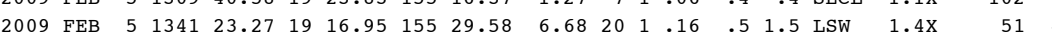

$\begin{array}{lllllllllllllllllll}2009 & \text { FEB } & 5 & 1552 & 44.79 & 19 & 24.52 & 155 & 16.65 & 1.76 & 15 & 2 & .07 & .4 & .2 & \text { SSCL } & 1.9 \mathrm{X} & 130 & 1\end{array}$

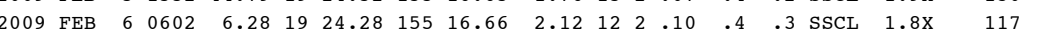

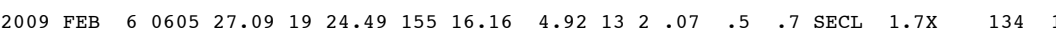

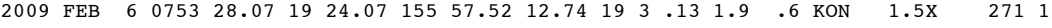

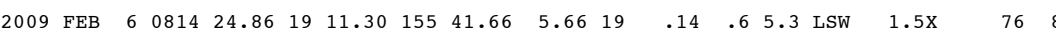

$\begin{array}{llllllllllllllllll}2009 & \text { FEB } & 6 & 0935 & 14.86 & 19 & 28.04 & 155 & 29.57 & 9.93 & 32 & 4 & .13 & .4 & 1.0 & \text { KAO } & 1.7 \mathrm{X} & 58\end{array}$

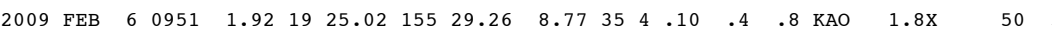

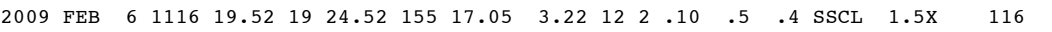

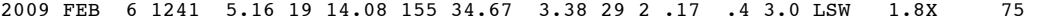

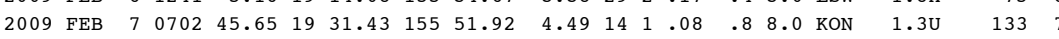

$\begin{array}{llllllllllllllllll}2009 & \text { FEB } & 7 & 0847 & 24.56 & 19 & 24.28 & 15 & 17.04 & 1.86 & 11 & 1 & .08 & .4 & .3 & \text { SSCL } & 1.2 \mathrm{X} & 106\end{array}$

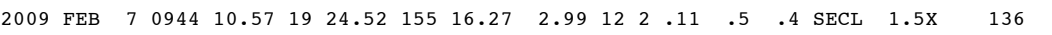

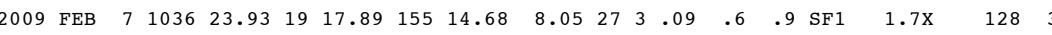

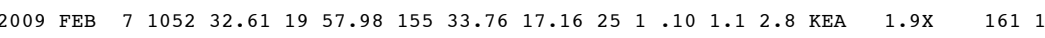

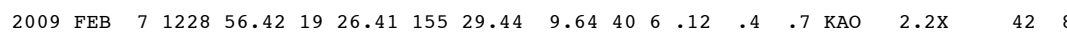

$\begin{array}{llllllllllllllllll}2009 & \text { FEB } & 7 & 1517 & 15.95 & 19 & 23.52 & 155 & 17.50 & 4.23 & 13 & 4 & .10 & .6 & .5 & \text { SSCL } & 1.8 \mathrm{X} & 149\end{array}$ $\begin{array}{lllllllllllllllllll}2009 & \text { FEB } & 7 & 1652 & 13.95 & 19 & 24.46 & 155 & 16.16 & 0.13 & 14 & 2 & .08 & .2 & .4 & \text { SECL } & 1.8 \mathrm{X} & 133\end{array}$

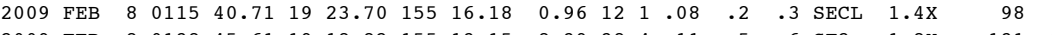

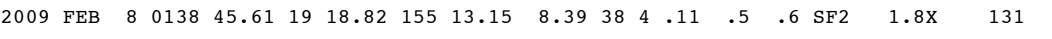
$\begin{array}{llllllllllllllllll}2009 & \text { FEB } & 8 & 0358 & 26.32 & 19 & 13.99 & 155 & 33.42 & 6.42 & 39 & 4 & .12 & .4 & 1.1 & \text { LSW } & 2.0 \mathrm{X} & 117\end{array}$

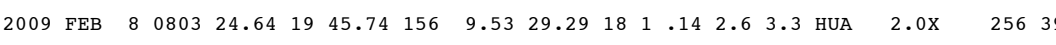

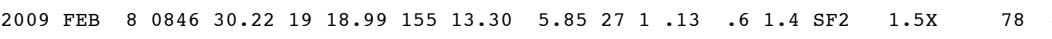
$\begin{array}{lllllllllllllllllll}2009 & \text { FEB } & 8 & 0957 & 40.79 & 19 & 24.82 & 155 & 17.03 & 3.57 & 14 & 4 & .09 & .4 & .3 & \text { SNCL } & 1.9 \mathrm{X} & 135\end{array}$

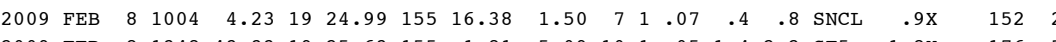
$\begin{array}{llllllllllllllllll}2009 & \text { FEB } & 8 & 1348 & 43.33 & 19 & 25.63 & 155 & 1.81 & 5.09 & 10 & 1 & .05 & 1.4 & 2.2 & \text { SF5 } & 1.8 \mathrm{X} & 176\end{array}$ 
---ORIGIN TIME (HST) -- -LAT N-- --LON W-- DEPTH N N RMS ERH ERZ LOC PREF N AZ MIN YEAR MON DA HRMN SEC DEG MIN DEG MIN KM RD S SEC KM KM REMKS MAG RD GAP DS

$\begin{array}{llllllllllllllll}2009 & \text { FEB } & 8 & 1936 & 34.24 & 19 & 20.18 & 155 & 10.82 & 8.05 & 27 & .08 & .4 & .7 & \text { SF } 3 & 1.8 \mathrm{X}\end{array}$

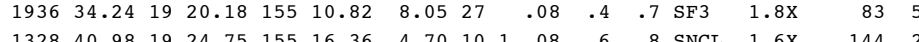

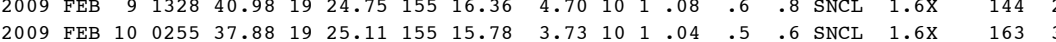

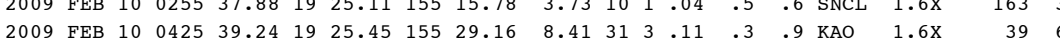

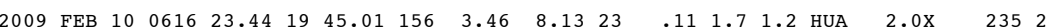

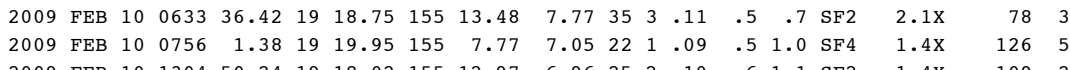

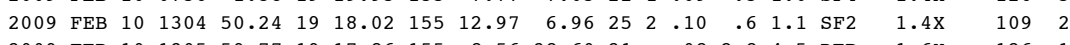

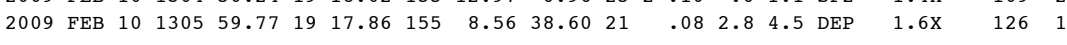

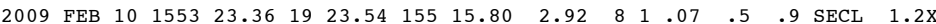

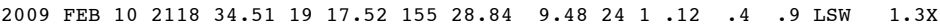

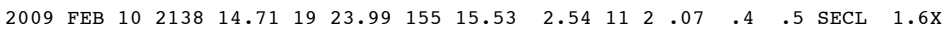

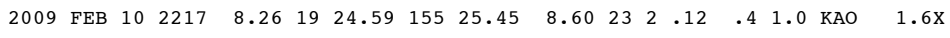

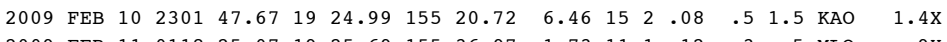

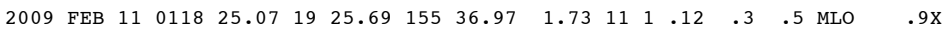

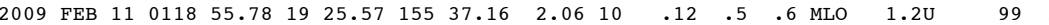

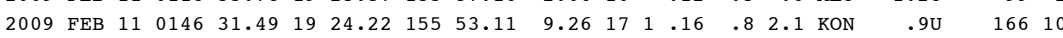

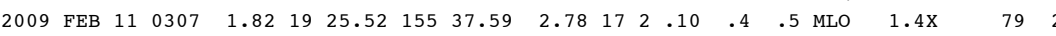

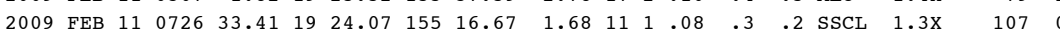

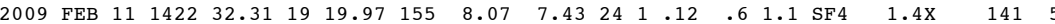

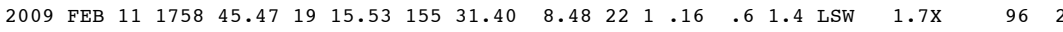

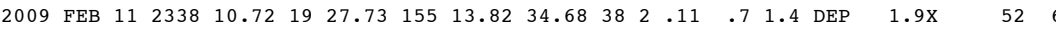

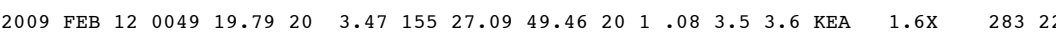

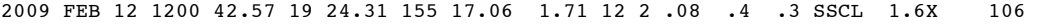

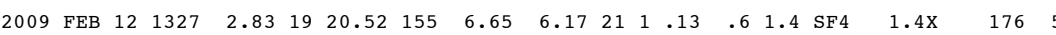

$\begin{array}{lllllllllllllllll}2009 & \text { FEB } 12 & 1718 & 31.71 & 19 & 11.60 & 155 & 24.83 & 34.93 & 26 & 1 & .11 & 1.1 & 2.0 & \text { DEP } & 1.7 \mathrm{X} & 165\end{array}$

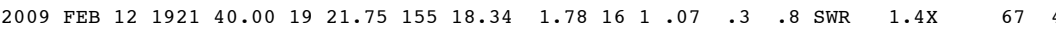

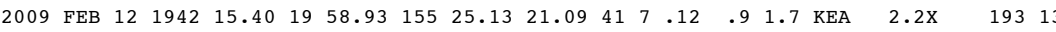

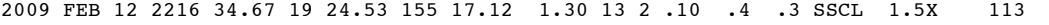

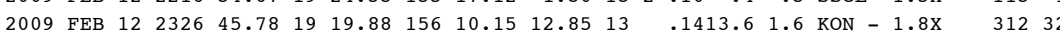

$\begin{array}{lllllllllllllllllll}2009 & \text { FEB } & 12 & 2341 & 13.85 & 19 & 23.78 & 155 & 16.88 & 1.93 & 12 & 1 & .09 & .4 & .3 & \text { SSCL } & 1.7 \mathrm{X} & 87 & 1\end{array}$

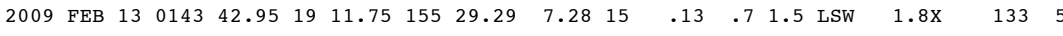

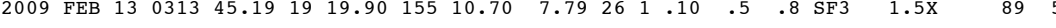

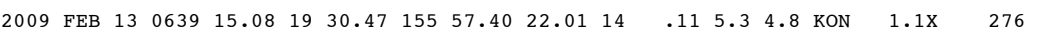
2009 FEB $13 \quad 0945 \quad 44.6519 \quad 30.50 \quad 155 \quad 50.91 \quad 8.00 \quad 13 \quad 1.11 \quad 1.1 \quad 1.6$ KON $\quad 1.1 \mathrm{x} \quad 180 \quad 8$

2009 FEB $13121520.991928 .40156 \quad 29.59 \quad 38.04 \quad 15 \quad 1.12 \quad 4.7 \quad 4.2$ DIS $2.3 \times \quad 32360$

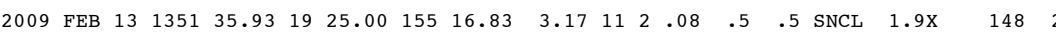

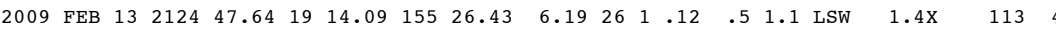

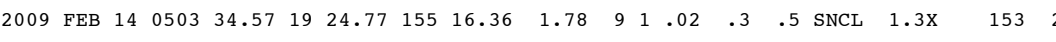

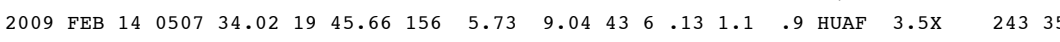

$\begin{array}{lllllllllllllllllll}2009 & \text { FEB } & 14 & 0522 & 9.96 & 19 & 24.46 & 155 & 31.82 & 45.74 & 30 & 5 & .09 & .7 & 1.2 & \text { DMLF } & 2.2 \mathrm{X} & 49 & 2\end{array}$

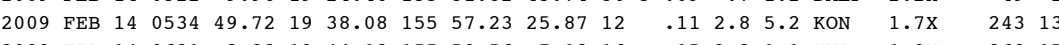

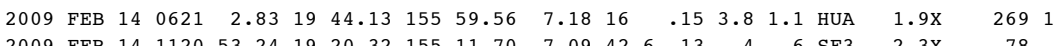

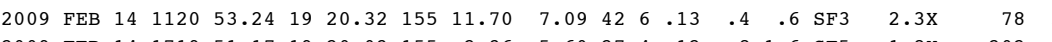

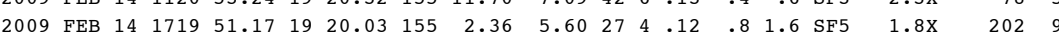

---ORIGIN TIME (HST)-- -LAT N-- --LON W-- DEPTH N N RMS ERH ERZ LOC PREF N AZ MIN YEAR MON DA HRMN SEC DEG MIN DEG MIN KM RD S SEC KM KM REMKS MAG RD GAP DS

$\begin{array}{lllllllllllllllllll}2009 & \text { FEB } & 14 & 1843 & 23.92 & 19 & 24.15 & 155 & 17.05 & 1.50 & 13 & 2 & .06 & .3 & .2 & \text { SSCL } & 1.6 \mathrm{X} & 100 & 1\end{array}$

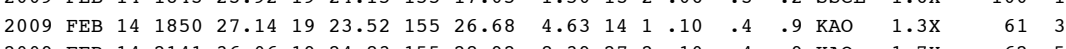

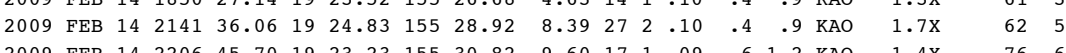

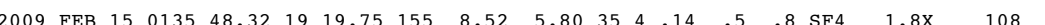

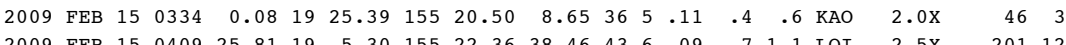

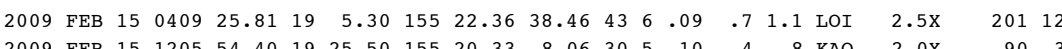

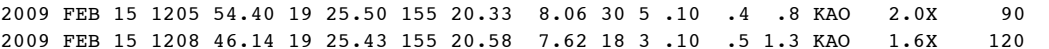

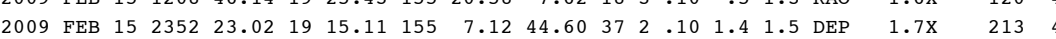

2009 FEB $16 \quad 0245 \quad 19.54 \quad 1955.44 \quad 156 \quad 10.05 \quad 35.34 \quad 48 \quad 9.11 \quad 1.2 \quad 1.7$ кон $3.2 \mathrm{X} \quad 264 \quad 43$

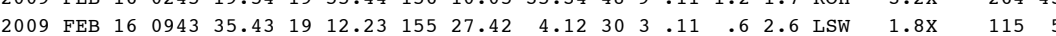

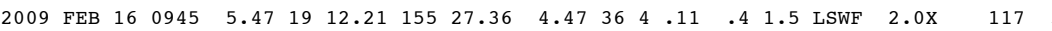

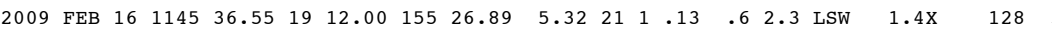

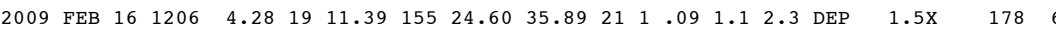

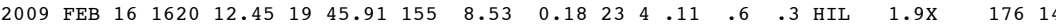

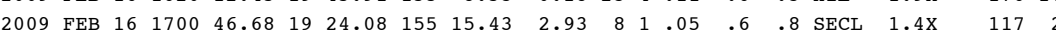

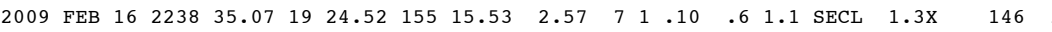

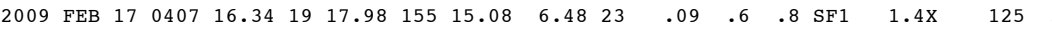

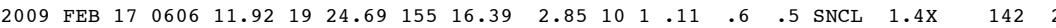

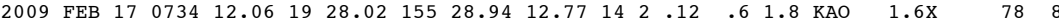

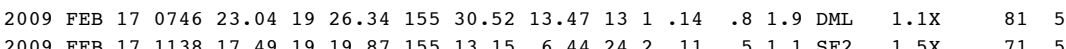

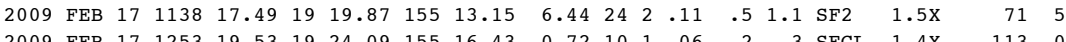

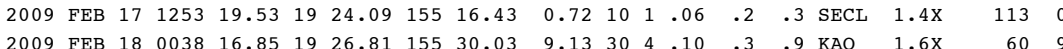

$\begin{array}{lllllllllllllllll}2009 & \text { FEB } 18 & 0127 & 41.96 & 19 & 26.76 & 155 & 51.76 & 15.63 & 13 & 1 & .14 & 1.4 & 1.5 & \text { KON } & .9 \mathrm{X} & 126\end{array}$

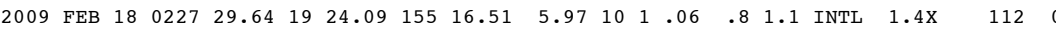

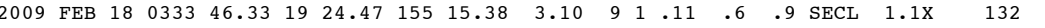

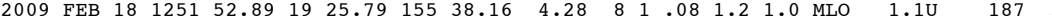

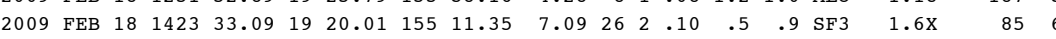

$\begin{array}{lllllllllllllllllll}2009 & \text { FEB } & 18 & 1839 & 28.27 & 19 & 24.27 & 155 & 14.79 & 6.76 & 7 & 1 & .06 & 1.4 & 3.4 & \text { INTL } & 1.3 \mathrm{X} & 113\end{array}$

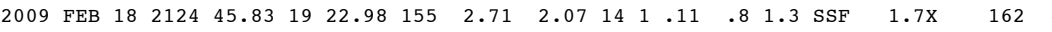
$\begin{array}{llllllllllllllllll}2009 & \text { FEB } & 18 & 2130 & 49.49 & 19 & 19.29 & 155 & 24.11 & 31.65 & 23 & 2 & .08 & .7 & 1.5 & \text { DEP } & 1.1 X & 75\end{array}$

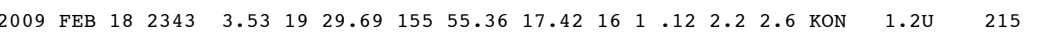

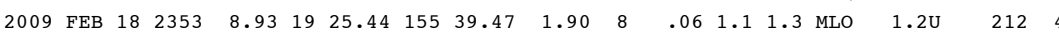

$\begin{array}{llllllllllllllllll}2009 & \text { FEB } 19 & 0120 & 16.96 & 19 & 23.46 & 155 & 16.71 & 2.83 & 13 & 1 & .06 & .4 & .3 & \text { SSCL } & 1.9 \mathrm{X} & 99 & 0\end{array}$

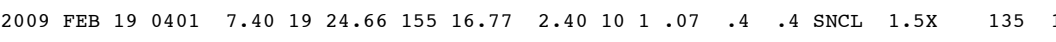

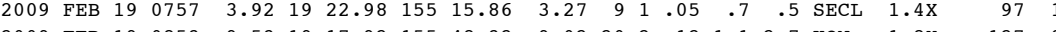

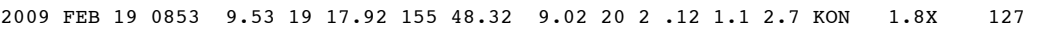

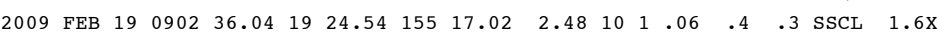

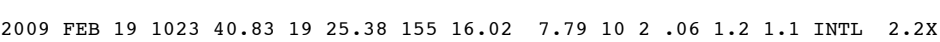

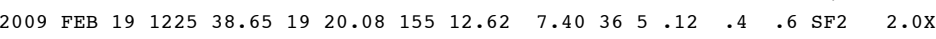

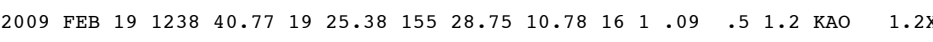

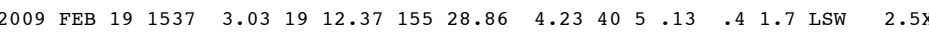

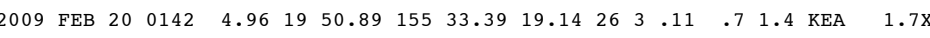


---ORIGIN TIME (HST)-- -LAT N-- --LON W-- DEPTH N N RMS ERH ERZ LOC PREF N AZ MIN YEAR MON DA HRMN SEC DEG MIN DEG MIN KM RD S SEC KM KM REMKS MAG RD GAP DS

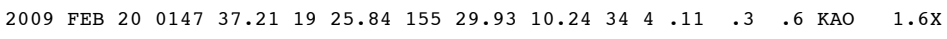
$\begin{array}{llllllllll}2009 & & \end{array}$

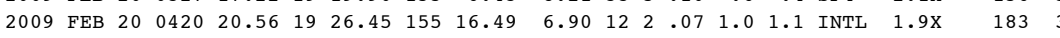

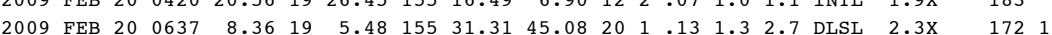

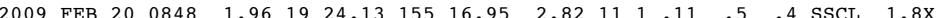

$\begin{array}{llllllllllllllllll}2009 & \text { FEB } 20 & 1219 & 35.31 & 19 & 24.69 & 155 & 16.76 & 4.56 & 10 & 1 & .07 & .7 & .7 & \text { SNCL } & 1.7 \mathrm{X} & 138 & 1\end{array}$

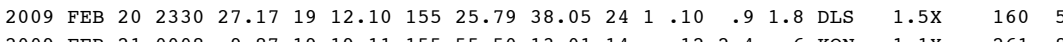

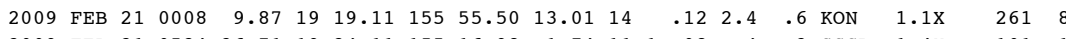

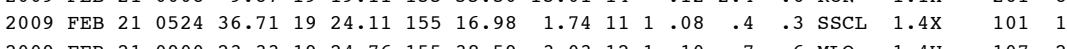
$\begin{array}{lllllllllllllllll}2009 & \text { FEB } & 21 & 0900 & 23.33 & 19 & 24.76 & 155 & 38.59 & 3.03 & 12 & 1 & .10 & .7 & .6 & \text { MLO } & 1.4 \mathrm{U}\end{array}$

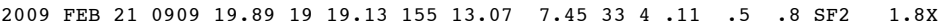

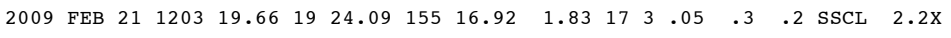

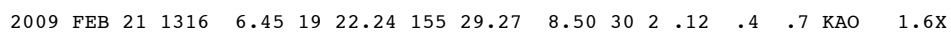

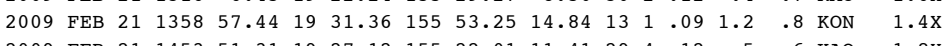

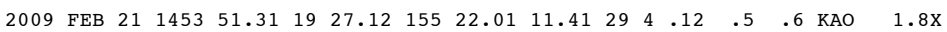

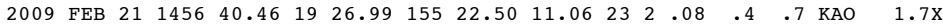

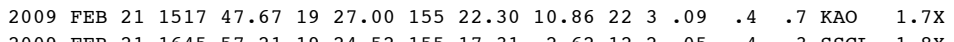

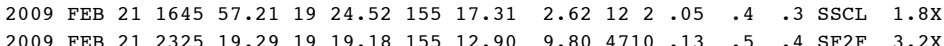

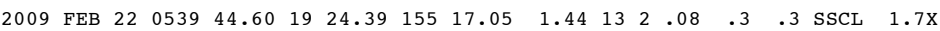

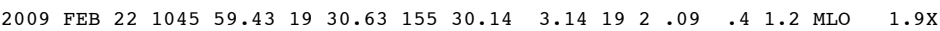

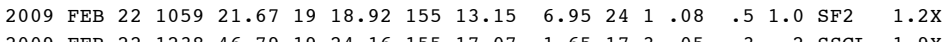

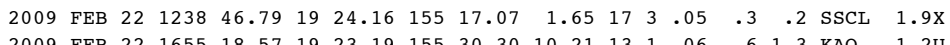

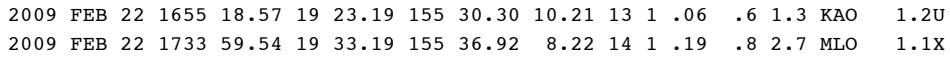

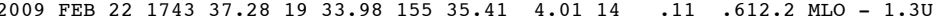

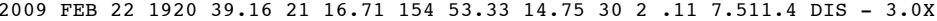

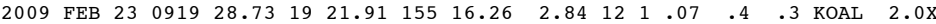

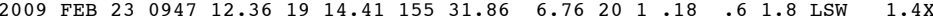

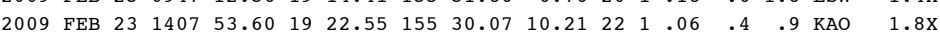

$\begin{array}{lllllllllllllll}2009 & \text { FEB } & 23 & 1409 & 2.29 & 19 & 24.71 & 155 & 26.84 & 10.22 & 23 & 1 & .11 & .4 & .9 \\ \text { KAO } & 1.6 \mathrm{X}\end{array}$

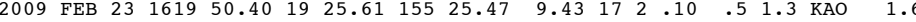

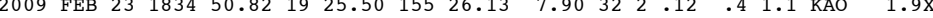

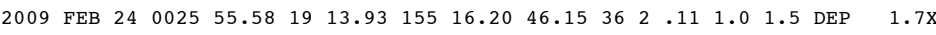

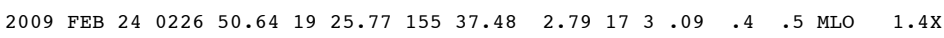

$\begin{array}{llllllllllllllll}2009 & \text { FEB } 24 & 0521 & 6.95 & 19 & 20.01 & 155 & 5.13 & 8.84 & 24 & 1 & .11 & .7 & .5 & \text { SF5 } & 1.6 \mathrm{X}\end{array}$

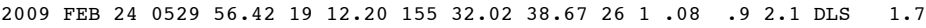

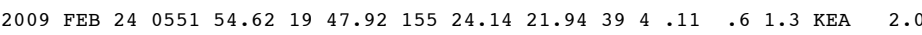

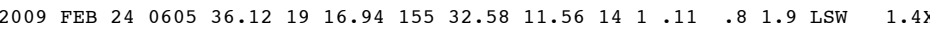

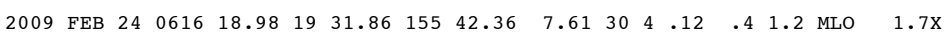

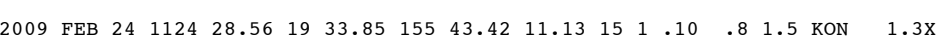

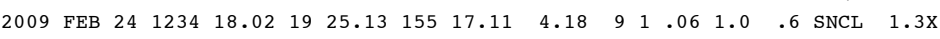

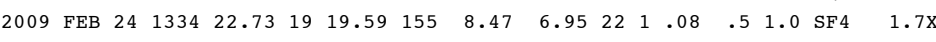

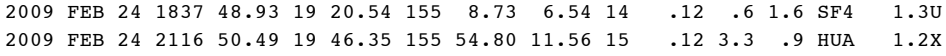

---ORIGIN TIME (HST)-- -LAT N-- --LON W-- DEPTH N N RMS ERH ERZ LOC PREF N AZ MIN YEAR MON DA HRMN SEC DEG MIN DEG MIN KM RD S SEC KM KM REMKS MAG RD GAP DS

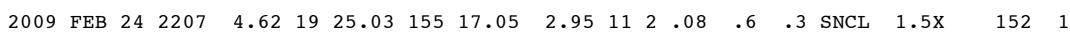

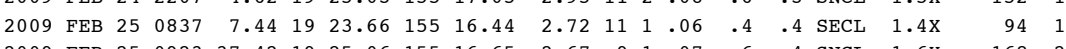

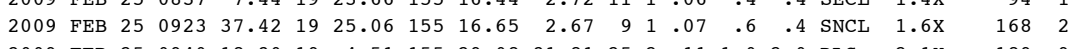

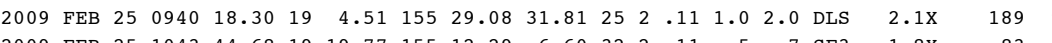

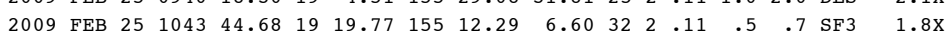

$\begin{array}{lllllllllllllll}2009 & \text { FEB } 25 & 1543 & 57.14 & 19 & 20.38 & 155 & 3.91 & 4.29 & 19 & .12 & .9 & 5.9 & \text { SSF } & 1.7 \mathrm{X}\end{array}$

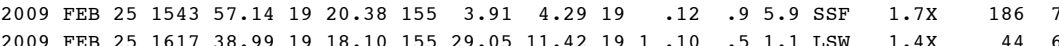

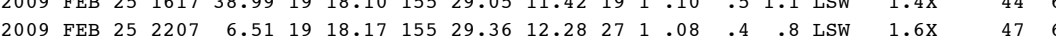

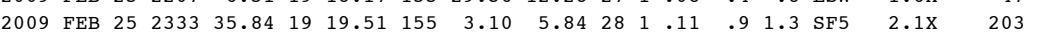

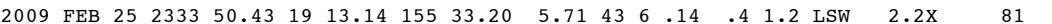

$\begin{array}{lllllllllllllllll}2009 & \text { FEB } 25 & 2335 & 47.98 & 19 & 19.12 & 155 & 2.91 & 4.51 & 24 & 1 & .10 & 1.1 & 3.0 & \text { SSF } & 1.8 \mathrm{X} & 210\end{array}$

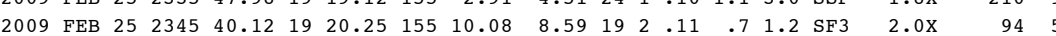

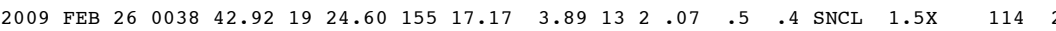

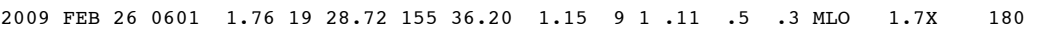

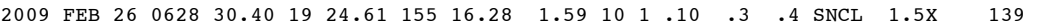

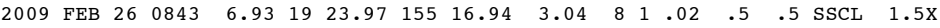

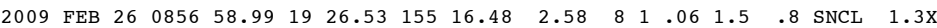

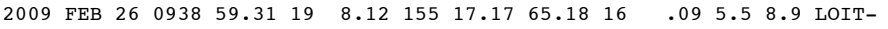

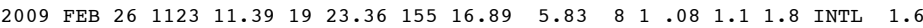

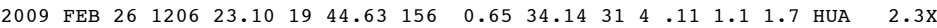

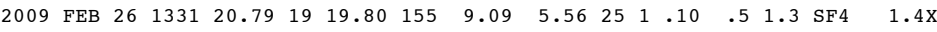

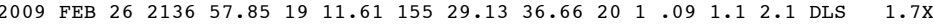

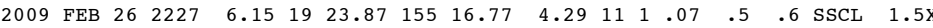

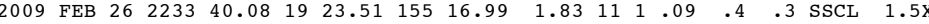
$\begin{array}{llllllllllllll}2009 & \text { FEB } & 26 & 2253 & 7.86 & 19 & 52.44 & 155 & 49.36 & 16.05 & 10 & 1.16 & 1.2 & 1.5\end{array}$

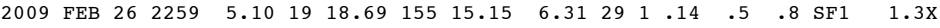

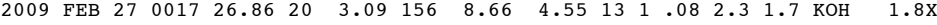

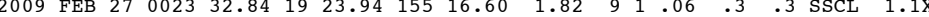

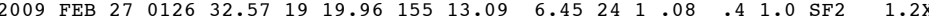

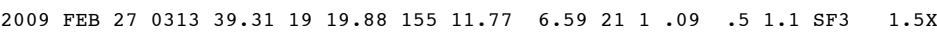

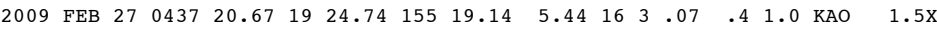

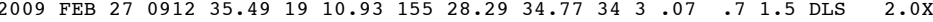

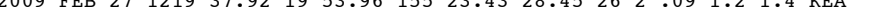

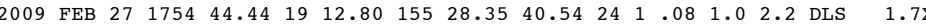

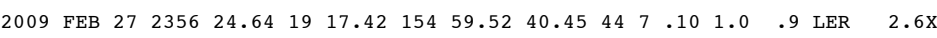

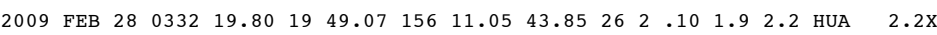

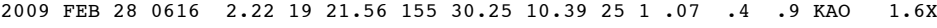

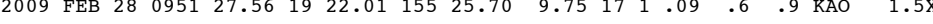

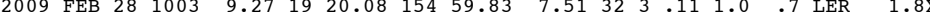

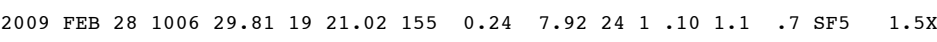

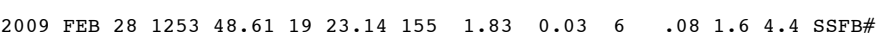

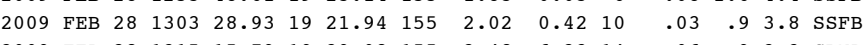

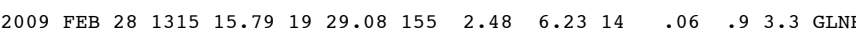

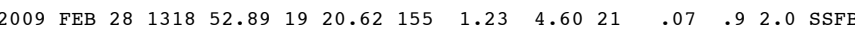

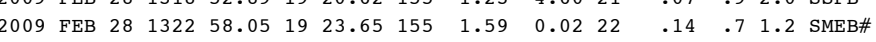

$96 \quad 1$ 23517 $143 \quad 0$ 22519

$95 \quad 5$ 97
97 82 $184 \quad 17$

$97 \quad 4$ $276 \quad 39$ 103 $\begin{array}{ll}70 & 5 \\ 86 & 6\end{array}$

102 93 $217 \quad 5$ $99 \quad 5$ 23314

29346 $\begin{array}{ll}53 & 5 \\ 69 & 3\end{array}$ $\begin{array}{rr}69 & 3 \\ 221 & 10\end{array}$ 2088

$171 \quad 5$ $\begin{array}{rr}182 & 6 \\ 176 & 10\end{array}$ $176 \quad 10$ $204 \quad 9$ 
---ORIGIN TIME (HST)-- -LAT N-- --LON W-- DEPTH N N RMS ERH ERZ LOC PREF N AZ MIN YEAR MON DA HRMN SEC DEG MIN DEG MIN KM RD S SEC KM KM REMKS MAG RD GAP DS

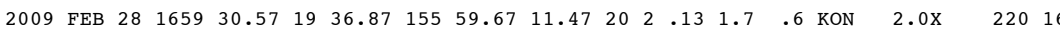

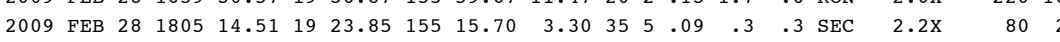

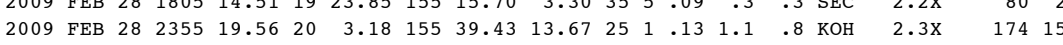

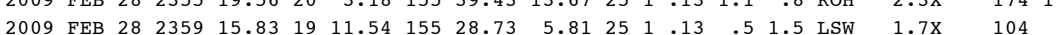

$\begin{array}{lllllllllllllllllll}2009 & \text { MAR } & 1 & 0059 & 28.81 & 19 & 25.97 & 155 & 29.53 & 11.00 & 25 & 2 & .09 & .4 & .8 & \text { KAO } & 1.4 \mathrm{X} & 63 & 7\end{array}$

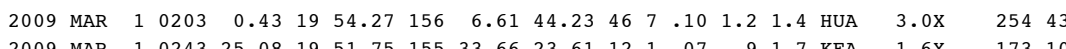

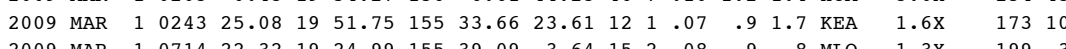

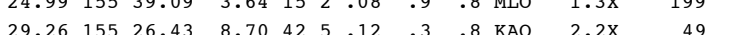

$\begin{array}{llllllllllllllllll}2009 & \text { MAR } & 1 & 1505 & 43.30 & 19 & 24.40 & 155 & 17.04 & 1.93 & 10 & 1 & .03 & .3 & .3 & \text { SSCL } & 1.7 \mathrm{X} & 110\end{array}$

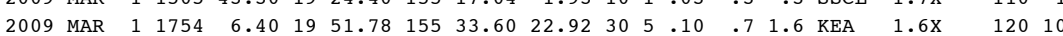

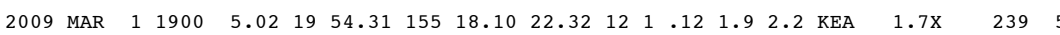

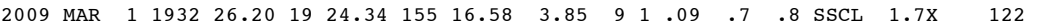

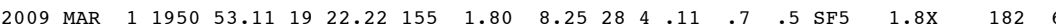

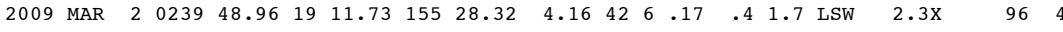

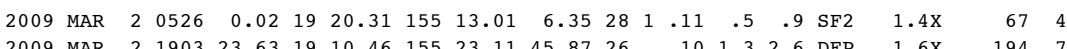

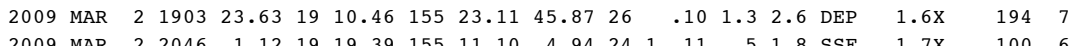

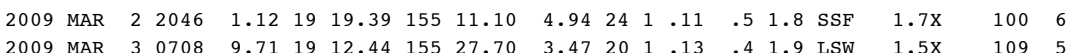

$\begin{array}{llllllllllllllllll}2009 & \text { MAR } & 3 & 1209 & 55.14 & 19 & 18.31 & 155 & 15.70 & 4.78 & 19 & .10 & .7 & 2.5 & \text { SSF } & 1.5 \mathrm{X} & 123 & 5\end{array}$

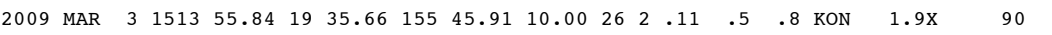

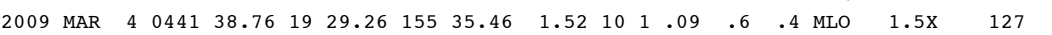

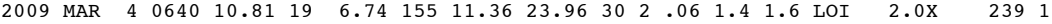

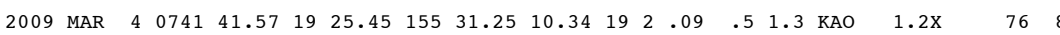

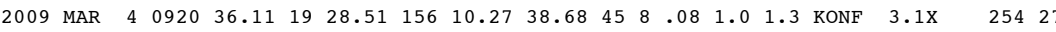

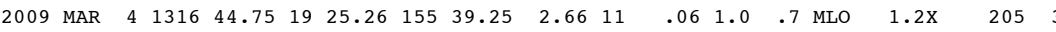

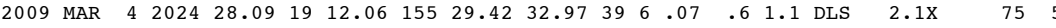

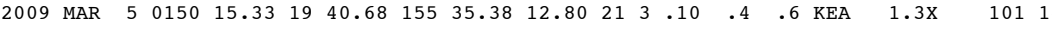
$\begin{array}{llllllllllllllllll}2009 & \text { MAR } & 5 & 0825 & 57.94 & 19 & 28.55 & 154 & 52.56 & 0.28 & 27 & 3 & .16 & 1.1 & .4 & \text { SLEF } & 2.5 \mathrm{X} & 265\end{array}$

$\begin{array}{lllllllllllllllllll}2009 & \text { MAR } & 5 & 1227 & 15.29 & 19 & 19.85 & 155 & 8.27 & 7.03 & 26 & 2 & .11 & .5 & .9 & \mathrm{SF} 4 & 1.8 \mathrm{X} & 114 & 5\end{array}$

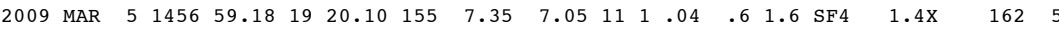

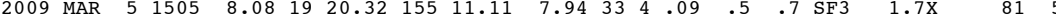

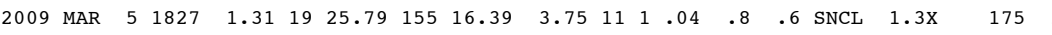

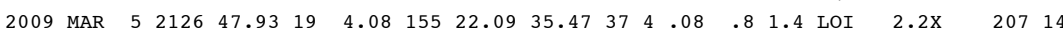

2009 MAR $55225142.01 \quad 1953.8515625 .8018 .34201 \quad .113 .312 .4$ DIS $-1.8 \mathrm{X} 31866$ $\begin{array}{lllllllllllllllll}2009 \text { MAR } & 5 & 2302 & 39.53 & 19 & 20.22 & 155 & 6.75 & 5.05 & 33 & 3 & .12 & .5 & 1.1 & \text { SF4 } & 1.8 \mathrm{X} & 145\end{array}$

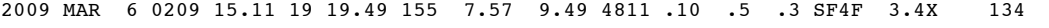
2009 MAR $6 \begin{array}{lllllllllllllllll}1054 & 17.08 & 19 & 22.19 & 155 & 27.70 & 9.43 & 38 & 4 & .11 & .4 & .6 & \text { KAO } & 2.0 \mathrm{X} & 40\end{array}$ $\begin{array}{llllllllllllllllll}2009 \text { MAR } & 6 & 1549 & 24.77 & 19 & 24.58 & 155 & 38.56 & 3.09 & 14 & 2 & .10 & .8 & .5 & \text { MLO } & 1.5 \mathrm{x} & 183 & 1\end{array}$

$\begin{array}{lllllllllllllllllll}2009 & \text { MAR } & 6 & 1604 & 46.68 & 19 & 19.61 & 155 & 7.85 & 8.47 & 31 & 3 & .09 & .5 & .6 & \text { SF } 4 & 2.0 \mathrm{X} & 126 & 4\end{array}$

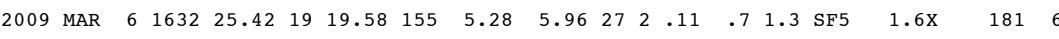

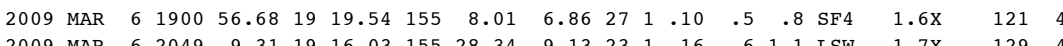

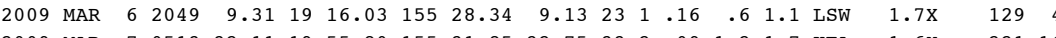

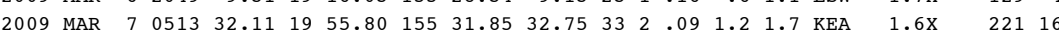

---ORIGIN TIME (HST)-- -LAT N-- --LON W-- DEPTH N N RMS ERH ERZ LOC PREF N AZ MIN YEAR MON DA HRMN SEC DEG MIN DEG MIN KM RD S SEC KM KM REMKS MAG RD GAP DS

$\begin{array}{lllllllllllllllllll}2009 & \text { MAR } & 7 & 0628 & 51.82 & 19 & 25.17 & 155 & 16.51 & 3.05 & 10 & 1 & .03 & .6 & .5 & \text { SNCL } & 1.7 \mathrm{X} & 157 & 2\end{array}$

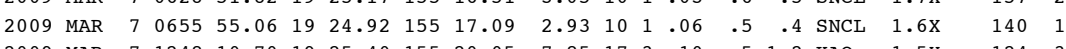

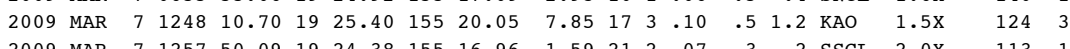
$\begin{array}{lllllllllllllll}2009 & \text { MAR } & 7 & 1412 & 42.39 & 19 & 24.78 & 155 & 16.54 & 2.02 & 10 & 1 & 05 & 4 & 4\end{array}$

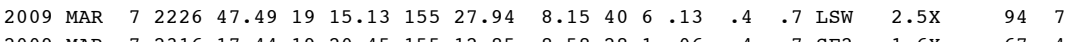
$\begin{array}{lllllllllllllllllll}2009 & \text { MAR } & 7 & 2316 & 17.44 & 19 & 20.45 & 155 & 12.85 & 8.58 & 28 & 1 & .06 & .4 & .7 & \text { SF2 } & 1.6 \mathrm{X} & 67 & 4\end{array}$

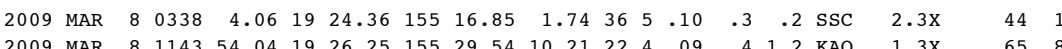
2009 MAR $8 \begin{array}{lllllllllllllll}1143 & 54.04 & 19 & 26.25 & 155 & 29.54 & 10.21 & 22 & 4 & .09 & .4 & 1.2 & \text { KAO } & 1.3 \mathrm{X} & 65 \\ 8\end{array}$

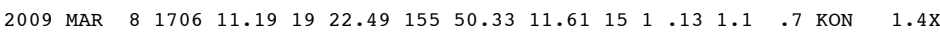

$\begin{array}{lllllllllllllllll}2009 & \text { MAR } & 8 & 2242 & 14.51 & 19 & 24.71 & 155 & 16.94 & 3.68 & 12 & 2 & .07 & .5 & .5 & \text { SNCL } & 1.7 \mathrm{X}\end{array}$

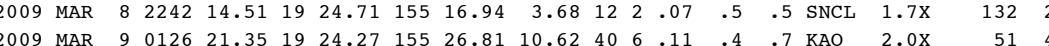

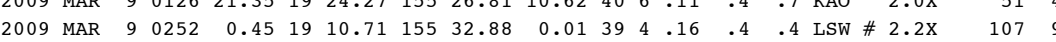
$\begin{array}{lllllllllllllllllll}2009 & \text { MAR } & 9 & 0944 & 53.14 & 20 & 8.30 & 15 & 9.90 & 23.31 & 47 & 8 & .11 & 1.2 & 3.3 & \mathrm{KEAF} & 4.3 \mathrm{X} & 251 & 33\end{array}$ $\begin{array}{llllllllllllllll}2009 & \text { MAR } & 9 & 1119 & 12.73 & 19 & 25.11 & 15 & 17.14 & 4.98 & 11 & 1.08 & .7 & .7 & \text { SNCL } & 1.7 \mathrm{X}\end{array}$

$\begin{array}{lllllllllllllllll}2009 & \text { MAR } & 9 & 1222 & 24.55 & 19 & 14.86 & 155 & 36.87 & 2.57 & 24 & 1 & .13 & .6 & 2.5 & \text { LSW } & 1.8 \mathrm{x}\end{array}$

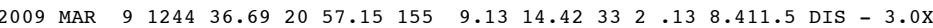

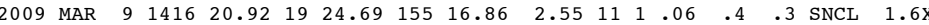

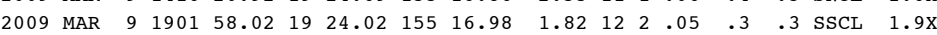

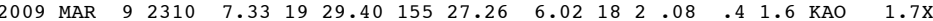

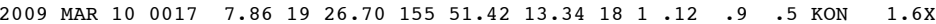

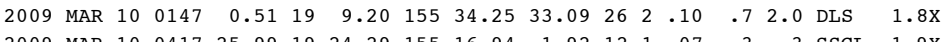

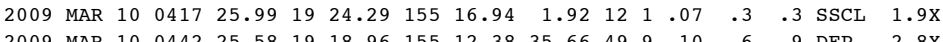

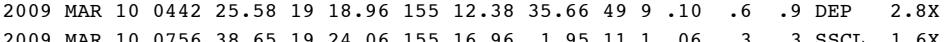

$\begin{array}{llllllllllllllll}2009 \text { MAR } & 10 & 1146 & 32.61 & 19 & 15.12 & 155 & 34.22 & 8.48 & 21 & 1 & .13 & .5 & 1.4 & \text { LSW } & 1.8 x\end{array}$ 2009 MAR $1011559 \begin{array}{lllllllllllllll}54.81 & 19 & 19.47 & 155 & 7.63 & 8.65 & 29 & 4 & .07 & .5 & .7 & \mathrm{SF} 4 & 2.0 \mathrm{X}\end{array}$

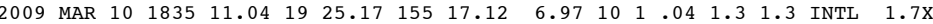
2009 MAR $10 \begin{array}{lllllllllllllll}2138 & 43.61 & 19 & 26.90 & 155 & 16.96 & 7.39 & 10 & 1 & .05 & 2.3 & 1.3 & \text { INTL } & 2.0 \mathrm{X}\end{array}$

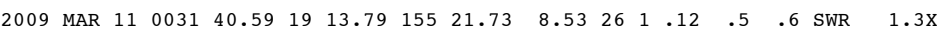

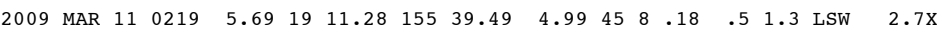

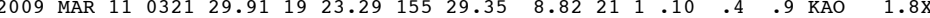

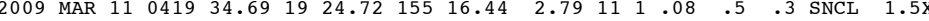

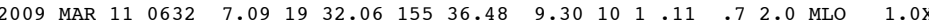

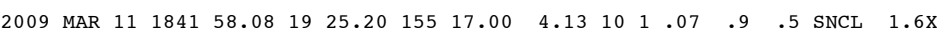

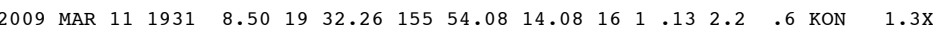

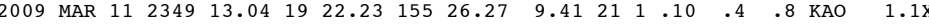

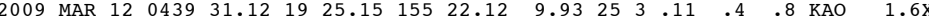

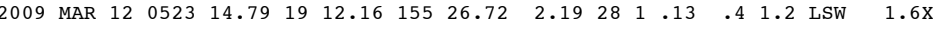

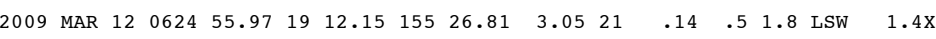

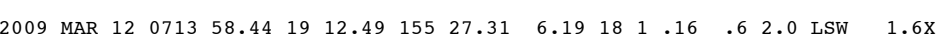

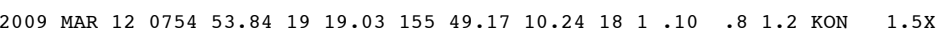

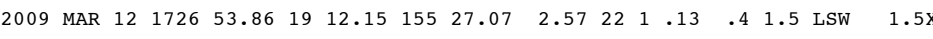

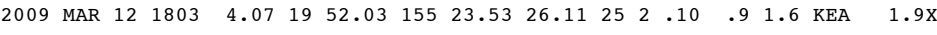

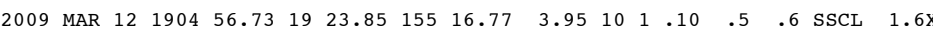


---ORIGIN TIME (HST)-- -LAT N-- --LON W-- DEPTH N N RMS ERH ERZ LOC PREF N AZ MIN YEAR MON DA HRMN SEC DEG MIN DEG MIN KM RD S SEC KM KM REMKS MAG RD GAP DS

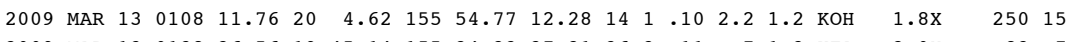

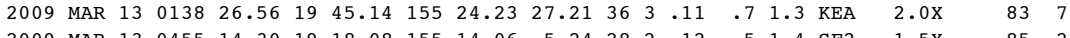

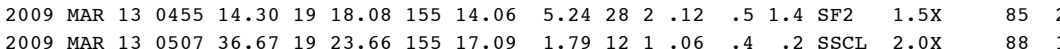

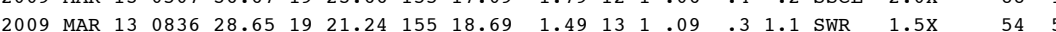

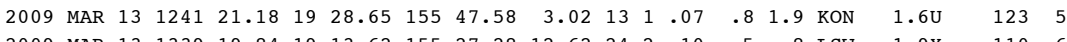

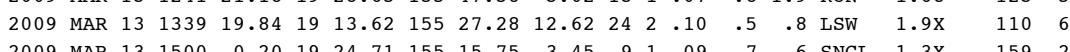

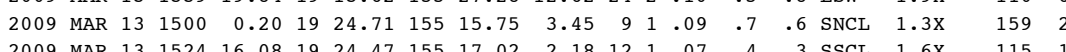

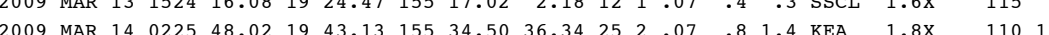

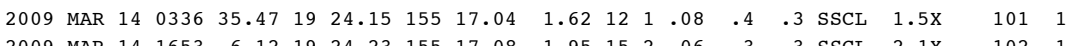

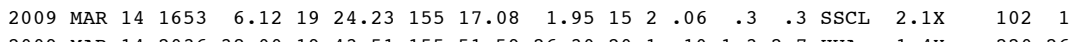

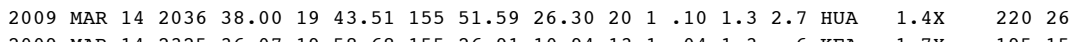

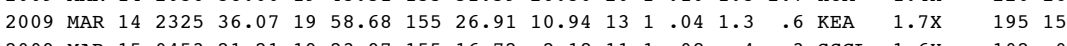

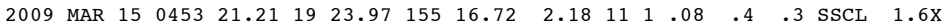

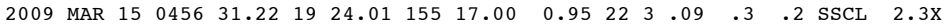

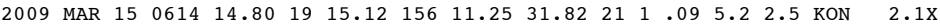

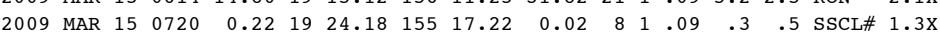

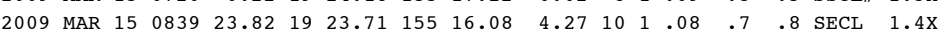

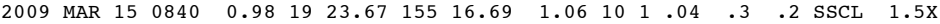

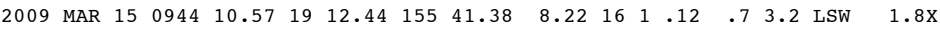

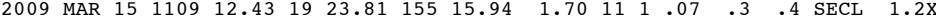

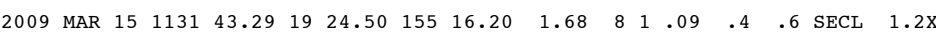

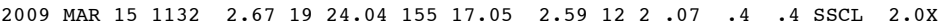

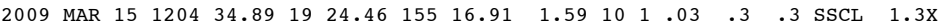

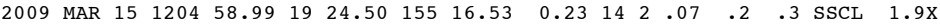

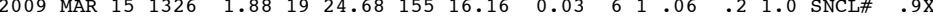

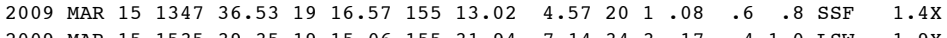

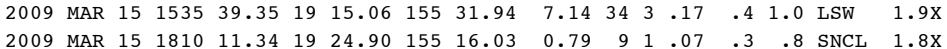

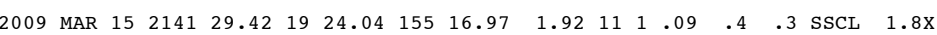

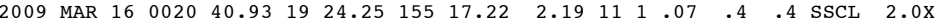

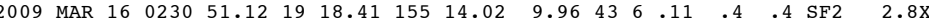

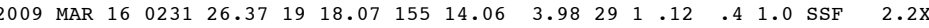

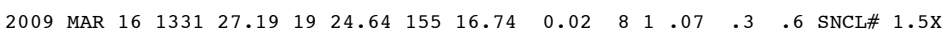

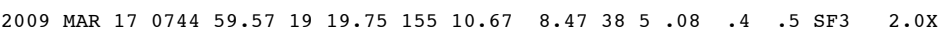
2009 MAR $17 \begin{array}{llllllllllllll}1415 & 33.29 & 19 & 23.15 & 155 & 17.31 & 3.28 & 11 & 1 & .06 & .5 & .5 & \text { SSCL } & 1.8 \mathrm{x}\end{array}$

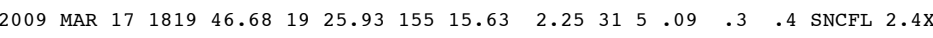

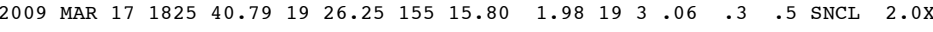
2009 MAR $17 \quad 1959 \begin{array}{lllllllllllllll}38.55 & 19 & 25.14 & 155 & 16.38 & 3.66 & 10 & 1 & .10 & .7 & .6 & \text { SNCL } & 1.7 \mathrm{X}\end{array}$

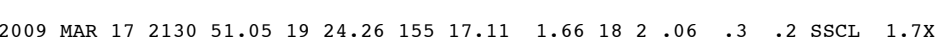

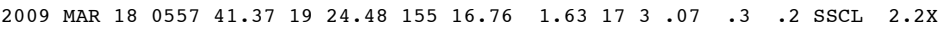

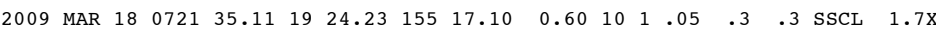

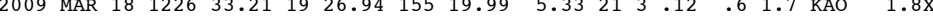

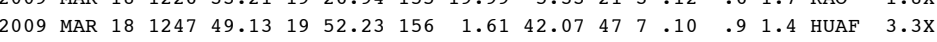

---ORIGIN TIME (HST)-- -LAT N-- --LON W-- DEPTH N N RMS ERH ERZ LOC PREF N AZ MIN YEAR MON DA HRMN SEC DEG MIN DEG MIN KM RD S SEC KM KM REMKS MAG RD GAP DS

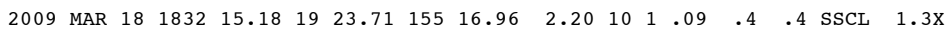

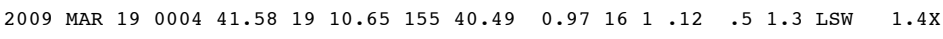

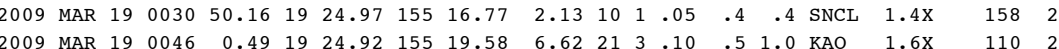

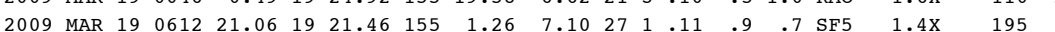

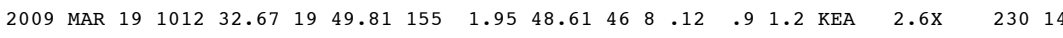

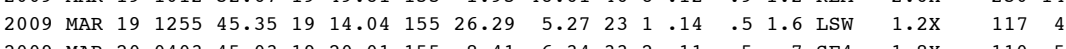

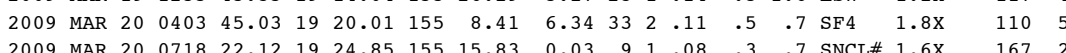

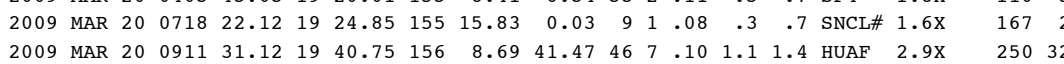

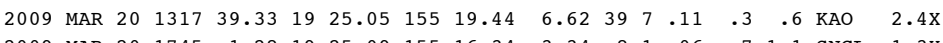

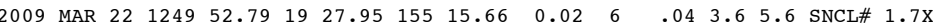

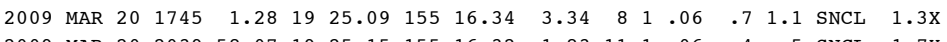

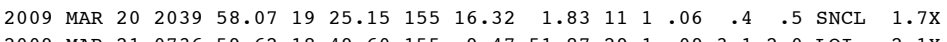

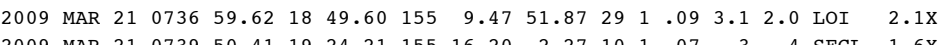

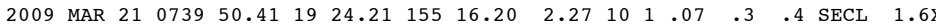

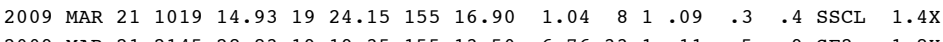

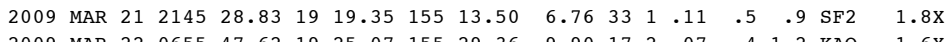
$\begin{array}{lllllllllllllll}2009 \text { MAR } 22 & 0655 & 47.62 & 19 & 25.07 & 155 & 29.36 & 9.90 & 17 & 2 & .07 & .4 & 1.2 & \text { KAO } & 1.6 \mathrm{X}\end{array}$

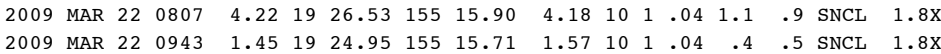
$\begin{array}{llllllllllllllll}2009 & \text { MAR } 22 & 1239 & 34.24 & 19 & 23.98 & 155 & 15.79 & 0.21 & 10 & 1 & .05 & .2 & .4 & \text { SECL } & 1.6 \mathrm{X}\end{array}$

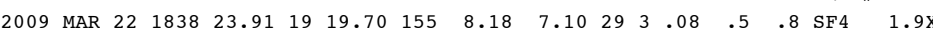

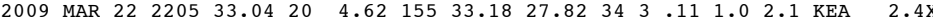

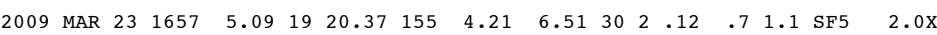

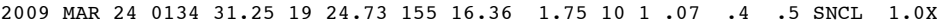

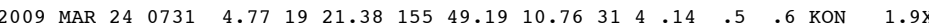

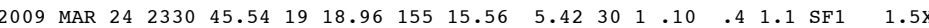

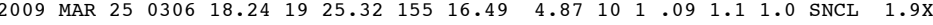

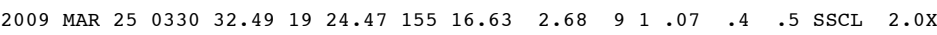

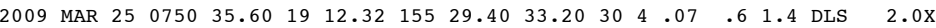

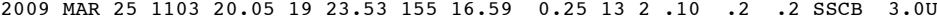

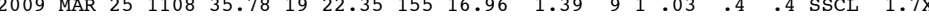

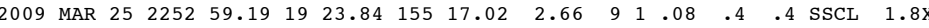

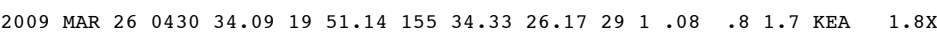

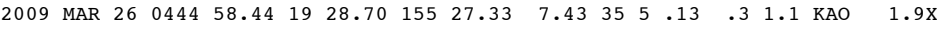

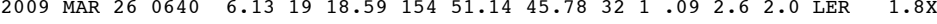

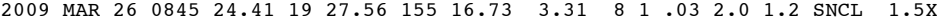

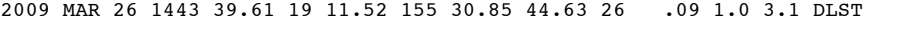

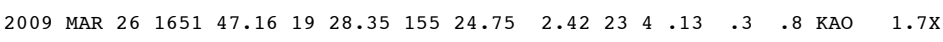

$\begin{array}{llllllllllllll}2009 & \text { MAR } & 26 & 1755 & 47.39 & 19 & 25.26 & 155 & 39.60 & 0.80 & 8 & .15 & 1.0 & 2.1 \\ \text { MLO } & 1.2 \mathrm{X}\end{array}$

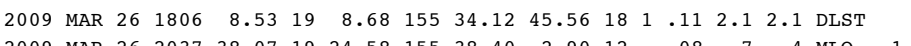

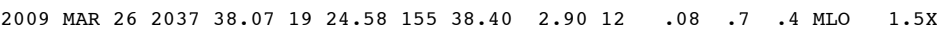

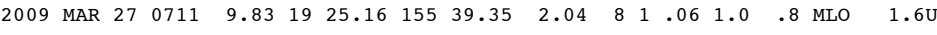

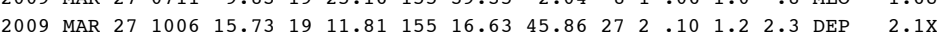

$46 \quad 3$

177

157

123

$105 \quad 1$

$\begin{array}{rr}69 & 4 \\ 63 & 6\end{array}$

63

$177 \quad 2$

113 116 20324

1807

151 11611 128 
---ORIGIN TIME (HST)-- -LAT N-- --LON W-- DEPTH N N RMS ERH ERZ LOC PREF N AZ MIN YEAR MON DA HRMN SEC DEG MIN DEG MIN KM RD S SEC KM KM REMKS MAG RD GAP DS

$\begin{array}{llllllllllllllll}2009 & \text { MAR } 27 & 1018 & 25.68 & 19 & 15.64 & 155 & 19.78 & 43.72 & 18 & .11 & 1.7 & 4.0 & \text { DEPT }\end{array}$

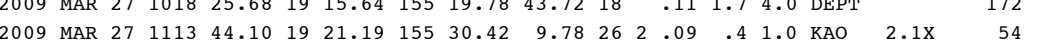

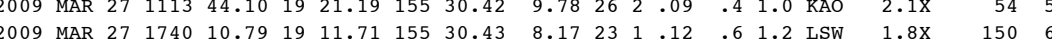

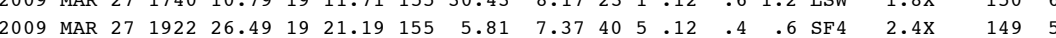
$\begin{array}{lllllllllllllllll}2009 & \text { MAR } 28 & 0058 & 23.04 & 19 & 24.82 & 155 & 15.87 & 5.99 & 7 & 1 & .05 & 1.2 & 3.0 & \text { INTL } & 1.5 \mathrm{X} & 165\end{array}$

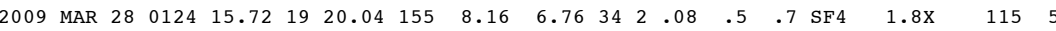

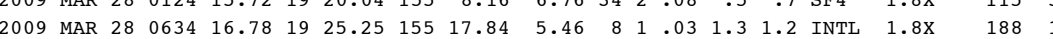

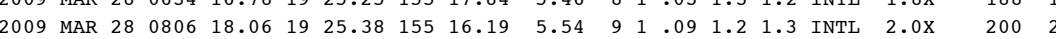

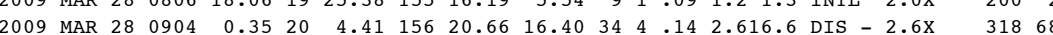

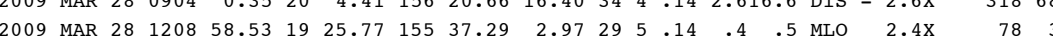

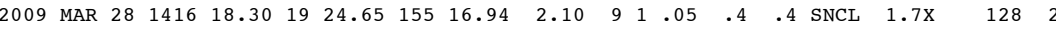

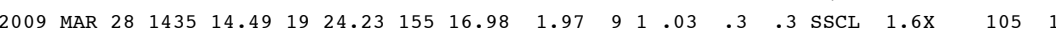

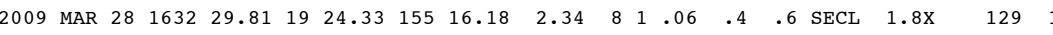

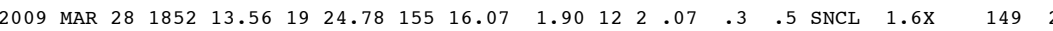

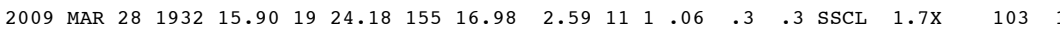

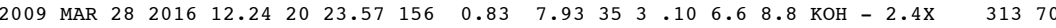

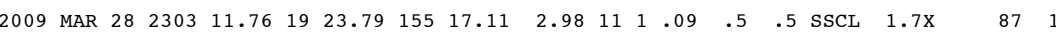

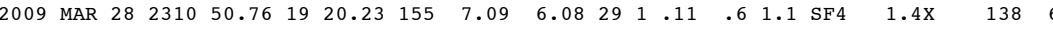

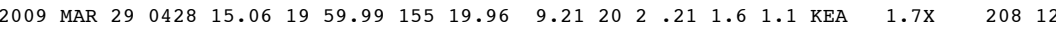

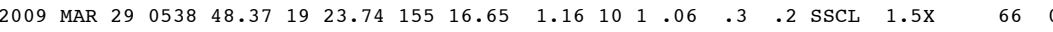

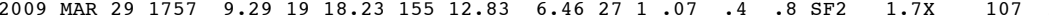

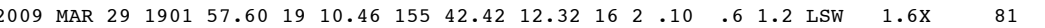

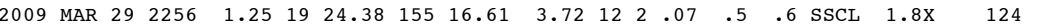

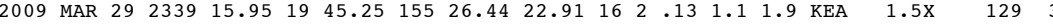

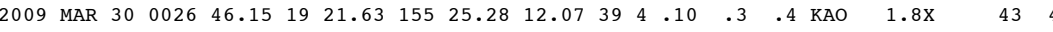

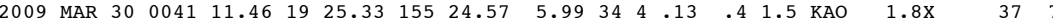

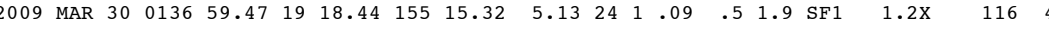

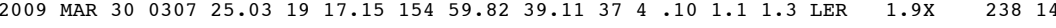

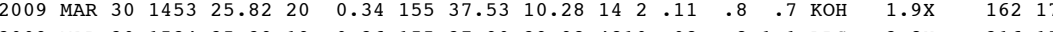

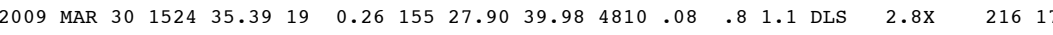

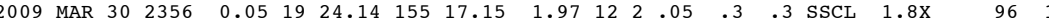

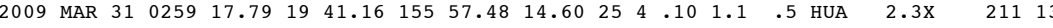

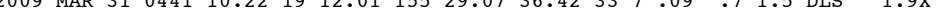

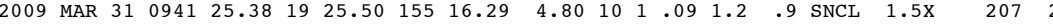

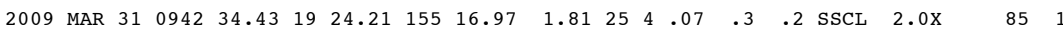

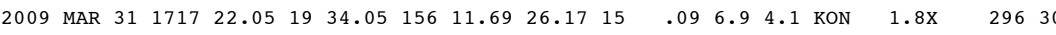


Table 5.

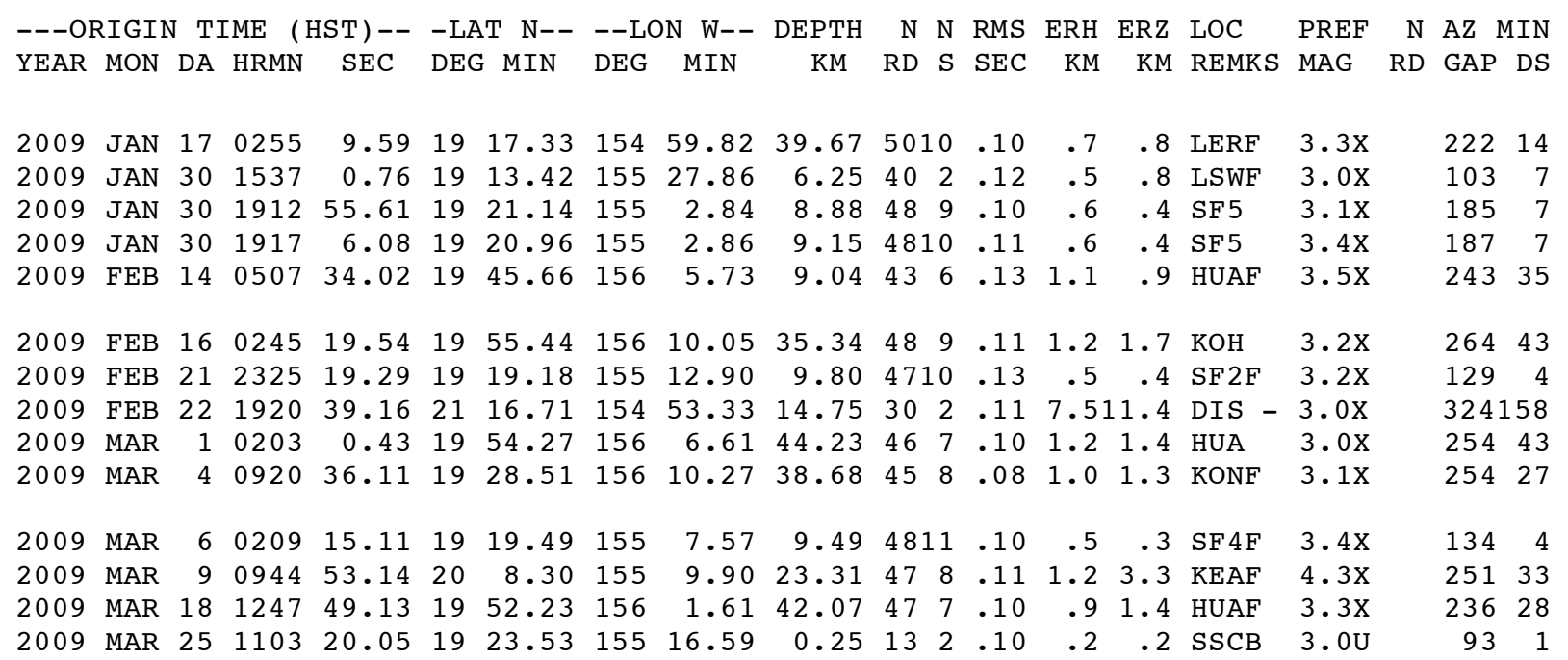



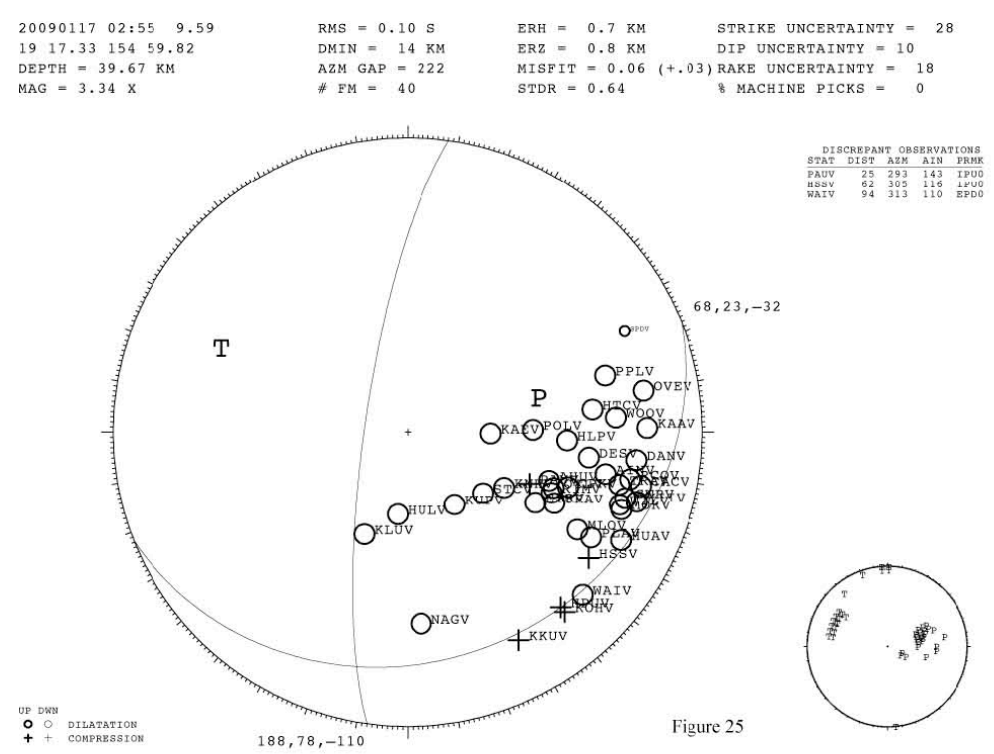

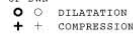

Figure 25

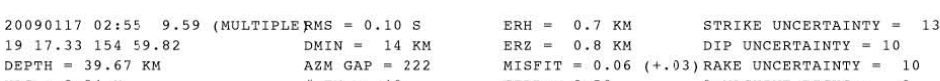

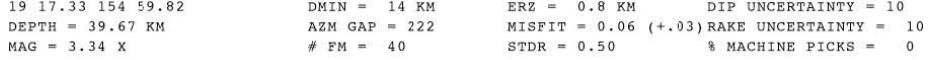

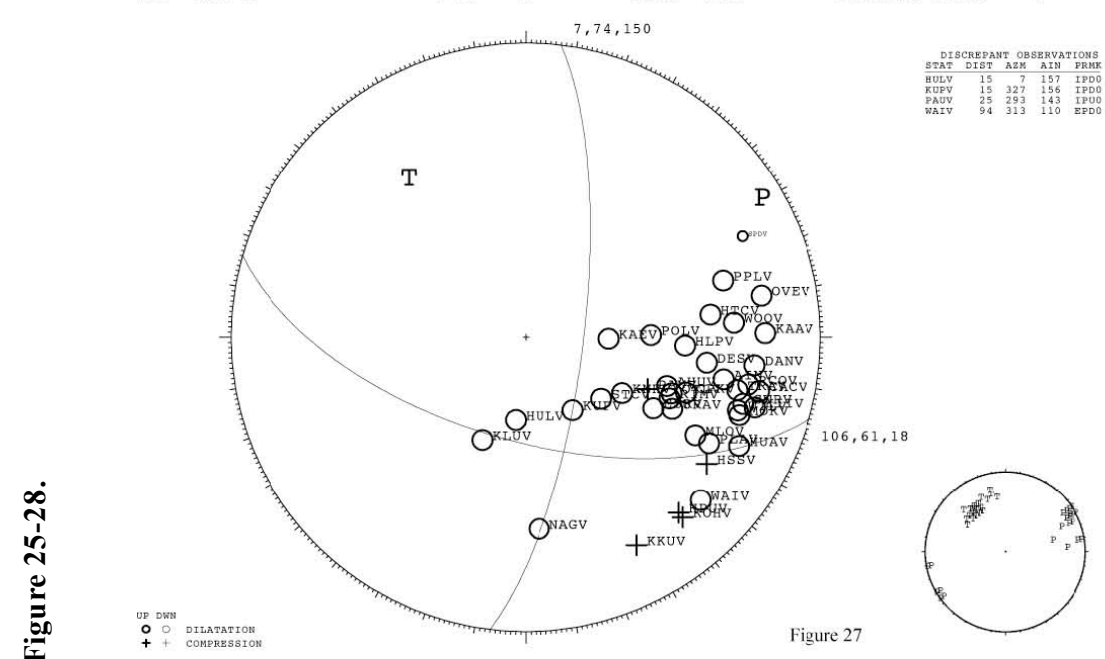

$2009011702: 559.59$ MULTIPLERMS $=0.10 \mathrm{~S} \quad$ ERH $=0.7 \mathrm{KM} \quad$ STRIKE UNCERTAINTY $=3$

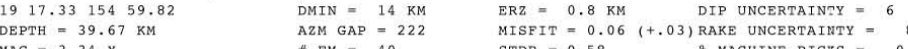
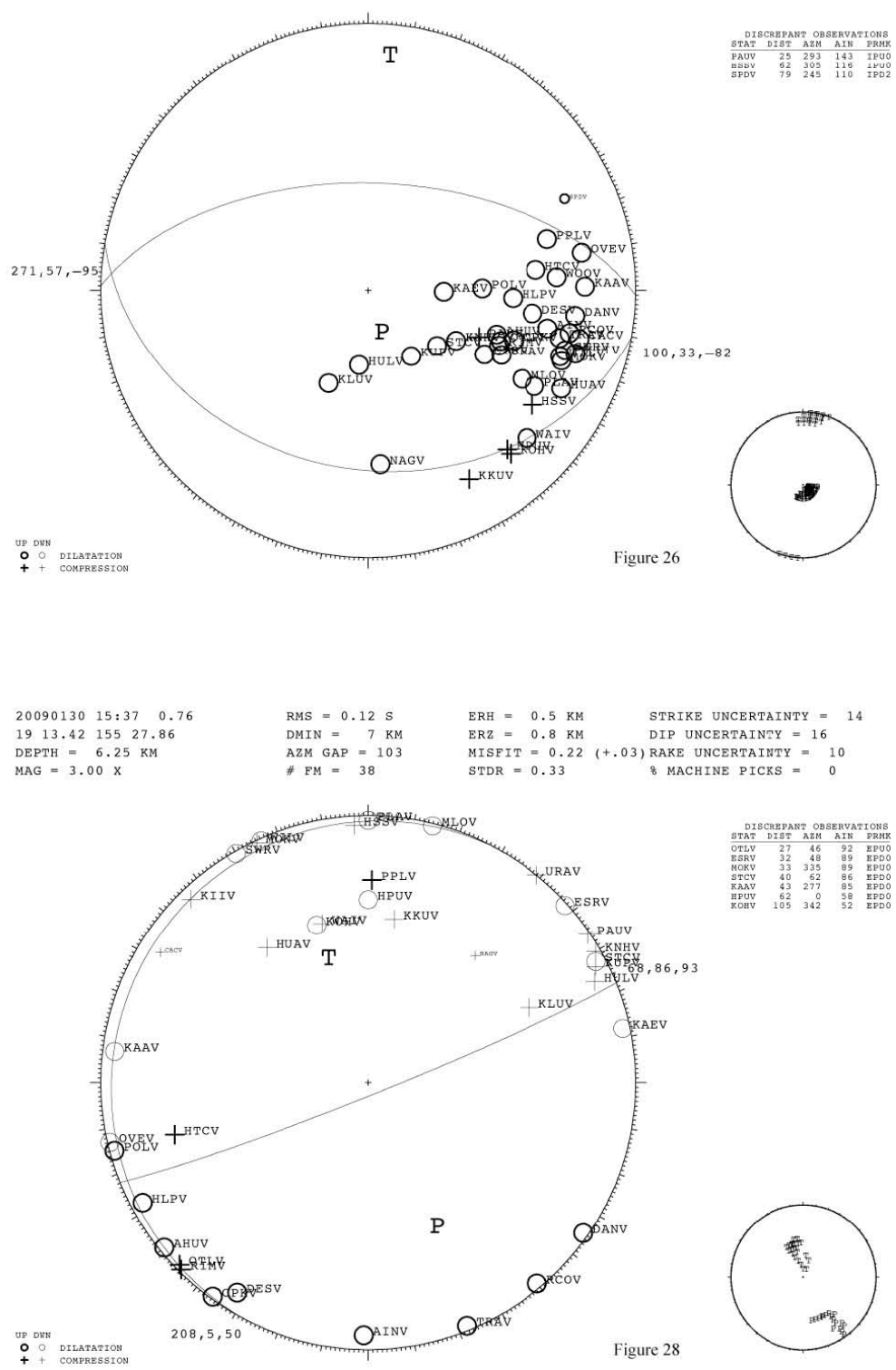

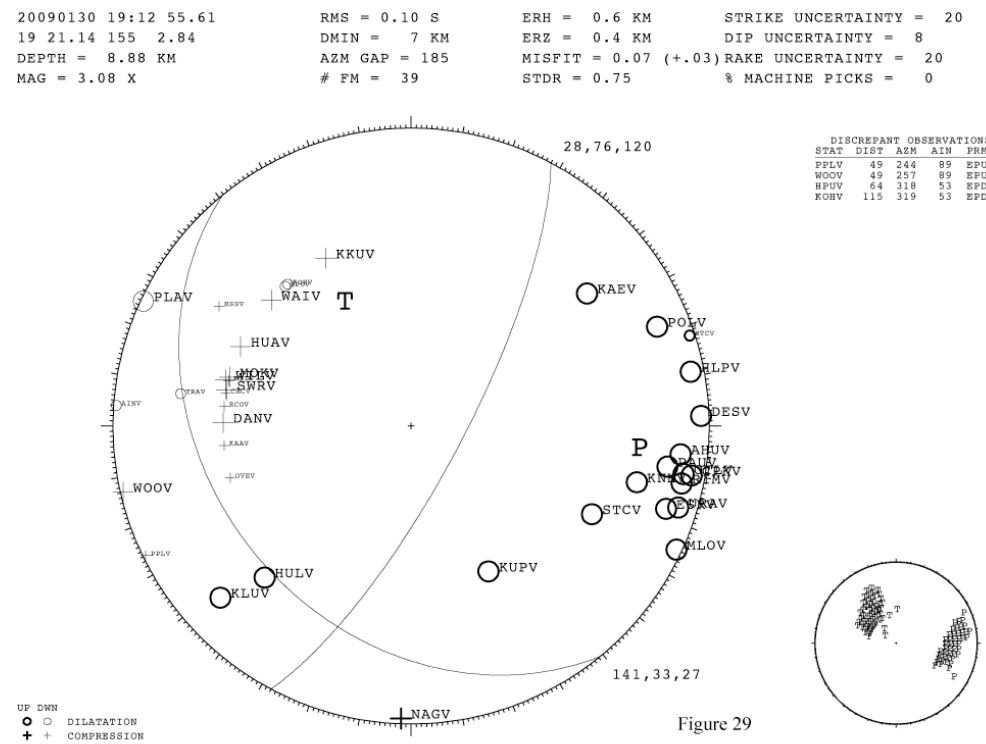

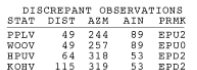

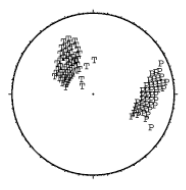

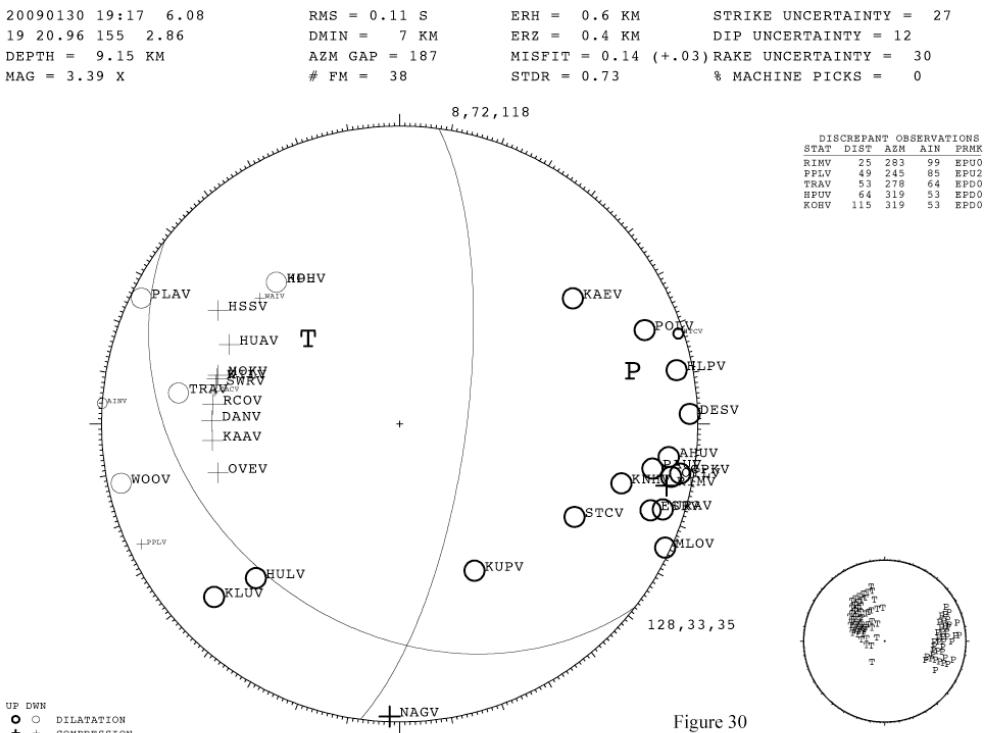

q
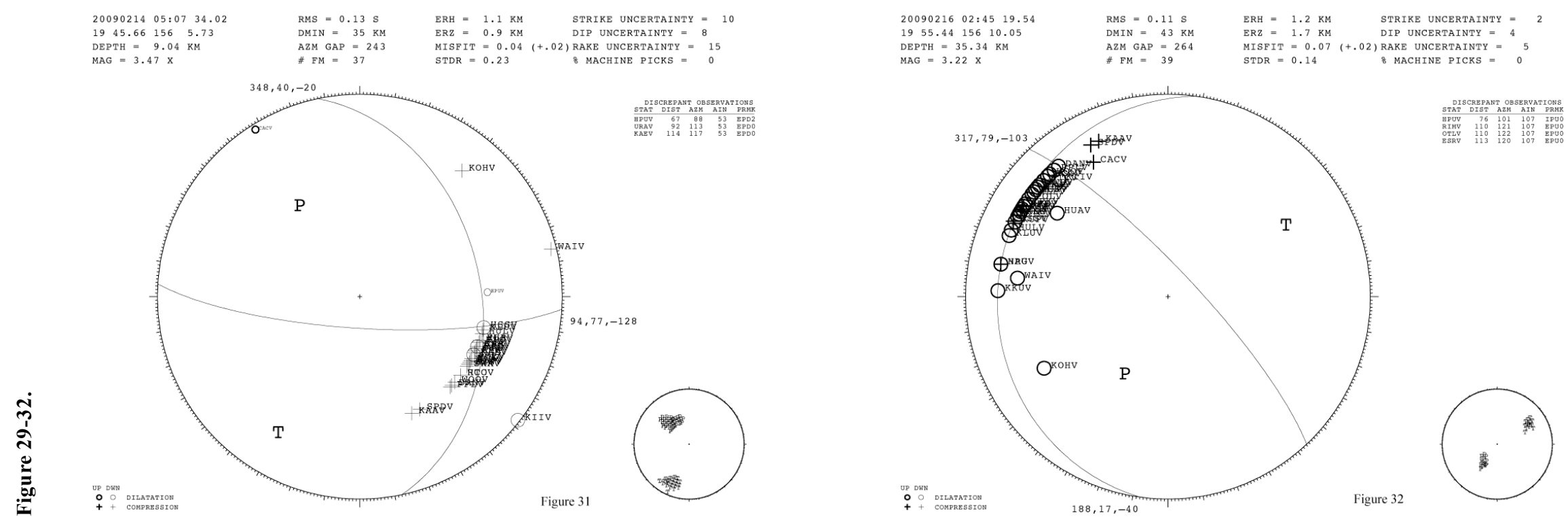

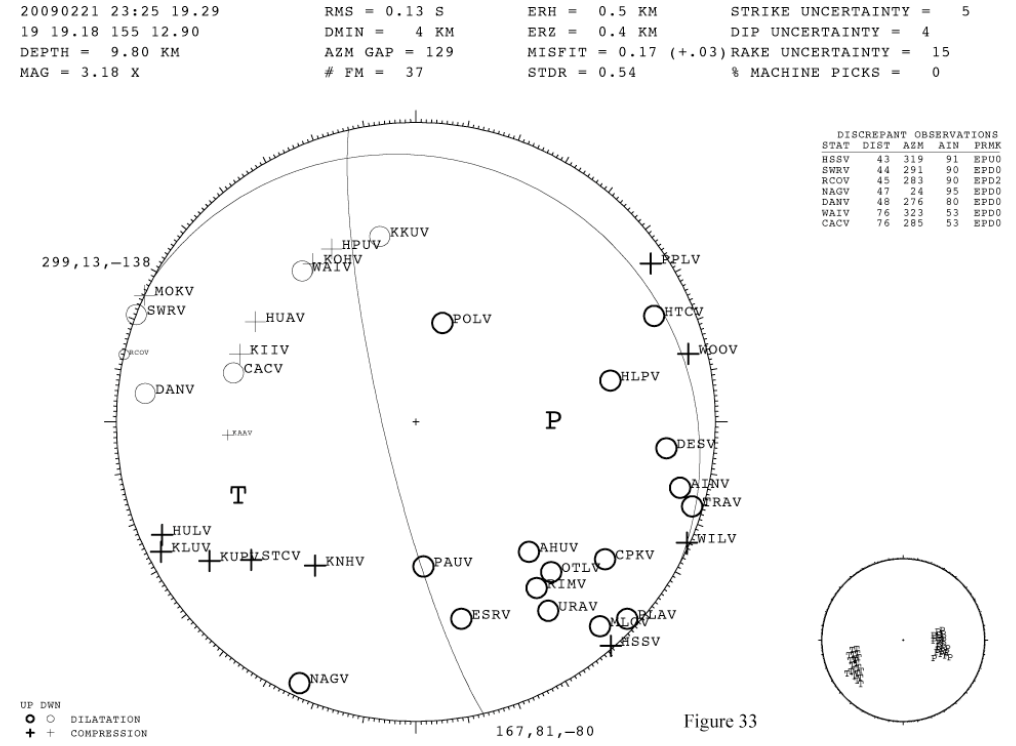

Figure 33

RH $=1.2 \mathrm{KM}$ STRIKE UNCERTAINTY $=$ $2009030102: 03 \quad 0$. $1954.27156 \quad 6$.
DEPTH $=44.23 \mathrm{KM}$
MAG $=3.03 \times 2$ RMS $=0.10 \mathrm{~s}$
DMIN $=43$ RM DMIN $=43$ RM
AZM GAP 254
H FM $=39$

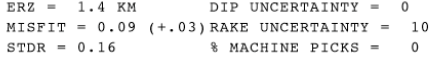

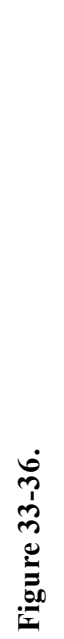
MAG $=3.03 \times$
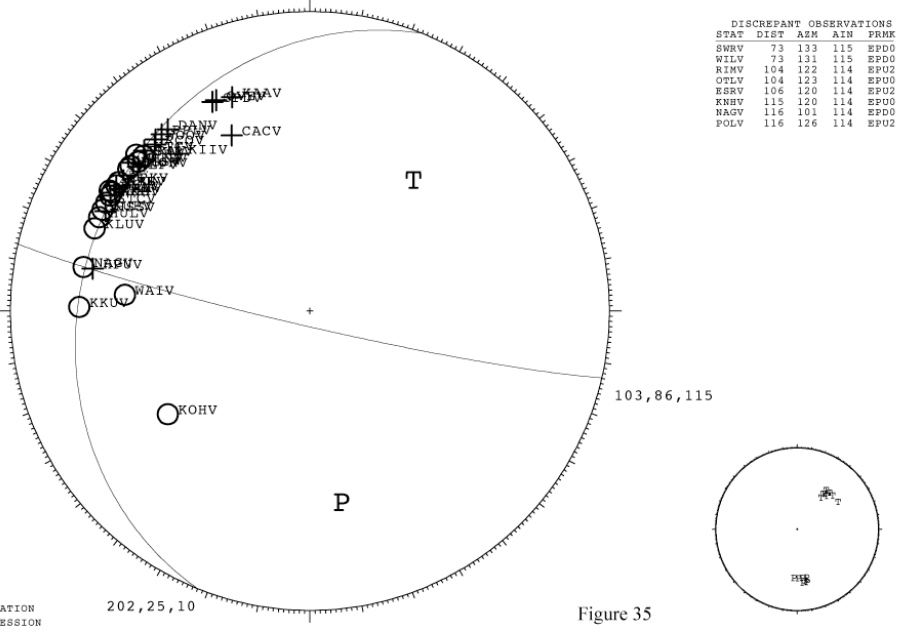

Figure 35

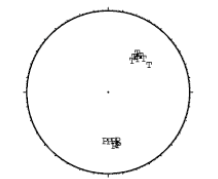

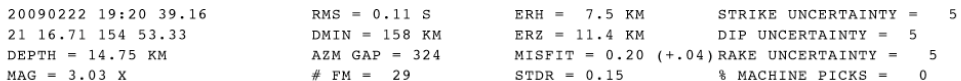

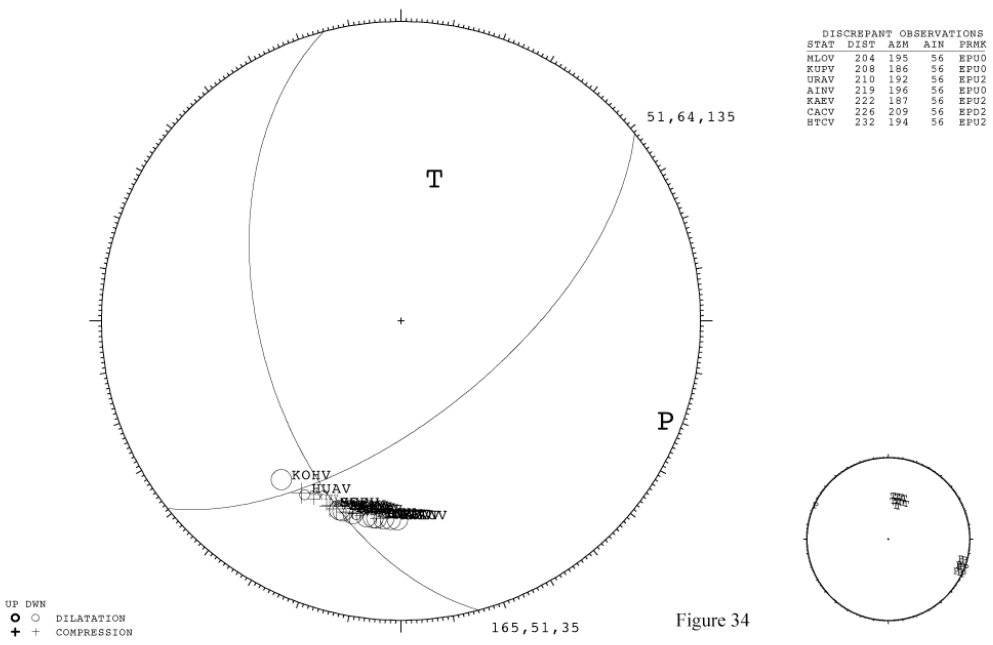




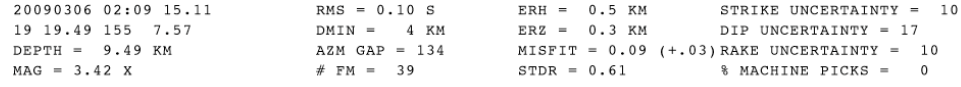

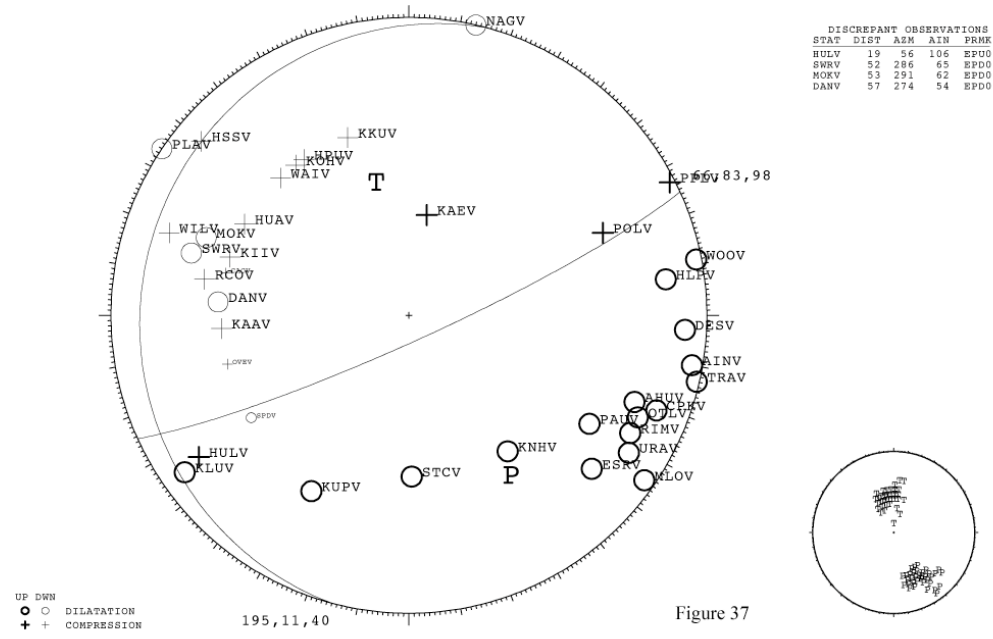

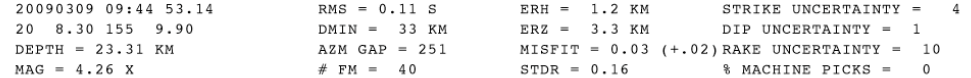

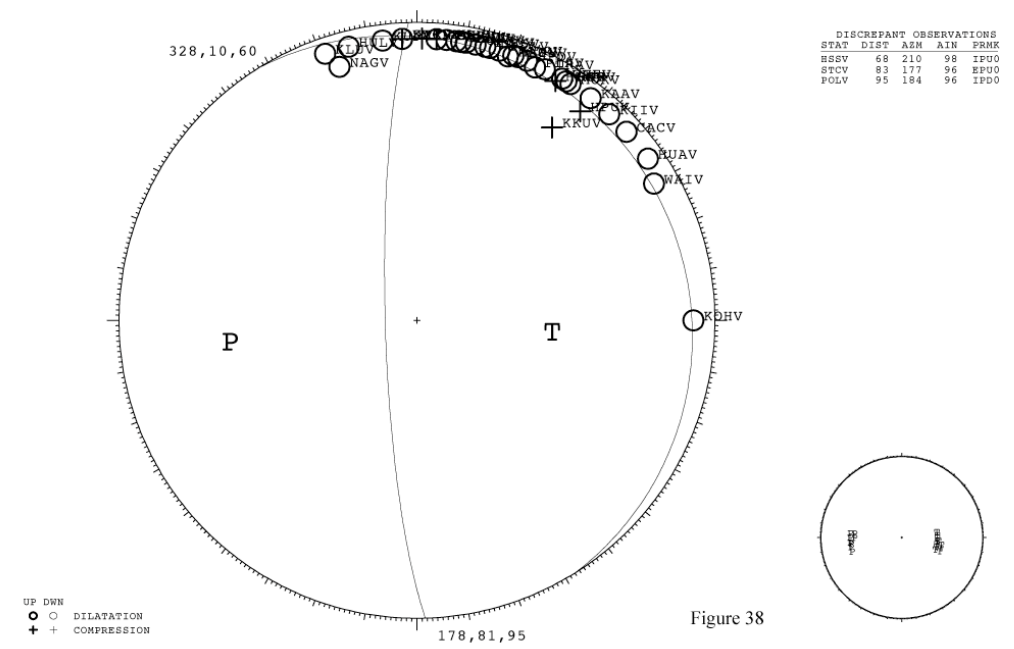

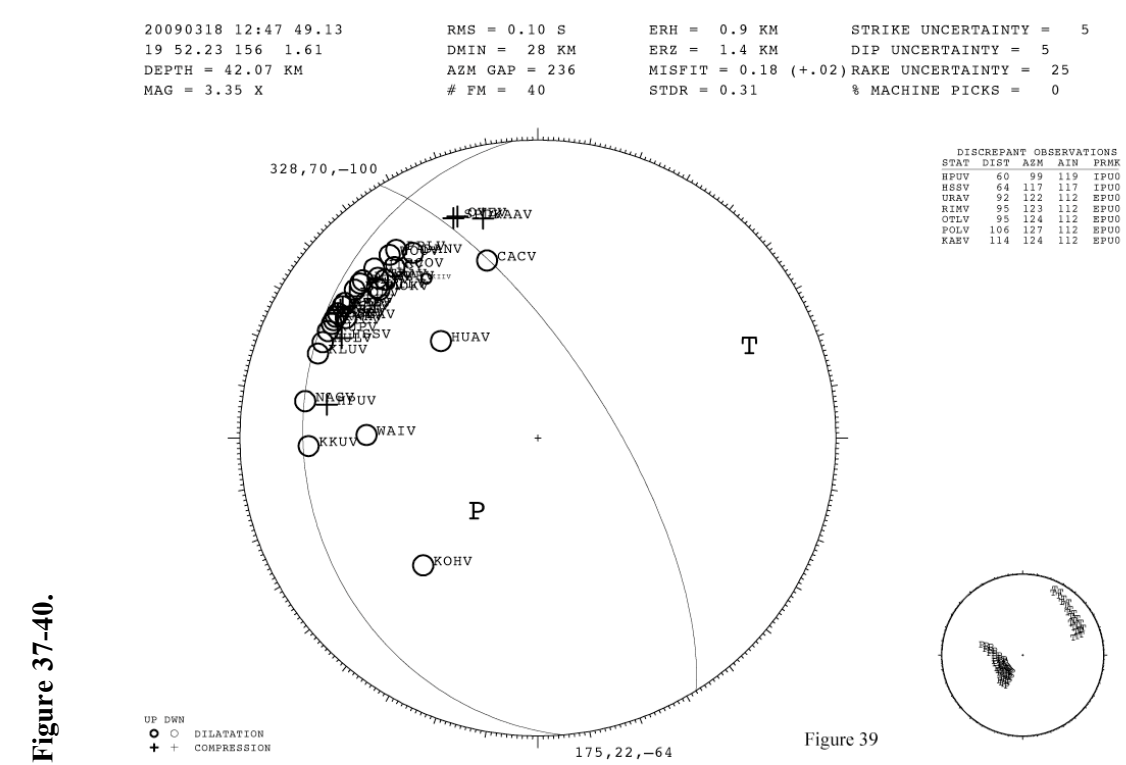

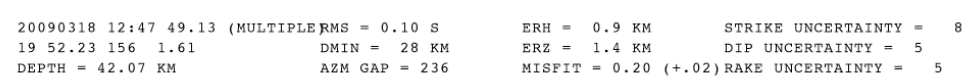

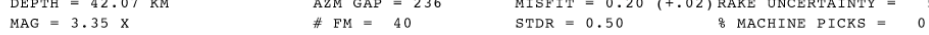

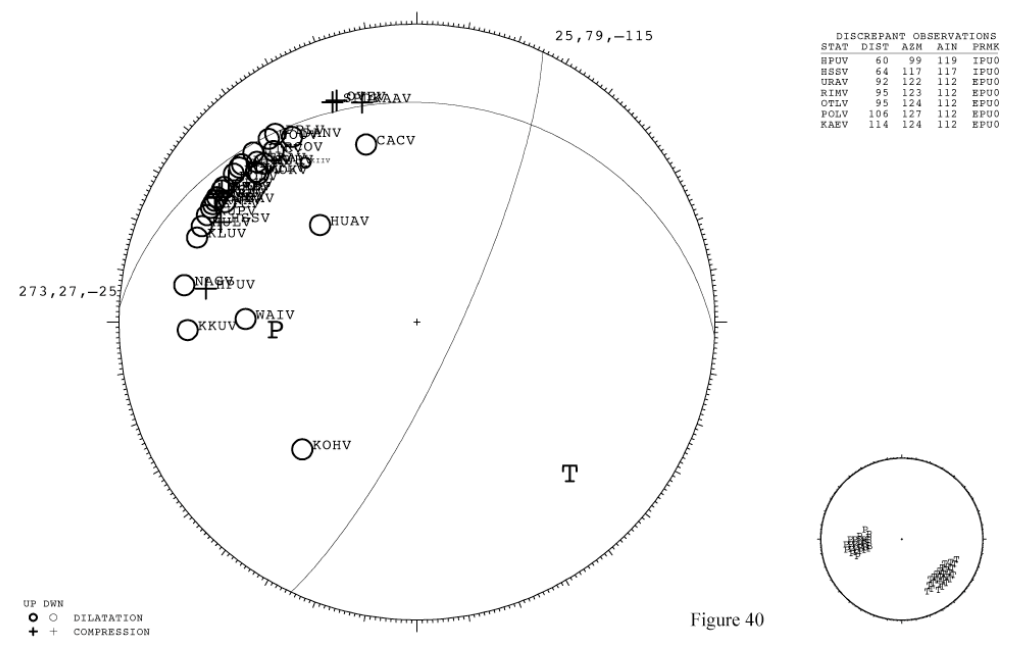

